\title{
An Inducible Cre Mouse with Preferential Activity in Vascular Smooth Muscle Evades a Previously Lethal Intestinal Phenotype
}

Ganesh D. Warthi, ${ }^{1}$ Jessica L. Faulkner, ${ }^{2}$ Jaser Doja,,${ }^{1}$ Amr R. Ghanam,,${ }^{1}$ Pan Gao, ${ }^{1}$ Allison C. Yang, ${ }^{1}$ Orazio J. Slivano, ${ }^{1}$ Candee T. Barris, ${ }^{1}$ Taylor C. Kress, ${ }^{1}$ Scott D. Zawieja, ${ }^{3}$ Susan H. Griffin, ${ }^{1}$ Xiaoling Xie, ${ }^{4}$ Alan Ashworth, ${ }^{5}$ Christine K. Christie, ${ }^{6}$ William B. Bryant, ${ }^{1}$ Ajay Kumar, ${ }^{1}$ Michael J. Davis, ${ }^{3}$ Xiaochun Long, ${ }^{1}$ Lin Gan, ${ }^{4}$ Eric J. Belin de Chantemèle, ${ }^{1}$ Qing Lyu,${ }^{1 *}$ Joseph M. Miano ${ }^{1^{*}}$

${ }^{1}$ Vascular Biology Center and ${ }^{2}$ Department of Physiology, Medical College of Georgia at Augusta University, Augusta, Georgia 30912; ${ }^{3}$ Medical Pharmacology and Physiology, University of Missouri School of Medicine, Columbia, MO 65212; ${ }^{4}$ Department of Neuroscience and Regenerative Medicine, Medical College of Georgia at Augusta University, Augusta, Georgia 30912; ${ }^{5}$ Helen Diller Family Comprehensive Cancer Center, University of California San Francisco, San Francisco, CA, 94158; ${ }^{6}$ Aab Cardiovascular Research Institute, University of Rochester School of Medicine \& Dentistry, Rochester, New York, 14642

*Corresponding Authors: Joseph M. Miano, PhD Vascular Biology Center Medical College of Georgia at Augusta University 1460 Laney Walker Boulevard Augusta, Georgia 30912 imiano@augusta.edu 706-446-0166 (Phone) 706-721-9799 (FAX)

Qing Lyu, PhD Biomedical and Health Institute Chongqing Institute of Green and Intelligence Technology Chinese Academy of Sciences Chongqing 400714, China lvqing@cigit.ac.cn 


\begin{abstract}
All smooth muscle cell (SMC) restricted Cre mice recombine floxed alleles in vascular and visceral SMCs. We generated a new tamoxifen-inducible CreER ${ }^{T 2}$ mouse, Itga8-CreER ${ }^{T 2}$, and compared its activity to the widely used Myh11-CreER ${ }^{T 2}$ mouse. Both CreER $R^{T 2}$ mice showed similar activity in vascular SMCs; however, Itga8-CreER ${ }^{T 2}$ displayed limited activity in visceral SMC-containing tissues (e.g., intestine). Myh11-CreER ${ }^{T 2}$ (but not Itga8-CreER ${ }^{T 2}$ ) mice displayed high levels of CreER ${ }^{\mathrm{T} 2}$ protein, tamoxifen-independent activity, and an altered transcriptome. Whereas Myh11-CreER ${ }^{T 2}$-mediated knockout of Srf resulted in a lethal intestinal phenotype, loss of Srf with Itga8-CreER $R^{T 2}\left(\mathrm{Srftga8}^{\mathrm{tg}}\right)$ revealed viable mice with attenuated vascular SMC contractile gene expression, but no evidence of intestinal pathology. Male and female $S r^{f t g a 8}$ mice presented with vascular contractile incompetence; however, only male Siftgas mice showed systemic changes in blood pressure. These results establish the Itga8-CreER ${ }^{T 2}$ mouse as an alternative to existing SMC Cre strains, including Myh11-CreER ${ }^{T 2}$, where SMC gene loss results in visceral myopathies that obfuscate accurate phenotyping in vascular SMCs.
\end{abstract}

Running title: New VSMC Cre driver mouse

Key Words: mouse; knockout; smooth muscle; serum response factor; Cre; integrin alpha 8 


\section{Introduction}

Conditional inactivation of a gene requires two components derived from bacteriophage P1: a Cyclization recombination (Cre) gene encoding a 343 amino acid ( 38 kDa) tyrosine sitespecific recombinase and 34-base pair locus of crossing ( $\underline{x})$ over of $\underline{P} 1$ (loxP) sequences having dyad symmetry, flanking (or floxing) the region of DNA to be excised. ${ }^{1,2}$ The first application of the Cre/loxP system for conditional knockout of a gene in mice utilized an Lck promoter-driven Cre transgene to delete the 5' promoter and first exon of a floxed Polb gene in T cells. ${ }^{3}$ This pioneering study underscores two critical issues in the design of conditional knockout mouse experiments, namely judicious placement of loxP sites around a sequence to be excised and restricted spatio-temporal expression of Cre. ${ }^{4}$ The spatial control of Cre expression is of utmost significance for the inactivation of genes in smooth muscle cells (SMCs) which are pervasive across the body plan, investing blood vessels and all hollow organs of the abdominal cavity.

SMCs have distinct embryological origins ${ }^{5,6}$ and contractile properties ${ }^{7-9}$, but they express a common set of cell-restricted genes that maintain normal SMC homeostasis. ${ }^{10}$ Several of these genes have been harnessed for the development of SMC Cre driver mice, each of which has advantages and disadvantages. ${ }^{11}$ A shared characteristic of all current SMC Cre driver mice is the recombination activity in both vascular and visceral SMC lineages. ${ }^{11}$ SMC-wide Cre activity can lead to severe, often lethal, visceral myopathies ${ }^{12-17}$ that may impede efforts to understand the biology of key genes within vascular SMCs (VSMCs). Moreover, whole body knockout of SMC-restricted genes each resulted in embryonic or postnatal death. ${ }^{18-}$ 21 Various gene editing strategies will accelerate new floxed genes for conditional knockout studies in $\mathrm{SMCs}^{22-25}$, and it is likely many gene products will be of vital importance for normal visceral SMC homeostasis. Clearly, there will be instances where a more restrictive VSMC Cre driver mouse will be necessary to circumvent the confounding effects of visceral myopathies.

Alpha 8 integrin (Itga8) was first cloned from an embryonic chicken cDNA library and the $\sim 160 \mathrm{kDa}$ protein product was shown to interact with the beta 1 integrin subunit. ${ }^{26}$ Independent studies reported ITGA8 protein in VSMCs, intestinal SMCs, and mesangial cells (a SMC-like cell) of the adult rat kidney. ${ }^{27,28}$ A screen for retinoid-inducible genes in VSMCs reported a partial cDNA to rat Itga8 that was used to show prominent Itga8 mRNA expression in the adult rat aorta, with no detectable signal in a panel of other adult tissues. ${ }^{29}$ Subsequent studies demonstrated the down-regulation of ITGA8 with vascular injury ${ }^{30}$ and its role in the 
maintenance of VSMC differentiation. ${ }^{31}$ Interestingly, the Myocardin (MYOCD) coactivator ${ }^{32}$ stimulated Itga8 expression in a serum response factor (SRF)-independent manner. ${ }^{33}$ The latter finding distinguishes Itga8 from most other SMC markers that have functional SRF-binding sites in the promoter or first intron. ${ }^{34}$

A previous report found Itga8 mRNA to be expressed abundantly in VSMCs with little to no detectable expression in most visceral SMC-containing tissues of mouse, rat, and human origin. This restricted pattern of expression in VSMCs across multiple species prompted the suggestion that the Itga8 gene might represent an ideal locus for targeting with an inducible Cre in order to establish a new mouse model for more restrictive conditional knockout studies in VSMCs. ${ }^{33}$ Here, the generation and characterization of an Itga8-CreER $R^{T 2}$ mouse is reported. Comparative studies indicate important advantages of the Itga8-CreER ${ }^{T 2}$ mouse over all other SMC Cre drivers for the unequivocal determination of gene function in VSMCs. 


\section{Results}

\section{Itga8-CreER ${ }^{T 2}$ mice express normal levels of ITGA8 protein and a nuclear LncRNA}

Among known SMC markers, the Itga8 gene has the distinction of being expressed preferentially in VSMCs (Supplementary Fig. 1). We therefore targeted the first exon of Itga8 with a CreER ${ }^{T 2}$ cassette $^{35}$ in embryonic stem cells to establish a new inducible Cre driver mouse (Supplementary Fig. 2). Mice homozygous null for Itga8 display renal agenesis and postnatal death (Supplementary Fig. 3). ${ }^{36}$ Because ITGA8 protein expression was not reported in heterozygous knockout mice, ${ }^{36}$ it was important to determine whether one functional Itga8 allele is sufficient for normal levels of ITGA8 protein expression. Results showed no change in ITGA8 protein, but a small reduction of Itga8 mRNA in the aorta of heterozygous Itga8-CreER ${ }^{T 2}$ mice (Supplementary Fig. 4). Further, there was little change in expression of a 5' overlapping nuclear long non-coding RNA (Supplementary Fig. 4). Male and female Itga8-CreER ${ }^{T 2}$ mice displayed similar levels of ITGA8 and CreER ${ }^{\top 2}$ protein in aorta (Supplementary Figure 4) and they bred well with no overt signs of pathology. These results suggest a compensatory increase in ITGA8 expression from the remaining functional Itga8 allele, supporting accurate phenotyping of conditional VSMC knockout phenotypes without the potential confounding effects of reduced ITGA8 protein.

\section{Itga8-CreER ${ }^{T 2}$ mice display preferential activity in VSMCs}

More than 300 floxed genes have been targeted with various SMC Cre driver mice, 90\% of which were generated with several versions of Sm22-Cre or Myh11-Cre (Supplementary Table 1). Previous work with Tagln-Cre (aka Sm22-Cre) reported recombination in myeloid cell lineages. ${ }^{37}$ To further address myeloid cell activity in SMC Cre mice, circulating cells from male and female Itga8-CreER ${ }^{T 2}$ mice carrying the $\mathrm{mT} / \mathrm{mG}$ reporter were isolated and compared to similarly prepared cells from male Sm22-Cre ${ }^{38}$ and Myh11-CreER ${ }^{T 239}$ mice. As expected, fluorescence-activated cell sorting revealed $\sim 22 \%$ GFP positive cells in Sm22-Cre mice; however, no GFP fluorescence was observed in circulating cells of Itga8-CreER ${ }^{T 2}$ or Myh11CreER ${ }^{T 2}$ mice suggesting the absence of CreER ${ }^{T 2}$ activity in myeloid cells of these two SMC Cre drivers (Supplementary Fig. 5). Given the broader activity of Sm22-Cre, ${ }^{37,38}$ the balance of experiments were conducted only in Itga8-CreER $R^{T 2}$ and Myh11-CreER $R^{T 2}$ mice. 
Confocal immunofluorescence microscopy was used to compare the activity of Itga8CreER ${ }^{T 2}$ with Myh11-CreER ${ }^{T 2}$ across multiple tissues from age-matched mice carrying the same $\mathrm{mT} / \mathrm{mG}$ reporter used in the above flow studies. The absence of CreER ${ }^{\mathrm{T} 2}$ activity is indicated by red (tomato) fluorescence whereas CreER ${ }^{\mathrm{T} 2}$-mediated excision of a stop floxed cassette unveils green (GFP) fluorescence. Both Itga8-CreER ${ }^{T 2}$ and Myh11-CreER ${ }^{T 2}$ showed activity in SMCs of the aorta, mesentery, and microvasculature of brain and visceral organs such as intestine and bladder (Fig. 1). Higher magnification, z-stacked confocal images revealed obvious GFP fluorescence in SMCs of the aorta and vena cava with no observable GFP in endothelial cells or adventitial cells (Supplementary Fig. 6). Myh11-CreER ${ }^{T 2}$ showed high activity in visceral SMCs of bladder, colon, esophagus, intestine, stomach, and ureter (Fig. 1). In contrast, male and female Itga8-CreER ${ }^{T 2}$ mice displayed lower activity in each of these visceral SMC-enriched tissues (Fig. 1). Low Itga8-CreER ${ }^{T 2}$ activity was also seen in uterus (Fig. 1); no comparison could be made in uterus with Myh11-CreER ${ }^{T 2}$ because this transgene integrated on the $Y$ chromosome (see below). ${ }^{39}$ There was little difference in activity between the two Cre drivers in tracheal and bronchiolar SMCs of the respiratory system (Fig. 1). Neither Itga8-CreER ${ }^{T 2}$ nor Myh11-CreER ${ }^{T 2}$ showed detectable activity (outside of blood vessels) in tissues of the eye, heart, liver, pancreas, skeletal muscle, and spleen as well as germ cells of the testis and ovary (Supplementary Fig. 6). However, Myh11-CreER ${ }^{T 2}$ mice exhibited pronounced activity in cells of the thymus and Itga8-CreER $R^{T 2}$ mice disclosed activity in glomerular cells of the kidney (Supplementary Fig. 6). These results demonstrate some similarities, but important differences, in the activity of Myh11-CreER ${ }^{T 2}$ and Itga8-CreERT2.

\section{Myh11-CreER ${ }^{T 2}$ mice exhibit leaky activity and an altered basal transcriptome}

There is evidence for tamoxifen-independent (leaky) recombination events with the CreER ${ }^{T 2}$ cassette. ${ }^{40}$ Accordingly, individually-housed male Itga8-CreER ${ }^{T 2}$ or Myh11-CreER ${ }^{T 2}$ mice carrying the $m T / m G$ reporter were analyzed for unscheduled recombination. Whereas no detectable recombination of the $m T / m G$ reporter was evident in Itga8-CreER ${ }^{T 2}$ mice, clear evidence of tamoxifen-independent recombination (ie, GFP fluorescence) was apparent in multiple tissues of Myh11-CreER ${ }^{T 2}$ mice (Fig. 2a). To begin to explore the basis for such leaky activity, levels of CreER ${ }^{\mathrm{T} 2}$ protein were analyzed in the aorta and bladder of age-matched male mice. Western blotting showed much higher $\mathrm{CreER}^{\mathrm{T} 2}$ protein expression in the aorta and 
bladder of Myh11-CreER ${ }^{T 2}$ mice than Itga8-CreER ${ }^{T 2}$ mice (Fig. 2b). Sanger sequencing disclosed the Cre in Myh11-CreER ${ }^{T 2}$ to be codon optimized for more efficient translation of the bacterial Cre protein in mammals (Supplementary Fig. 7). ${ }^{41}$ Further, quantitative PCR of genomic DNA revealed two copies of the Myh11-CreER ${ }^{T 2}$ transgene had integrated in the genome (Fig. 2c). Notably, long read sequencing demonstrated $X$ chromosome-specific sequence near the pseudoautosomal region (PAR), flanking the integrated Myh11-CreER ${ }^{T 2}$ transgenes, suggesting an initial integration on the $X$ chromosome followed by translocation to the $Y$ chromosome (Fig. 2d). Bulk RNA-seq studies disclosed elevated expression of 101 genes in the aorta of Myh11-CreER ${ }^{T 2}$ mice as compared to wild type and Itga8-CreER ${ }^{T 2}$ mice (Fig. 2e), including two X-linked genes (Arhgap6 and Hccs) found in close proximity to the Myh11-CreER ${ }^{T 2}$ transgenes (Fig. 2d, 2e). Collectively, these results demonstrate high level CreER ${ }^{\mathrm{T} 2}$ protein expression and an unusual chromosomal translocation event in Myh11CreER ${ }^{T 2}$ mice, each of which appears to contribute to tamoxifen-independent activity and an altered baseline transcriptome.

\section{Recombination efficiency of Itga8-CreER ${ }^{T 2}$ and Myh11-CreER ${ }^{T 2}$}

The differential expression of CreER ${ }^{\top 2}$ protein suggested there could be differences in recombination efficiency between the two CreER ${ }^{T 2}$ driver strains. Several independent floxed mouse models were tested for recombination efficiency between Myh11-CreER ${ }^{T 2}$ and Itga8CreER ${ }^{T 2}$ mice. Quantitative PCR of genomic DNA before and after tamoxifen administration showed comparable recombination of the $m T / m G$ reporter in aorta of both CreER ${ }^{T 2}$ strains (Fig. $3 a, 3 b)$. As expected, there was high-level recombination in bladder of Myh11-CreER ${ }^{T 2}$ mice, but very little in the bladder of Itga8-CreER ${ }^{T 2}$ mice (Fig. 3a, 3b). Similar findings were observed in a tamoxifen-inducible Myocardin mouse (Fig. 3c, 3d). Next, the activity of each CreER ${ }^{T 2}$ strain was quantitated in popliteal vessels. Results showed $\sim 90 \%$ and $\sim 65 \%$ recombination efficiency in lymphatic vessels of the $\mathrm{mT} / \mathrm{mG}$ reporter in Myh11-CreER ${ }^{T 2}$ and Itga8-CreER ${ }^{T 2}$ mice, respectively (Fig. 3e, 3f). Recombination efficiencies were somewhat lower in popliteal artery and vein, but the Myh11-CreER ${ }^{T 2}$ activity was higher than Itga8-CreER ${ }^{T 2}$ (Supplementary Fig. 8). Finally, each CreER ${ }^{T 2}$ strain was crossed to a floxed Srf mouse ${ }^{38}$ treated with oil or tamoxifen. Normalized SRF protein was similarly knocked down in the aorta of each CreER ${ }^{T 2}$ strain; however, only Myh11-CreER ${ }^{T 2}$ showed significant knockdown of SRF in bladder (Fig. 3g, 
3h). Overall, despite much lower expression of $\mathrm{CreER}^{\mathrm{T} 2}$ protein in Itga8-CreER ${ }^{T 2}$ mice, excision of floxed sequences approximates that seen in Myh11-CreER ${ }^{T 2}$ mice, though there appear to be context-dependent variances in recombination efficiency with different floxed alleles.

\section{Itga8-CreER ${ }^{T 2}$ circumvents a previously lethal visceral myopathy upon knockdown of Srf}

Srf loss-of-function studies using an Sm22-CreER ${ }^{T 2}$ driver mouse demonstrated a lethal gastrointestinal phenotype. ${ }^{12,13}$ Consistent with these findings, homozygous floxed Srf mice carrying Myh11-CreER ${ }^{T 2}$ displayed uniform lethality within 3-4 weeks of tamoxifen administration. Mutant mice showed a pale, distended intestine and a pronounced reduction in SRF expression within the muscular layer of the small intestine (Supplementary Fig. 9). In contrast, floxed Srf mice carrying the Itga8-CreER ${ }^{T 2}$ driver were viable with no intestinal phenotype up to 8 weeks post-tamoxifen administration (Supplementary Fig. 9 and below). Confocal immunofluorescence microscopy demonstrated a clear reduction of SRF and ACTA2 in carotid artery of Itga8-CreER ${ }^{T 2}$-mediated Srf knockout mice, but not in intestinal SMCs (Supplementary Fig. 10). Quantitative measures of the change in SRF protein on sections of carotid artery and intestine were consistent with confocal images (Supplementary Fig. 10). Additional Western blotting indicated a reduction in SRF of homozygous floxed (HoF) Srf knockout mice, but no significant change in bladder of the same mice (Supplementary Fig. 10). Notably, there was little change in SRF expression in aorta of heterozygous floxed (HeF) Srf knockout mice, suggesting compensatory expression from the remaining wild type allele (Supplementary Fig. 10). Because of the activity of Itga8-CreER ${ }^{T 2}$ in the glomerulus (Fig. 1), we considered the possibility of a kidney phenotype with conditional inactivation of Srf.

Homozygous and heterozygous conditional Srf knockout mice displayed similar body weights and a normal histological appearance of kidneys, with no evidence of proteinuria (Supplementary Fig. 11). Taken together, these results establish the utility of the Itga8-CreER ${ }^{T 2}$ driver in evading an otherwise lethal, visceral myopathy upon inducible inactivation of Srf in Myh11-CreER ${ }^{T 2}$ mice.

\section{Itga8-CreER ${ }^{T 2}$-mediated Srf knockout causes defective vascular contractile activity}

To further demonstrate the utility of Itga8-CreER ${ }^{T 2}$, blood pressure and vascular contractile competence were assessed in male and female mice following selective loss of Srf. 
Telemetry measures in male mice demonstrated no change in baseline blood pressure. However, following angiotensin II infusion, significant increases in systolic, diastolic, and mean arterial pressures were observed in control mice, but not in conditional Srf knockout mice (Fig. 4). Interestingly, age-matched female mice failed to respond to angiotensin II and showed no changes in blood pressure, regardless of Srf status (Fig. 5). On the other hand, the aorta of male and female mice displayed attenuated contraction in response to $\mathrm{KCl}$ (Fig. 6a) and phenylephrine (Fig. 6b, 6c). Immunofluorescence confocal microscopy revealed a clear decrease in SRF expression within SMCs of the aorta (Fig. 6d). Finally, bulk RNA-seq studies of the aorta from conditional Srf knockout mice yielded significant reduction in expression of several SMC contractile genes (Fig. 7a, 7b). Analysis of the top 250 down-regulated genes disclosed the SRF binding CArG box as the most significantly enriched transcription factor binding site (Fig. 7c). Further assessment of the down-regulated gene set revealed molecular functions related to the cytoskeleton and contractile machinery (Fig. 7d). Interestingly, the upregulated gene set following conditional Srf knockout was most related to metabolism (Fig. $7 e$ ). Collectively, these results demonstrate an unambiguous, viable mouse model of VSMC contractile incompetence following Itga8-CreER ${ }^{T 2}$-mediated inactivation of Srf. 


\section{Discussion}

All currently available SMC Cre mice, including the popular Myh11-CreER ${ }^{T 2}$ mouse, ${ }^{39}$ direct excision of floxed DNA in both vascular and visceral SMC lineages. Consequently, the inactivation of a critical gene in, for example, SMCs of the colon may result in a lethal phenotype and the inability to accurately interrogate gene function in VSMCs. Here, we show preferential activity of Itga8-CreER ${ }^{T 2}$ in SMCs of the vascular and lymphatic system with comparatively less activity in visceral SMCs of the gastrointestinal (e.g., stomach and intestine) and urogenital (e.g., bladder and uterus) systems. Despite low level expression of CreERT2 in Itga8-CreER ${ }^{T 2}$ mice, recombination of several floxed sequences occurs with no evidence of leaky activity. In contrast, Myh11-CreER ${ }^{T 2}$ mouse tissues show much higher levels of CreERT2 expression due to two copies of the transgene, improved codon usage for Cre and, perhaps, higher promoter activity, all of which likely promote the observed leaky activity of this SMC Cre driver mouse. We also demonstrate an altered transcriptome in Myh11-CreER ${ }^{T 2}$ mice, including elevated transcripts of two $X$ chromosome-linked genes that we demonstrate with long read sequencing are contiguous with the BAC-containing Myh11-CreER ${ }^{T 2}$ transgenes (see below). Despite several distinctions between Myh11-CreER ${ }^{T 2}$ and Itga8-CreER ${ }^{T 2}$ (Table), the Myh11-CreER $R^{T 2}$ driver has been used successfully to inactivate well over 100 floxed alleles in SMCs (Supplementary Table 1).

However, a clear advantage of Itga8-CreER ${ }^{T 2}$ over Myh11-CreER ${ }^{T 2}$ is demonstrated here for the conditional inactivation of Srf in adult mice. The latter Cre driver resulted in a lethal intestinal phenotype within 3-4 weeks of tamoxifen administration. These findings are consistent with previous reports showing comparable intestinal pathology and early demise using Tagln-CreER ${ }^{T 2} 12,13$ and the aforementioned Myh11-CreER ${ }^{T 2}{ }^{16}$ On the other hand, mice with the same homozygous floxed Srf and a similar schedule of tamoxifen administration to induce Itga8-CreER $R^{T 2}$-mediated recombination, were viable for at least 8 weeks post-tamoxifen with no evidence of intestinal pathology. The simplest explanation for the absence of any overt intestinal phenotype is the much lower activity of Itga8-CreER ${ }^{T 2}$ in intestinal SMCs. This inference is based on results of the $m T / m G$ reporter studies and quantitative immunofluorescence microscopy experiments showing comparable levels of SRF protein in intestinal SMCs of mice with one or both alleles of Srf inactivated. 
The Itga8-CreER ${ }^{T 2}$ mouse opens the door to new experiments in VSMCs that were previously problematic because of confounding effects of gene loss in visceral SMC tissues such as intestine and bladder. In this context, although short-term (14 day) studies of conditional loss of Srf in VSMCs with Sm22-CreER ${ }^{T 2}$ have been reported, yielding insight into mechanosensitivity and blood flow ${ }^{42}$ as well as arterial stiffness, ${ }^{43}$ phenotyping occurred at a time when there is dilation of intestine, defective peristalsis, cachexia, and even death. ${ }^{12,} 13$ These intestinal phenotypes would certainly elicit inflammation and the release of circulating chemokines that likely impact VSMC function. Future acute Srf loss-of-function studies in VSMCs can now be carried out without confounding effects of gastrointestinal pathologies. Further, the Itga8-CreER ${ }^{T 2}$ mouse will be of great value in assessing loss of VSMC Srf (and other critical genes; see Supplementary Table 1) in more chronic vascular injury models such as aneurysmal formation, atherosclerosis, transplant arteriopathy, and hypertension.

It should be noted that heterozygous Srf knockout mice are not haploinsufficient and exhibit both wild expression of SRF protein as well as normal tissue structure and function. This highlights an important consideration for controlling conditional knockout studies. If loss of one allele does not result in a reduction of protein expression, as seen here with SRF, then the most stringent control would be heterozygous floxed mice carrying Cre with the same tamoxifen schedule used in homozygous floxed mice. However, the inclusion of $\mathrm{Cre}$ and a heterozygous floxed allele may not always be the best control. For example, the Myh11-CreER ${ }^{T 2}$ strain studied here shows leaky CreER ${ }^{\top 2}$ activity indicating an overestimation in the number of SMCs that underwent tamoxifen-mediated excision. In addition, the altered transcriptome seen in Myh11-CreER ${ }^{T 2}$ mice could confound the interpretation of a phenotype. In both cases, a better control would be tamoxifen-treated Myh11-CreER ${ }^{T 2}$ mice carrying wild type alleles. We suggest careful analysis of floxed mice before embarking on conditional knockout studies to inform the best control model to use in experiments.

The molecular basis for leaky activity in the Myh11-CreER $R^{T 2}$ mouse is unknown. The higher levels of CreER ${ }^{\text {T2 }}$ protein in Myh11-CreER ${ }^{T 2}$ over Itga8-CreER ${ }^{T 2}$ mice would seem to be a contributing factor. It is possible high levels of CreER ${ }^{\mathrm{T} 2}$ protein favor dissociation from the chaperone, HSP90, which anchors CreER ${ }^{\mathrm{T} 2}$ in the cytoplasm thereby prohibiting entry into the nucleus. ${ }^{44}$ In addition, the Myh11-CreER ${ }^{T 2}$ mouse has been bred far longer, with an unknown generation number, than Itga8-CreER ${ }^{T 2}$ allowing for a greater mutational load in the genome 
and the potential for modifiers influencing the trafficking of CreER ${ }^{T 2}$. In this context, strict breeding practices should be followed with frequent refreshing of the wild type colony to prevent genetic drift and the emergence of a substrain of Cre driver. Whatever the mechanism(s) for a leaky Myh11-CreER ${ }^{T 2}$, it is advised to routinely evaluate any Cre driver mice with a reporter for determination of accurate activity and to avoid continuous sibling mating for purposes of maintaining the line.

Another advantage of the Itga8-CreER ${ }^{T 2}$ mouse over the Myh11-CreER ${ }^{T 2}$ mouse is the ability to perform sex studies which enables compliance with the National Institutes of Health directive of addressing sex as a biological variable in research projects. ${ }^{45}$ Of note, the low activity of Itga8-CreER ${ }^{T 2}$ in uterine SMCs suggests this CreER ${ }^{T 2}$ driver may not be useful for studying effects of conditional SMC knockout studies in the female reproductive track. Although the Myh11-CreER ${ }^{T 2}$ mouse carries the CreER ${ }^{T 2}$ allele on the $Y$ chromosome, thereby preventing studies in females, a prior report showed translocation of Myh11-CreER ${ }^{T 2}$ to the $\mathrm{X}$ chromosome and the generation of female mice that display Myh11-CreER ${ }^{T 2}$ activity. ${ }^{46}$ It should be pointed out, however, that the established $\mathrm{t}(\mathrm{Y} ; \mathrm{X})$ Myh11-CreER ${ }^{T 2}$ line was not characterized with respect to site-of-integration on the $X$ chromosome ${ }^{46}$ and no study has yet to report the insertion site of Myh11-CreER ${ }^{T 2}$ on the $\mathrm{Y}$ chromosome. Here, using third generation sequencing, we physically mapped the Myh11-CreER ${ }^{T 2}$ transgene and surmise that it integrated initially at the terminal end of the $X$ chromosome near the PAR, where high rates of recombination occur. ${ }^{47}$ The Myh11-CreER ${ }^{T 2}$ transgene then translocated to the $\mathrm{Y}$ chromosome carrying at least two $\mathrm{X}$ linked genes, which we observe to be more abundantly expressed in Myh11-CreER ${ }^{T 2}$ mice. The insertion site on this "neo Y chromosome" has not been pinpointed as yet, but it must be close to the pseudoautosomal boundary on chromosome $Y$ to explain the $\sim 3 \%$ of cases of $\mathrm{t}(\mathrm{Y} ; \mathrm{X})$ we and others have seen. ${ }^{46}$ There likely exists several forms of the Myh11-CreER $R^{T 2}$ driver due to stochastic recombination and probable variation in breakpoints that could lead to spurious reporting of a conditional knockout in SMCs. We suggest future generation of Cre (or other recombinase) mice use a more targeted approach for single copy integration, either within a gene of interest or safe harbor locus on an autosome.

Despite the advantages of the Itga8-CreER ${ }^{T 2}$ mouse allowing for sex studies in a more VSMC-restrictive manner and without the overestimation of recombination stemming from a leaky CreER $R^{T 2}$, there are some limitations that need mention. First, as loss of both functional 
Itga8 alleles results in postnatal lethality, ${ }^{36}$ it will not be useful to breed the Itga8-CreER ${ }^{T 2}$ line to homozygosity. Unless the site of integration is known, breeding to homozygosity is ill-advised since there is the possibility of disrupting a gene (coding or noncoding) or critical regulatory sequence. ${ }^{48}$ Second, as with all other SMC Cre drivers currently in use, Itga8-CreER $R^{T 2}$ exhibits activity in non-VSMC types, most notably glomerular cells of the kidney. Although attempts to define these cell types were inconclusive, previous work suggests they are likely to be mesangial cells. ${ }^{28}$ Thus, when using the Itga8-CreER ${ }^{T 2}$ mouse for conditional VSMC knockouts, some assessment of renal function should be done. In this report, there was no evidence of proteinuria and histology of the kidney in Srf mutant mice revealed normal glomerular and tubular structures. Whether a glomerular phenotype is manifest under stress conditions awaits further study. Third, since Itga8 appears to be down-regulated under conditions of SMC dedifferentiation, ${ }^{30}$ the Itga8-CreER ${ }^{T 2}$ mouse may not be optimal for studies requiring inducible activity following vascular insult. On the other hand, there is evidence for the up-regulation of ITGA8 protein late in intimal disease. ${ }^{49}$ Further work in various vascular disease models will resolve this question. Fourth, the full activity profile of Itga8-CreER ${ }^{T 2}$ during embryonic development and in other non-VSMC types or VSMC-related pericytes, where activity of Myh11CreER $R^{T 2}$ has recently been demonstrated, ${ }^{50}$ is not known at this time. Continued characterization of Itga8-CreER ${ }^{T 2}$ under baseline and disease conditions will provide further insight into its utility in elucidating gene function in VSMCs. Finally, essentially nothing is known about the transcriptional/post-transcriptional control of Itga8. Transgenic mouse and in vitro luciferase assays failed to show the importance of a conserved SRF-binding site located 4kilobases upstream of the start site of Itga8 transcription. ${ }^{33}$ Remarkably, ectopic expression of the SRF coactivator, MYOCD, induced Itga8 expression in an SRF-independent manner. ${ }^{33}$ Genome-wide MYOCD binding studies could illuminate novel enhancers that confer higher expression of Itga8 in vascular versus visceral SMCs. Elucidating enhancers of Itga8 might inform the molecular basis for preferential expression of this integrin gene in VSMCs and the further development and refinement of Cre driver mice for VSMC conditional knockout models.

In summary, the Itga8-CreER ${ }^{T 2}$ mouse allows for sex-based studies with preferential activity in VSMCs, an attribute that has yet to be demonstrated in any other SMC-restricted Cre driver. As such, the Itga8-CreER $R^{T 2}$ mouse represents a solution to the problem of competing phenotypes arising from the inactivation of critical genes in visceral SMCs. 


\section{Methods}

\section{Mouse models}

The Myh11-CreER ${ }^{T 2}$ mouse (stock 019079) ${ }^{39}$ and $m T / m G$ reporter (stock 007676) ) $^{51}$ were obtained from the Jackson laboratory. The Sm22-Cre and floxed Srf strains have been described previously. ${ }^{38}$ To generate the Itga8-CreER ${ }^{T 2}$ allele, a DNA fragment containing CreER ${ }^{T 2}-P 2 A-m C h e r r y$ coding sequence and SV40 polyadenylation signal (CreER ${ }^{T 2}-P 2 A-$ $m$ Cherry-pA) was synthesized by GENEWIZ. A 0.7-kb 5'-homology arm containing 5'-upstream and 5'-untranslated region sequences of exon 1 of Itga8 and a 1.1-kb 3'-homology arm containing the coding sequence of exons 1-2 and intron 1-2 of Itga8 were PCR-amplified from C57BL/6J genomic DNA using 5'-primer set (5'-GTACTGGTGAGGAAGAGATCCTGTTGTCT-3' and 5'-CCCTCCTTCCCGAACGCTGTTCA-3') and 3' primer set (5'-

CGATGTCTGCGGGAACCCACTGTC-3' and 5'-

GCTGTGGGTTTCTACTGGTCCCAAGCACCT-3'), respectively. The homology arms were cloned, along with an FRT flanked pGK-Neo, pGK-DTA, and CreER ${ }^{T 2}-P 2 A-m C h e r r y-p A$, into pBluescript SKII (+) to generate the Itga8-CreER ${ }^{T 2}$ knock-in construct (Figure I in the Data Supplement). The targeting construct was linearized with $\mathrm{Ascl}$ and electroporated into C57BL/6J-129S6 hybrid G4 embryonic stem cells (ESCs) (a gift from Dr. Andras Nagy). ${ }^{52}$ The targeted Itga8-CreER ${ }^{T 2}$ ESC clones were confirmed by long and accurate PCR genotyping using external primers to identify the $1.0 \mathrm{~kb} 5^{\prime}$ and $1.3 \mathrm{~kb} 3^{\prime}$ targeted DNA fragments, and were microinjected into C57BL/6J blastocysts to generate mouse chimeras. By crossing the chimeras with flippase-expressing FLPeR mice in C57BL/6J background (Jackson Laboratory, 009086), the Neo cassette was removed to produce Itga8-CreER $R^{T 2}$ mouse line. The PCR primers used to identify the 5'- and 3'-homologous recombination events in the positively targeted ESCs and mice were: forward 5'-

GCCTACACTTAAATTCTGTGTGACCTCAGAGTCATGT-3') reverse (5'CCATCAGGTTCTTGCGAACCTCATCACTCGT-3'), and forward (5'-CATGCTCCAGACTGC CTTG-3') and reverse (5'-GGCAGGAGCTGAGGCCATAGCCTTCAC-3'), respectively. The final integrated construct was verified by Sanger sequencing both strands. Mice are currently in the N14 generation following successive back-crossing to wild type C57BL/6J mice. The inducible Myocd mouse (iMyocd) was generated in the same ESC as above with an HA-tagged 
Myocd_v1 cDNA ${ }^{53}$ cloned downstream of a CMV-driven stop-floxed cassette in the Rosa26 locus.

\section{Tamoxifen administration}

Cre mouse lines were crossed with $m T / m G$ fluorescent reporter mice, a floxed Srf mouse, ${ }^{38}$ or an inducible Myocd mouse, as described. Inducible Cre activity was achieved by intraperitoneal injection of tamoxifen (Sigma-Aldrich, T5648) mixed and sonicated in $100 \%$ ethanol/sunflower seed oil (Sigma-Aldrich, S5007) at 1:9, v/v. The same dose of tamoxifen (40 $\mathrm{mg} / \mathrm{kg}$, ip) was delivered daily over a 5 -day period, followed by a washout of tamoxifen for at least 10 days before experimental measures.

\section{Confocal microscopy}

For frozen samples, fresh tissues were rapidly isolated and fixed overnight in $4 \%$ paraformaldehyde. Tissues were then cryoprotected over a second night with $30 \%$ sucrose followed by embedding in Optimal Cutting Temperature (OCT) compound (VWR, 102094-106). $5 \mu \mathrm{m}-7 \mu \mathrm{m}$ sections were cut with a cryostat (Leica Biosystems, CM 1950) and kept frozen until imaging was performed. Slides were removed from $-20^{\circ} \mathrm{C}$ and warmed to room temperature. OCT compound was removed by washing with 1xPBS for 3 times. Auto-fluorescence was quenched with Vector ${ }^{\circledR}$ TrueVIEW $^{\circledR}$ Autofluorescence Quenching Kit (Vector Laboratories, SP8500-15) and the slides cover-slipped with ProLong ${ }^{\text {TM }}$ Gold Antifade Mountant (Thermo, P36930) containing DAPI stain and air dried. Imaging was done on either an Olympus IX81 or Zeiss LSM900 confocal microscope with 20x, 40x oil (z-stacked), and 60x oil (z-stacked) objectives. Laser settings for confocal imaging were kept constant throughout. Final images were cropped to their published size in Adobe ${ }^{\circledR}$ Photoshop ${ }^{\circledR}$ with no digital enhancements, save the Testis panel corresponding to Itga8-CreER ${ }^{T 2}$ in supplementary Figure $6 \mathrm{~b}$; the latter panel was enhanced uniformly to increase brightness. For paraffin sections, tissues were isolated and immersion-fixed in methanol- $\mathrm{H}_{2} \mathrm{O}$-acetic acid $(60 / 30 / 10, \mathrm{v} / \mathrm{v})$ overnight, processed in a benchtop tissue processor (Leica Biosystems, TP1020), and embedded (Sakura, Tissue Tek TEC 5) in paraffin blocks. Sections were cut with a microtome (Microm, HM $355 \mathrm{~S}$ ) at $5 \mu \mathrm{m}$ and baked overnight at $58^{\circ} \mathrm{C}$. Slides were then deparaffinized in a Leica ${ }^{\circledR}$ Autostainer XL (Leica Biosystems, ST5010), stained with antibodies (Supplementary Table 3), quenched with Vector ${ }^{\circledR}$ 
TrueVIEW ${ }^{\circledR}$ Autofluorescence Quenching Kit (Vector Laboratories, SP-8500-15), and coverslipped with ProLong ${ }^{\text {TM }}$ Gold Antifade Mountant. Imaging was done on an Olympus IX81 or Zeiss LSM900 confocal microscope with 20x, 40x oil (z-stacked), and 60x oil (z-stacked) objectives. Final paraffin sectioned images were processed in Photoshop ${ }^{\circledR}$ without any enhancements.

\section{Recombination efficiency}

Several floxed mouse models carrying either Itga8-CreER ${ }^{T 2}$ or Myh11-CreER $R^{T 2}$ were treated with oil or tamoxifen as above. Following a standard 10-14 day washout period, tissues (aorta and bladder) were isolated for quantitative PCR of genomic DNA ( $m T / m G$ reporter) and Western blotting for the inducible Myocardin and floxed Srf mice, respectively. For popliteal vascular recombination efficiencies, mice were anesthetized with an injection of ketamine/xylazine (25/2.5 mg/ml, i.p.) and their legs shaved and cleaned. The saphenous artery and vein and the popliteal lymphatic vessel were excised from the tissue and kept in Krebs buffer with $0.5 \% \mathrm{BSA}$ at $4^{\circ} \mathrm{C}$ until imaged. Each vessel was then cannulated and pressurized in an imaging chamber filled with Krebs buffer for further removal of adipose and connective tissue. Vessels were then equilibrated in calcium free solution for 15 minutes to prevent movement during live imaging. At least 2 separate fields of view of each vessel were acquired with a Yokagawa CSU-X Spinning Disc Confocal Microscope on an inverted Olympus IX81 with a Hamamatsu Flash4 camera using both 20x and 40x objectives. Each live vessel acquisition was a Z-stack from the bottom edge of the vessel to the midpoint for both the GFP (488nm) and tdTomato wavelengths $(561 \mathrm{~nm})$.

Following the live image acquisition, the lymphatic popliteal vessels were fixed at room temp with ice cold 4\% PFA for 15 minutes. The vessels were then washed with PBS and stored at $4 \mathrm{C}$ for subsequent immunostaining. The fixed vessels were permeabilized with PBST $(0.3 \%$ triton) for 1 hour and then blocked for 3 hours with BlockAid (Thermofisher). Vessels were stained overnight with anti-GFP at 1:200 (rabbit, ThermoFisher A-11122) and anti-smooth muscle actin at 1:500 (mouse, Sigma A5228) in Blockaid buffer. The vessels were then washed with PBS and stained with Donkey anti-rabbit AF488 (A-21206, Thermofisher) and Donkey antimouse AF647 at 1:200 (A-31571, Thermofisher) for 1 hour at 4C. Vessels were washed in PBS and then recannulated and imaged as described above. 
To assess recombination efficiency in the smooth muscle layer of the saphenous vein and artery, the number of circumferential vascular muscle cells expressing either $\mathrm{GFP}^{+}$or tdTomato ${ }^{+}$were tabulated from Z-stacks from $40 \mathrm{x}$ acquisitions. To assess recombination efficiency in the smooth muscle layer of popliteal lymphatic vessels the number of GFP+SMA ${ }^{+}$ and GFP-SMA ${ }^{+}$lymphatic muscle cells were counted and tabulated from Z-stacks acquired at 40X. To create the differential images used as a visual aid, the RFP Z-stack and GFP Z-stack were converted to 32 bit images, the RFP Z-stack was divided by the GFP Z-stack in FIJI and a maximum projection was made of the resulting stack.

\section{Western blotting}

Harvested cells or pulverized frozen tissues were washed with cold 1XPBS and lysed with Cell Lysis Buffer (Cell Signaling, 9803) containing complete protease inhibitor cocktail (SigmaAldrich, 4693132001). Protein concentration was determined (Bio-Rad, 5000202) and samples were boiled with NuPAGE LDS buffer (Thermo, NP0008). Samples were run in 4-20\% SDSPAGE gel (Bio-Rad, 4561094) in 1x Tris-Glycine running buffer (Bio-Rad, A0028). Proteins were transferred to PVDF membrane using Trans-blot Turbo transfer system and compatible transfer kit (Bio-Rad, 1704272). Membranes were blocked in blocking buffer (Bio-Rad, 12010020) and incubated with appropriate antibodies (Supplementary Table 3). Finally, chemiluminescent substrate (Bio-Rad, 1705061) and ChemiDoc imaging system (Bio-Rad, 12003154) were used for digital imaging of Western blots. Urine from Itga8-CreER ${ }^{T 2} / \mathrm{Srf} f / f l$ and Itga8-CreER ${ }^{T 2} / \mathrm{Srf} \mathrm{fl}^{\mathrm{fl}+}$ mice was collected and denatured in NuPAGE LDS buffer. Samples were loaded onto SDS-PAGE gel and run in 1x Tris-Glycine running buffer. Gels were stained with Coomassie blue and images were documented as above. Purified bovine serum albumin was run alongside the urine samples and served as a positive control for the presence of protein.

\section{RNA isolation and real time quantitative RT-PCR}

Total RNA was extracted using QIAzol ${ }^{\circledR}$ Lysis Reagent (Qiagen, 79306) followed by isopropanol precipitation and $70 \%$ ethanol washing. GlycoBlue ${ }^{\mathrm{TM}}$ Coprecipitant was applied for single aortae to facilitate the handling of small quantities of RNA. Air-dried RNA pellets were dissolved in RNase-free water. Reverse transcription was performed using Bio-Rad iScript cDNA synthesis kit (Bio-Rad, 1708891) after DNase I treatment (Thermo, AM2222). Real time 
quantitative PCR was performed using iTaq Universal SYBR Green Supermix (Bio-Rad, 1725121) with primers listed in Supplementary Table 3. Following delta delta Ct normalization to a house-keeping control, levels of normalized gene expression were plotted as fold-change from control samples.

\section{Bulk RNA-seq}

Itga8-Cre/Srf ${ }^{f / f l}$ and Itga8-Cre/Srf fl/+ mice ( $\mathrm{n}=3$ mice each) were administered tamoxifen at six weeks of age as above. Four weeks after the first dose of tamoxifen, aortae were collected and homogenized in RNeasy Plus Micro Kit with genomic DNA eliminator columns (Qiagen, 74034). Total RNA library preparation (TruSeq stranded) and high-throughput RNAseq were performed on an Illumina HiSeq 4000 by Genomics Research Core of the University of Rochester. Gene Ontology analysis was done with DAVID ${ }^{54}$ and over-representation of DNA binding sites was determined with oPOSSUM. ${ }^{55}$ RNA-seq data have been submitted to the Gene Expression Omnibus (GSE138824). In a separate experiment, aortas from male wild type mice or age-matched mice with either Itga8-CreER $R^{T 2}$ or Myh11-CreER $R^{T 2}(\mathrm{n}=3)$ were processed for total RNA isolation, library preparation, and sequencing on the Illumina HiSeq 4000 at the University of Rochester. Data were analyzed as described previously ${ }^{56}$ and submitted to the Gene Expression Omnibus (TBA).

\section{Nuclear-cytoplasmic RNA fractionation for LncRNA expression}

Cultured mouse vascular smooth muscle cells (MOVAS) ${ }^{57}$ were fractionated using Protein and RNA isolation system kit according to manufacturer's protocol (Thermo Fisher, AM1921). Real-time qRT-PCR was done with primers to the LncRNA upstream of Itga8 as well as Actb primers, used as a cytoplasmic marker (Supplementary Table 3).

\section{Flow Cytometry}

Whole blood samples were collected from wild-type, $m T m G$, Myh11-CreER ${ }^{T 2} / m T m G$, Sm22-Cre/mTmG, and Itga8-CreER ${ }^{T 2} / m T m G$ mice and centrifuged for 5 min at 2000x g at room temperature. Buffy coats were carefully transferred to a fresh $1.5 \mathrm{ml}$ microfuge tube. $500 \mathrm{ul}$ of $1 \mathrm{x}$ ACK Lysing Buffer (Thermo, A1049201) was added to lyse red blood cells. Samples were then centrifuged for $5 \mathrm{~min}$ at 3000x g at room temperature. Supernatants were carefully aspirated 
and the pellets were resuspended in 1x PBS. Cell suspensions were analyzed using Accuri C6 flow cytometer (BD Biosciences) in Flow Cytometry Core of University of Rochester. Signals from FITC channel were collected and gated for GFP positive cells from each experimental condition.

\section{Radiotelemetry recording of blood pressure}

All physiological studies used male and female mice homozygous for floxed Srf and heterozygous for Itga8-CreER $R^{T 2}$ ( \pm tamoxifen). Following a 10 day washout period from last tamoxifen (or oil) injection, male and female mice (13 weeks) were implanted with indwelling telemeters (carotid catheter) (DSI ${ }^{\odot}$ Model \#PA-C10, New Brighton, MN) under isoflurane anesthesia as previously described. ${ }^{58}$ After a 7-day recovery period, baseline blood pressure (BP) (mean arterial pressure, systolic and diastolic pressure) and heart rate measurements were consciously recorded for 7 days. Mice were then implanted with subcutaneous miniosmotic pumps (Alzet, model 1002, 14 day pump 0.25 ul/hour) containing angiotensin II (Phoenix Pharmaceuticals) diluted in isotonic saline at a dose of $490 \mathrm{ng} / \mathrm{kg} / \mathrm{min}$ as previously described. ${ }^{59}$ Blood pressure was recorded subsequently for 14 days.

\section{Vascular Reactivity}

Thoracic aortas from mice utilized for telemetry blood pressure recording protocol (ANGII-infused) were excised and cleaned of excess adipose tissue. Aortas were cut in $2 \mathrm{~mm}$ rings and mounted on pins of a DMT ${ }^{\circledR}$ wire myograph (Ann Arbor, MI) as previously described. ${ }^{58}$ Concentration response curves to phenylephrine ( $1 \mathrm{nM}-30 \mu \mathrm{M}$ concentrations) as well as maximum responses to $\mathrm{KCl}(80 \mathrm{mM})$ were performed and recorded with LabChart $^{\circledR}$ analysis software (AD Instruments ${ }^{\circledR}$, Colorado Springs, $\left.C O\right)$.

\section{Mapping and copy number determination of Myh11-CreER ${ }^{\mathrm{T}}$}

Long-read libraries of (i) Ultra-Long DNA Sequencing (SQK-ULK001) and (ii) CAS9 Sequencing (SQK-CS9109) kits from ONT Nanopore were prepared following manufacturer's instructions (www.nanoporetech.com). For Ultra-Long DNA libraries, ultrahigh molecular weight genomic DNA was isolated with Circulomics kits (NB-900-601-01 and NB-900-701-01) following manufacturer's instructions (www.circulomics.com). For CAS9 libraries, high molecular weight genomic DNA was isolated with NEB kit (T3060) following manufacturer's instructions (www.neb.com). Five males, including a male from 
Jackson Laboratory (www.jax.org/), were used as input for mapping following. ${ }^{60}$ For CAS9 Sequencing (CRISPR-LRS), crRNAs were designed with $\mathrm{CHOPCHOP61}$ using default parameters

(https://chopchop.cbu.uib.no) (Supplementary Table 3). Long-read libraries were run on R9.4.1 flow cells on a minlON Mk1B or a GridION Mk1 with fast5 to fastq read conversion using guppy (v4.2.2) on MinKNOW (v20.10.3) MinKNOW Core (v4.1.2) and fast base-calling option for the base-call model and minimum Q-score of 7 option for read filtering. Fastq files were mapped to reference sequences (improved Cre or segments of chromosome 16) using the Long-Read Support (beta) plugin in Qiagen CLC Genomics Workbench (www.qiagen.com) which uses open-source tool minimap2. Mapped reads (informative reads) were manually queried against NCBI nr/nt, refseq genome databases

(https://blast.ncbi.nlm.nih.gov/Blast.cgi) and UCSC genome browser with BLAT tool (https://genome.ucsc.edu), where a mini-tiled map of Myh11-CreER ${ }^{T 2}$ integration locus was constructed. ${ }^{60}$ To determine copy number of Myh11-CreER ${ }^{T 2}$, qPCR was used as above. Genomic DNA was diluted to $50 \mathrm{ng}$ for input. Two primer sets targeting $E R^{T 2}$ of Myh11-CreER ${ }^{T 2}$ were normalized to internal control primers (Supplementary Table 3). Real time quantitative PCR conditions were the following: step $195^{\circ} \mathrm{C}$ for $3 \mathrm{~min}$; step $295^{\circ} \mathrm{C}$ for $30 \mathrm{sec}, 60^{\circ} \mathrm{C}$ for $30 \mathrm{sec}, 72^{\circ} \mathrm{C}$ for $30 \mathrm{sec}$ for 40 cycles; melt curve analysis.

\section{Data availability}

Long-read data (informative reads) have been submitted to NCBI SRA database (www.ncbi.nlm.nih.gov/sra) under BioProject number (TBA).

\section{Statistical analysis}

A Shapiro-Wilk test was performed to determine whether data were normally distributed. Paired $t$-test was used for comparisons between experimental and control conditions or oneand two-way ANOVA for multiple group comparisons with Tukey's post-hoc test for individual comparisons. All data analyses were performed in GraphPad Prism 8 (GraphPad Software). Results are expressed as mean \pm standard deviation unless specified otherwise. Probability values of $p<0.05$ were considered statistically significant. 


\section{Acknowledgments}

We thank members of the University of Rochester Medical Center Genomics Research Core for carrying out the RNA-seq experiments.

\section{Sources of Funding}

This work was supported by the American Heart Association post-doctoral grants 17POST3360938 to QL and CDA858380 to JLF; and National Institutes of Health grants R00HL146948 to JFL; HL155265 to EBC; HL122578 to MJD; HL122686 and HL139794 to XL; HL138987, HL136224, and HL147476 to JMM; EY026614 to LG; R00-HL143198 to SDZ;

\section{Disclosures}

None

\section{Author Contributions}

GDW developed the figures and performed Western blotting studies in Supplementary Figs. $4 \mathrm{e}$ and $9 \mathrm{a}$ and Figs. $2 \mathrm{~b}$ and $3 \mathrm{~g}$-3h, bulk RNA-seq studies in Fig. $2 \mathrm{e}$ and Supplementary Table 2 , and assisted with the physiological measurements in Figs. 4-6; JLF performed and analyzed vascular function and blood pressure data of Figs. 4, 5 and 6a-6c and wrote the methods section related to these experiments; JD performed the studies in Fig. $3 \mathrm{c}$ and $3 \mathrm{~d}$ and conducted tamoxifen injections for studies blood pressure and myography measurements; ARG provided foundational work in the beginning and helped generate Supplementary Fig. 1; PG assisted with Western blotting studies of Supplementary Fig. 4a, 4b; ACY generated the data for Supplementary Fig. 3; OJS performed all histology, immunostaining, confocal immunofluorescence and bright-field microscopy; MJD and SDZ planned and performed experiments in Fig. $3 e$ and $3 \mathrm{f}$ and Supplementary Fig. 8 showing recombination efficiency of Myh11-CreER $R^{T 2}$ and Itga8-CreER $R^{T 2}$ in blood and lymphatic vasculature beds, and they contributed to the writing and editing of the methods and results; CKC, ACY, and SHG did all breeding and genotyping of mice; CKC provided images to Supplementary figures 9c and 11a; CTB and TCK helped perform the telemetry experiments of Fig. 4 and 5; AA helped with the interpretation of Myh11-CreER ${ }^{T 2}$ mapping and the design of Fig. 2d; WBB prepared and analyzed the Sanger sequencing of the Cre in Myh11-CreER ${ }^{T 2}$ of Supplementary Fig. 7 and the 
long read sequence mapping of Myh11-CreER ${ }^{T 2}$ in Fig. 2c and 2d; AK contributed to Western blotting studies of Supplementary Fig. 4a, 4b and Fig. 2b; XL contributed to data in Fig. 2a and provided intellectual input into study design and interpretation; $L G$ and $X X$ designed and generated the Itga8-CreER ${ }^{T 2}$ mouse and developed Supplementary Fig. 2; EBC helped with the acquisition and interpretation of the blood pressure and vascular reactivity data; QL performed the studies in Supplementary Figs. 4, 5, 10b-10g, and 11b, 11d and Fig. 7; he also provided intellectual input throughout the study and finalized the figures; JMM conceived and supervised the entire project, analyzed all data, and outlined, wrote, and edited the manuscript. 


\section{References}

1. Sauer, B. Inducible gene targeting in mice using the Cre/lox system. Methods 14, 381392 (1998).

2. Meinke, G., Bohm, A., Hauber, J., Pisabarro, M.T. \& Buchholz, F. Cre recombinase and other tyrosine recombinases. Chem Rev 116, 12785-12820 (2016).

3. Gu, H., Marth, J.D., Orban, P.C., Mossmann, H. \& Rajewsky, K. Deletion of a DNA polymerase beta gene segment in T cells using cell type-specific gene targeting. Science 265, 103-106 (1994).

4. Nagy, A. Cre recombinase: the universal reagent for genome tailoring. Genesis 26, 99109 (2000).

5. Majesky, M.W. Developmental basis of vascular smooth muscle diversity. Arteriosclerosis, Thrombosis and Vascular Biology 27, 1248-1258 (2007).

6. Tasian, G., Cunha, G. \& Baskin, L. Smooth muscle differentiation and patterning in the urinary bladder. Differentiation 80, 106-117 (2010).

7. Somlyo, A.P. \& Somlyo, A.V. Signal transduction and regulation in smooth muscle. Nature 372, 231-236 (1994).

8. Sanders, K.M., Kito, Y., Hwang, S.J. \& Ward, S.M. Regulation of gastrointestinal smooth muscle function by interstitial cells. Physiology (Bethesda) 31, 316-326 (2016).

9. Kuriyama, H., Kitamura, K., Itoh, T. \& Inoue, R. Physiological features of visceral smooth muscle cells, with special reference to receptors and ion channels. Physiol Rev 78, 811920 (1998).

10. Owens, G.K., Kumar, M.S. \& Wamhoff, B.R. Molecular regulation of vascular smooth muscle cell differentiation in development and disease. Physiological Reviews 84, 767801 (2004).

11. Chakraborty, R. et al. Promoters to study vascular smooth muscle: mistaken identity? Arteriosclerosis, Thrombosis and Vascular Biology 39, 603-612 (2019).

12. Angstenberger, M. et al. Severe intestinal obstruction on induced smooth muscle-specific ablation of the transcription factor SRF in adult mice. Gastroenterology 133, 1948-1959 (2007).

13. Mericskay, M. et al. Inducible mouse model of chronic intestinal pseudo-obstruction by smooth muscle-specific inactivation of the SRF gene. Gastroenterology 133, 1960-1970 (2007).

14. He, W.Q. et al. Myosin light chain kinase is central to smooth muscle contraction and required for gastrointestinal motility in mice. Gastroenterology 135, 610-620 (2008).

15. Albinsson, S. et al. Smooth muscle miRNAs are critical for post-natal regulation of blood pressure and vascular function. PLoS One 6, e18869 (2011).

16. Park, C. et al. Serum response factor-dependent microRNAs regulate gastrointestinal smooth muscle cell phenotypes. Gastroenterology 141, 164-175 (2011).

17. Huang, J. et al. Myocardin is required for maintenance of vascular and visceral smooth muscle homeostasis during postnatal development. Proceedings of the National Academy of Sciences USA 112, 4447-4452 (2015).

18. Morano, I. et al. Smooth-muscle contraction without smooth-muscle myosin. Nature Cell Biology 2, 371-375 (2000).

19. Li, S., Wang, D.Z., Richardson, J.A. \& Olson, E.N. The serum response factor coactivator myocardin is required for vascular smooth muscle development. Proceedings of the National Academy of Sciences of the United States of America 100, 9366-9370 (2003). 
20. Niessen, P. et al. Smoothelin-a is essential for functional intestinal smooth muscle contractility in mice. Gastroenterology 129, 1592-1601 (2005).

21. Halim, D. et al. Loss of LMOD1 impairs smooth muscle cytocontractility and causes megacystis microcolon intestinal hypoperistalsis syndrome in humans and mice. Proceedings of the National Academy of Sciences USA 114, E2739-E2747 (2017).

22. Guzzardo, P.M. et al. A small cassette enables conditional gene inactivation by CRISPR/Cas9. Sci Rep 7, 16770 (2017).

23. Gurumurthy, C.B. et al. Reproducibility of CRISPR-Cas 9 methods for generation of conditional mouse alleles: a multi-center evaluation. Genome Biol 20, 171 (2019).

24. Horii, T. et al. Efficient generation of conditional knockout mice via sequential introduction of lox sites. Sci Rep 7, 7891 (2017).

25. Anzalone, A.V. et al. Search-and-replace genome editing without double-strand breaks or donor DNA. Nature 576, 149-157 (2019).

26. Bossy, B., Bossy-Wetzel, E. \& Reichardt, L.F. Characterization of the integrin a8 subunit: a new integrin b1-associated subunit, which is prominently expressed on axons and on cells in contact with basal laminae in chick embryos. EMBO Journal 10, 2375-2385 (1991).

27. Schnapp, L.M., Breuss, J.M., Ramos, D.M., Sheppard, D. \& Pytela, R. Sequence and tissue distribution of the human integrin a8 subunit: a b1-associated a subunit expressed in smooth muscle cells. Journal of Cell Science 108, 537-544 (1995).

28. Hartner, A., Schocklmann, H., Prols, F., Muller, U. \& Sterzel, R.B. Alpha8 integrin in glomerular mesangial cells and in experimental glomerulonephritis. Kidney International 56, 1468-1480 (1999).

29. Chen, J., Maltby, K.M. \& Miano, J.M. A novel retinoid-response gene set in vascular smooth muscle cells. Biochem Biophys Res Commun 281, 475-482 (2001).

30. Zargham, R. \& Thibault, G. a8b1 integrin expression in the rat carotid artery: involvement in smooth muscle cell migration and neointima formation. Cardiovascular Research 65, 813-822 (2005).

31. Zargham, R., Touyz, R.M. \& Thibault, G. a8 integrin overexpression in de-differentiated vascular smooth muscle cells attenuates migratory activity and restores the characteristics of the differentiated phenotype. Atherosclerosis 195, 303-312 (2007).

32. Wang, D.Z. et al. Activation of cardiac gene expression by myocardin, a transcriptional cofactor for serum response factor. Cell 105, 851-862 (2001).

33. Kitchen, C.M., Cowan, S.L., Long, X. \& Miano, J.M. Expression and promoter analysis of a highly restricted integrin alpha gene in vascular smooth muscle. Gene 513, 82-89 (2013).

34. Miano, J.M. Serum response factor: toggling between disparate programs of gene expression. Journal of Molecular Cellular Cardiology 35, 577-593 (2003).

35. Feil, R., Wagner, J., Metzger, D. \& Chambon, P. Regulation of Cre recombinase activity by mutated estrogen receptor ligand-binding domains. Biochem Biophys Res Commun 237, 752-757 (1997).

36. Muller, U. et al. Integrin a8b1 is critically important for epithelial-mesenchymal interactions during kidney morphogenesis. Cell 88, 603-613 (1997).

37. Shen, Z. et al. Smooth muscle protein 22 alpha-Cre is expressed in myeloid cells in mice. Biochemical Biophysical Research Communications 422, 639-642 (2012). 
38. Miano, J.M. et al. Restricted inactivation of serum response factor to the cardiovascular system. Proceedings of the National Academy of Sciences USA 101, 17132-17137 (2004).

39. Wirth, A. et al. G12-G13-LARG-mediated signaling in vascular smooth muscle is required for salt-induced hypertension. Nature Medicine 14, 64-68 (2008).

40. Alvarez-Aznar, A. et al. Tamoxifen-independent recombination of reporter genes limits lineage tracing and mosaic analysis using CreER(T2) lines. Transgenic Res 29, 53-68 (2020).

41. Shimshek, D.R. et al. Codon-improved Cre recombinase (iCre) expression in the mouse. Genesis 32, 19-26 (2002).

42. Retailleau, K. et al. Selective involvement of serum response factor in pressure-induced myogenic tone in resistance arteries. Arteriosclerosis, Thrombosis and Vascular Biology 33, 339-346 (2013).

43. Galmiche, G. et al. Inactivation of serum response factor contributes to decrease vascular muscular tone and arterial stiffness in mice. Circ Res 112, 1035-1045 (2013).

44. Sandlesh, P., Juang, T., Safina, A., Higgins, M.J. \& Gurova, K.V. Uncovering the fine print of the CreERT2-LoxP system while generating a conditional knockout mouse model of Ssrp1 gene. PLoS One 13, e0199785 (2018).

45. Clayton, J.A. Applying the new SABV (sex as a biological variable) policy to research and clinical care. Physiol Behav 187, 2-5 (2018).

46. Liao, M. et al. An X-linked Myh11-CreER(T2) mouse line resulting from $Y$ to $X$ chromosome-translocation of the Cre allele. Genesis 55 (2017).

47. Morgan, A.P., Bell, T.A., Crowley, J.J. \& Pardo-Manuel de Villena, F. Instability of the pseudoautosomal boundary in house mice. Genetics 212, 469-487 (2019).

48. Goodwin, L.O. et al. Large-scale discovery of mouse transgenic integration sites reveals frequent structural variation and insertional mutagenesis. Genome Res 29, 494-505 (2019).

49. Zargham, R., Pepin, J. \& Thibault, G. a8b1 integrin is up-regulated in the neointima concomitant with late luminal loss after balloon injury. Cardiovascular Pathology $16212-$ 220 (2007).

50. Hess, D.L. et al. Perivascular cell-specific knockout of the stem cell pluripotency gene Oct4 inhibits angiogenesis. Nat Commun 10, 967 (2019).

51. Muzumdar, M.D., Tasic, B., Miyamichi, K., Li, L. \& Luo, L. A global double-fluorescent Cre reporter mouse. Genesis 45, 593-605 (2007).

52. George, S.H. et al. Developmental and adult phenotyping directly from mutant embryonic stem cells. Proceedings of the National Academy of Sciences USA 104, 4455-4460 (2007).

53. Imamura, M., Long, X., Nanda, V. \& Miano, J.M. Expression and functional activity of four myocardin isoforms. Gene 464, 1-10 (2010).

54. Dennis, G., Jr. et al. DAVID: database for annotation, visualization, and integrated discovery. Genome Biology 4, 3 (2003).

55. Ho Sui, S.J., Fulton, D.L., Arenillas, D.J., Kwon, A.T. \& Wasserman, W.W. oPOSSUM: integrated tools for analysis of regulatory motif over-representation. Nucleic Acids Research 35, W245-W252 (2007).

56. Gao, P. et al. Prime editing in mice reveals the essentiality of a single base in driving tissue-specific gene expression. Genome Biol 22, 83 (2021). 
57. Afroze, T. et al. Calcineurin-independent regulation of plasma membrane Ca2+ ATPase4 in the vascular smooth muscle cell cycle. American Journal of Physiology Cell Physiology 285, C88-C95 (2003).

58. Faulkner, J.L. et al. Lack of suppression of aldosterone production leads to salt-sensitive hypertension in female but not male Balb/C mice. Hypertension 72, 1397-1406 (2018).

59. Wu, J. et al. Inflammation and mechanical stretch promote aortic stiffening in hypertension through activation of p38 mitogen-activated protein kinase. Circ Res 114, 616-625 (2014).

60. Bryant, W.B. et al. CRISPR-LRS for mapping transgenes in the mouse genome. bioRxiv, 2022.2001.2005.475144 (2022).

61. Montague, T.G., Cruz, J.M., Gagnon, J.A., Church, G.M. \& Valen, E. CHOPCHOP: a CRISPR/Cas9 and TALEN web tool for genome editing. Nucleic Acids Res 42, W401-407 (2014). 
Table. Summary of differences between two SMC Cre drivers

\begin{tabular}{|l|l|}
\hline \multicolumn{1}{|c|}{ Itga8-CreER } & \multicolumn{1}{c|}{ Myh11-CreER } \\
\hline Single-copy knockin on Chr 2 & Multi-copy transgene on Chr $\mathrm{Y}$ \\
\hline Chromosome stability & Exhibits t(Y;X) at 3\% of pups \\
\hline Male-female studies & Male only studies \\
\hline Original Cre & Codon-optimized Cre \\
\hline Low level Cre expression & High level Cre expression \\
\hline More stable transcriptome & Altered transcriptome \\
\hline Undetectable Cre leakiness & Cre leakiness \\
\hline Active primarily in VSMCs & Active in all SMC lineages \\
\hline Circumvents visceral myopathies & Some lethal visceral myopathies \\
\hline Active in mesangial cells of kidney & Active in cells of thymus \\
\hline Unknown pericyte activity & Active in some pericytes \\
\hline
\end{tabular}




\section{Figure Legends}

\section{Fig. 1 Comparative activity of Itga8-CreER ${ }^{T 2}$ versus Myh11-CreER ${ }^{T 2}$ in adult tissues.}

Paired tissues from 8-week old mice carrying indicated CreER $R^{T 2}$ and $m T / m G$ reporter. Images shown here and below are representative of tissues from both genders (Itga8-CreER ${ }^{T 2}$ only) obtained over a span of three years and multiple generations. Green fluorescent protein (GFP) staining reflects Cre-mediated excision of the membrane Tomato $(m T)$ reporter and is primarily confined to SMCs of each tissue. DAPI was applied where shown for nuclear staining. White arrows indicate blood vessels and white arrowheads indicate visceral smooth muscle cells. The scale bar represents $50 \mu \mathrm{m}$ for aorta and uterus and $100 \mu \mathrm{m}$ for all other panels.

Fig. 2 Distinguishing features of Myh11-CreER ${ }^{T 2}$. a, Tamoxifen-independent recombination activity in Myh11-CreER ${ }^{T 2}$ adult tissues. Arrowheads point to SMCs with leaky activity. b, Western blot of CreER ${ }^{T 2}$ protein with quantitation below. c, qPCR of copy number of Myh11CreER ${ }^{T 2}$ transgene from adult spleen. Two primer pairs (PCR 1 and PCR 2) were used that amplify non-overlapping sequences in $E R^{T 2}$. d, Schematic based on long read sequence mapping of Myh11-CreER ${ }^{T 2}$ transgene. The dashed lines indicate translocation from the $\mathrm{X}$ chromosome to $\mathrm{Y}$ chromosome carrying $\mathrm{X}$-linked genes surrounding the approximate insertion site of the transgene. Bent arrows indicate the transcription start site of each gene. Scale bar is an approximation. e, Bulk RNA-seq summary of differentially expressed genes in the absence of tamoxifen between wild type, Myh11-CreER ${ }^{T 2}$ and Itga8-CreER ${ }^{T 2}$ mice (n = 3 mice per genotype). Group 1 represents significantly elevated genes in Myh11-CreER ${ }^{T 2}$ aorta as compared to Itga8-CreER ${ }^{T 2}$ and wild type; Group 2 represents significantly elevated genes in wild type aorta; Group 3 represents significantly elevated genes in aorta Itga8-CreER ${ }^{T 2}$ aorta. A complete list of differentially expressed genes is provided in Supplementary Table 2.

Fig. 3 Recombination efficiency of Itga8-CreER ${ }^{T 2}$ versus Myh11-CreER ${ }^{T 2}$. a, Schematic of $m T / m G$ reporter at the Rosa26 locus and position of primers (arrows) for measuring recombination. b, qPCR of genomic DNA following Oil or Tamoxifen (Tam) administration. c, Similar study as above only an inducible Myocd transgenic mouse was bred to each CreER $R^{T 2}$ driver for oil (Ctrl) or Tam administration and Western blotting for the presence of the HA-tagged 
MYOCD protein. d, Quantitative data of Western blots in panel c; percent relative density to the TUBA1A loading control; $n=3$ independent mice per condition. e, Snapshot of live images of cultured popliteal lymphatic vessels from each CreER $R^{T 2}$ and $\mathbf{f}$, quantitation of GFP+ fluorescence in lymphatic muscle cells (LMCs); $n=4$ independent mice per genotype. g, Western blot of SRF protein in aorta and bladders of each indicated genotype with or without Tam administration. $\mathbf{h}$, Quantitative data of panel $\mathbf{g}$; percent relative density to the TUBA1A loading control; $n=3$ independent mice per condition.

Fig. 4 Chronic blood pressure measurements in Itga8-CreER ${ }^{T 2}$ mediated Srf knockout male mice. Effects of Srf conditional knockout (Srf cKO) on systolic blood pressure (a, b), diastolic blood pressure $(\mathbf{c}, \mathbf{d})$, mean arterial pressure $(\mathbf{e}, \mathbf{f})$ and heart rate $(\mathbf{g}, \mathbf{h})$ in male mice as measured by telemetry over 3 weeks. Control mice here and in Figures 5 and 6 are oil-treated, homozygous floxed Srf mice carrying Itga8-CreER ${ }^{T 2}$. $\mathrm{n}=3$ or 4 mice per condition.

Fig. 5 Chronic blood pressure measurements in Itga8-CreER ${ }^{T 2}$ mediated Srf knockout female mice. Effects of Srf conditional knockout (Srf cKO) on systolic blood pressure (a, b), diastolic blood pressure $(\mathbf{c}, \mathbf{d})$, mean arterial pressure $(\mathbf{e}, \mathbf{f})$ and heart rate $(\mathbf{g}, \mathbf{h})$ in female mice as measured by telemetry over 3 weeks. $n=3$ mice per condition.

Fig. 6 Vascular contractile competence in Itga8-CreER ${ }^{T 2}$ mediated Srf knockout mice. a, $\mathrm{KCl}$-stimulated contractile activity in female $(n=5)$ and male $(n=6)$ aorta under control or cKO condition. Male (b) and female (c) aortas treated with varying doses of Phenylephrine [Phe]. d, Confocal immunofluorescence microscopy of indicated proteins in control and Srf cKO male and female aorta. The scale bars are $50 \mu \mathrm{m}$.

Fig. 7 Bulk RNA-seq of aortas from Itga8-CreER ${ }^{T 2}$ mediated Srf knockout mice. a, Downregulated SMC contractile genes in aortas with conditional Srf knockout. b, Volcano plot of differentially expressed genes with conditional Srf knockout. c, oPOSSUM 3.0 analysis of overrepresented transcription factor binding sites in promoter/intron of top 250 down-regulated genes with conditional knockout of Srf. Enrichment scores for gene ontology terms (Molecular Function) related to downregulated (d) or upregulated (e) genes with conditional knockout of Srf. 


\section{Supplemental Figure Legends}

Supplementary Fig. 1, Tissue expression of indicated human genes. Screenshot of GTEx portal data of human SMC restricted genes obtained from https://www.gtexportal.org/home/gene/ITGA8. The boxed regions denote expression of each gene in the three vascular tissue types (tibial, aorta, and coronary).

Supplementary Fig. 2, Strategy and sequence validation of Itga8-CreER ${ }^{T 2}$. Top shows partial Itga8 locus and overlapping LncRNA as well as the strategy and component parts for mouse ESC targeting. The 22 amino acid P2A "self-cleaving" cassette (GSGATNFSLLKQAGDVEENPGP) should allow for concurrent detection of the CreER ${ }^{T 2}$ fusion and the mCherry reporter; however, mCherry fluorescence was not pursued in this study. Small horizontal arrows denote primers for PCR genotyping of mice. Bottom shows nucleotide sequence of targeted knockin cassette following Flippase-mediated removal of the Neo gene; colors correspond to the schematic at top. Note Kozak consensus sequence used for optimal Cre translation. The six blackened bases in Cre represent codon-optimized substitutions with one non-synonymous substitution, Ala2Ser. HR, homologous recombination.

Supplementary Fig. 3, Kidney agenesis in homozygous Itga8-CreER $R^{T 2}$ mice. Arrows denote adrenal glands. Note complete loss of kidneys in the homozygous mouse.

\section{Supplementary Fig. 4, ITGA8 and LncRNA expression in heterozygous Itga8-CreER ${ }^{T 2}$} mice. a, Western blotting of ITGA8 protein in two independent experiments each in triplicate with quantitative data at right. qRT-PCR analysis of Itga8 mRNA, b and the antisense Ak086420 LncRNA, c in wild type (WT) versus Itga8-CreER ${ }^{T 2}$ heterozygous aorta. d, qRT-PCR of cytosolic versus nuclear gene expression in wild type aorta. $n=4$ or more replicates per condition. $e$, Western blotting of ITGA8 and CreER ${ }^{\mathrm{T} 2}$ in aorta of age-matched male and female Itga8CreER $R^{T 2}$ heterozygous mice.

Supplementary Fig. 5, Activity of different SMC Cre mice in circulating cells. Flow cytometry of GFP labeled cells from a, wild type C57BL/6J mice; b, mTmG reporter mice; c, Sm22-Cre mice; d, Myh11-CreER ${ }^{T 2}$ mice; e, male Itga8-CreER ${ }^{T 2}$ mice; f, female Itga8-CreER ${ }^{T 2}$ 
mice. g-h, Quantitative data for GFP+ cells in upper left quadrant Q1 (g) and upper right quadrant $\mathrm{Q} 2(\mathbf{h})$ is shown for each Cre driver line ( $\mathrm{n}=3$ mice/line).

Supplementary Fig. 6, Comparative Cre activity in additional tissues. a, The GFP signal demonstrates recombination of the $m T / m G$ reporter in medial SMCs of both the Aorta and Vena Cava, whereas the red stained endothelium indicates the absence of recombination. Note the absence of autofluorescence in elastic lamellae of Aorta (see methods for how this was achieved). Scale bar is $33 \mu \mathrm{m}$ for Aorta and $100 \mu \mathrm{m}$ for Vena cava. The aorta and vena cava of Itga8-CreER ${ }^{T 2}$ was from a female mouse. b, GFP signal largely restricted to vascular SMC in blood vessels (arrows) of each indicated tissue type. Arrowheads represent GFP+ cells of the glomerulus in the kidney of an Itga8-CreER ${ }^{T 2}$ mouse. Note diffuse GFP+ signal in thymus of Myh11-CreER ${ }^{T 2}$ mouse, but not Itga8-CreER ${ }^{T 2}$ mouse. All images were processed exactly the same except for the Testis panel under Itga8-CreER ${ }^{T 2}$; here the image was enhanced in Photoshop to bring out more of the overall signal that otherwise would be too dark to visualize. Scale bar is $100 \mu \mathrm{m}$ for all images, save kidney where scale is $50 \mu \mathrm{m}$.

\section{Supplementary Fig. 7, Different Cre sequences between Myh11-CreER ${ }^{T 2}$ and Itga8-} CreER ${ }^{T 2}$ mice. Clustalw alignment of Sanger sequenced Cre for each driver mouse. Note Myh11-iCreER ${ }^{T 2}$ here (only) to indicate the improved codon usage for this Cre driver mouse. BLAST indicated 22\% nucleotide substitutions in Myh11-iCreER ${ }^{T 2}$ (yellow shaded) yielding $48 \%$ of codons optimized (red letters).

\section{Supplementary Fig. 8, Quantitative activity of Myh11-CreERT2 versus Itga8-CreER ${ }^{T 2}$ in} popliteal blood vessels. Confocal imaging of artery $(\mathbf{a}, \mathbf{b})$ and vein $(\mathbf{c}, \mathbf{d})$ with quantitation of each in panels e and f, respectively. Scale bars represent $100 \mu \mathrm{m}$.

\section{Supplementary Fig. 9, Intestinal phenotype in Myh11-CreER ${ }^{T 2}$ versus Itga8-CreER ${ }^{T 2}$} mediated knockout of serum response factor (Srf). a, Western blot showing lack of effect of tamoxifen on SRF protein levels in wild type aorta. b, Quantitation of panel a. c, Anatomy of abdominal cavity in the indicated genotypes of mice where Srf was inactivated. The two Srffl/fl/Myh11-CreER ${ }^{T 2}$ images were from mice 14 days following tamoxifen administration, 
whereas the Srfl/fl/Itga8-CreER ${ }^{T 2}$ image was from a mouse 8 weeks after tamoxifen administration. d, Oil (panels i-iii) and Tamoxifen (panels iv-vi) treated Srffl/fl/Myh11-CreER ${ }^{T 2}$ mice with dissected gross intestine (i, iv), H\&E stained intestine (ii, v), and immunostaining for SRF (green) and ACTA2 (red) in intestine (iii, vi). Scale bar is $100 \mu \mathrm{m}$ for ii, iii, v, and vi or $1 \mathrm{~mm}$ for i and iv.

\section{Supplementary Fig. 10, Itga8-CreER ${ }^{T 2}$ mediated inactivation of Srf in adult mouse tissues.}

a, Immunofluorescence confocal microscopy of sections of carotid artery (i, iv), bladder (ii, v), and intestine (iii, vi) from tamoxifen-treated male mice carrying homofloxed Srf alleles in the absence (i-iii) or presence (iv-vi) of Itga8-CreER ${ }^{T 2}$ (abbreviated Cre). Similar results were seen in the aorta and in vessels where one floxed Srf allele was excised with Itga8-CreER ${ }^{T 2}$ (data not shown). Sections were stained with antibodies to ACTA2 (red), SRF (green), and DAPI. Arrows and arrowheads point to blood vessels and visceral SMC, respectively. Scale bars is $20 \mu \mathrm{m}$. SRF positive nuclei were counted in sections of tamoxifen-administered homozygous floxed Srf mice without Cre (HoF+Tam, $n=6$ mice) or homozygous floxed Srf mice with Cre (HoF+Cre+Tam, $n=5$ mice) carotid arteries (b) and intestine ( $n=3$ mice each) (c). Western blots of SRF in aorta (d) and bladder (e) of indicated genotypes, all treated with same schedule of tamoxifen. Corresponding quantitative data is shown for mouse aorta $(\mathbf{f})$ and bladder $(\mathbf{g}) \mathrm{n}=3$ mice/genotype. HeF, heterozygous floxed Srf; HoF, homozygous floxed Srf. n.s., not significant.

\section{Supplementary Fig. 11, Normal body weight and kidney in tamoxifen-treated Srff/ffl/Itga8-}

CreER ${ }^{T 2}$ mice. a, Body weights at baseline and 8 weeks post-tamoxifen treatment in heterozygous Srf knockout (HeF; n=3) and homozygous Srf knockout (HoF; n=6) mice. b, Western blot of ITGA8 and SRF protein in aorta of Srfl/fl/Itga8-CreER ${ }^{T 2}$ mice treated with Oil or tamoxifen (Tmx) 6 weeks after initial dosing. c, H\&E staining of kidney in HeF versus HoF mice 6 weeks after Tmx administration. Scale bar is $50 \mu \mathrm{m}$. d, Coomassie-stained acrylamide gel of urine samples from male $(\mathrm{m})$ and female $(\mathrm{f})$ mice of indicated Itga8-CreER ${ }^{T 2}$-mediated floxed Srf knockout genotypes. Bovine serum albumin (BSA) was used as a control for visualizing evidence of proteinuria. 
Figure 1
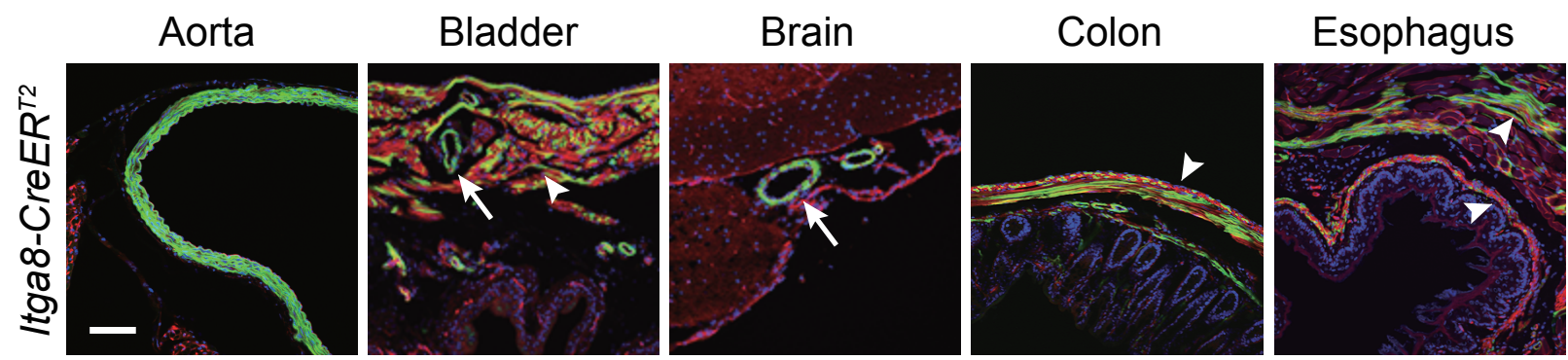

Intestine
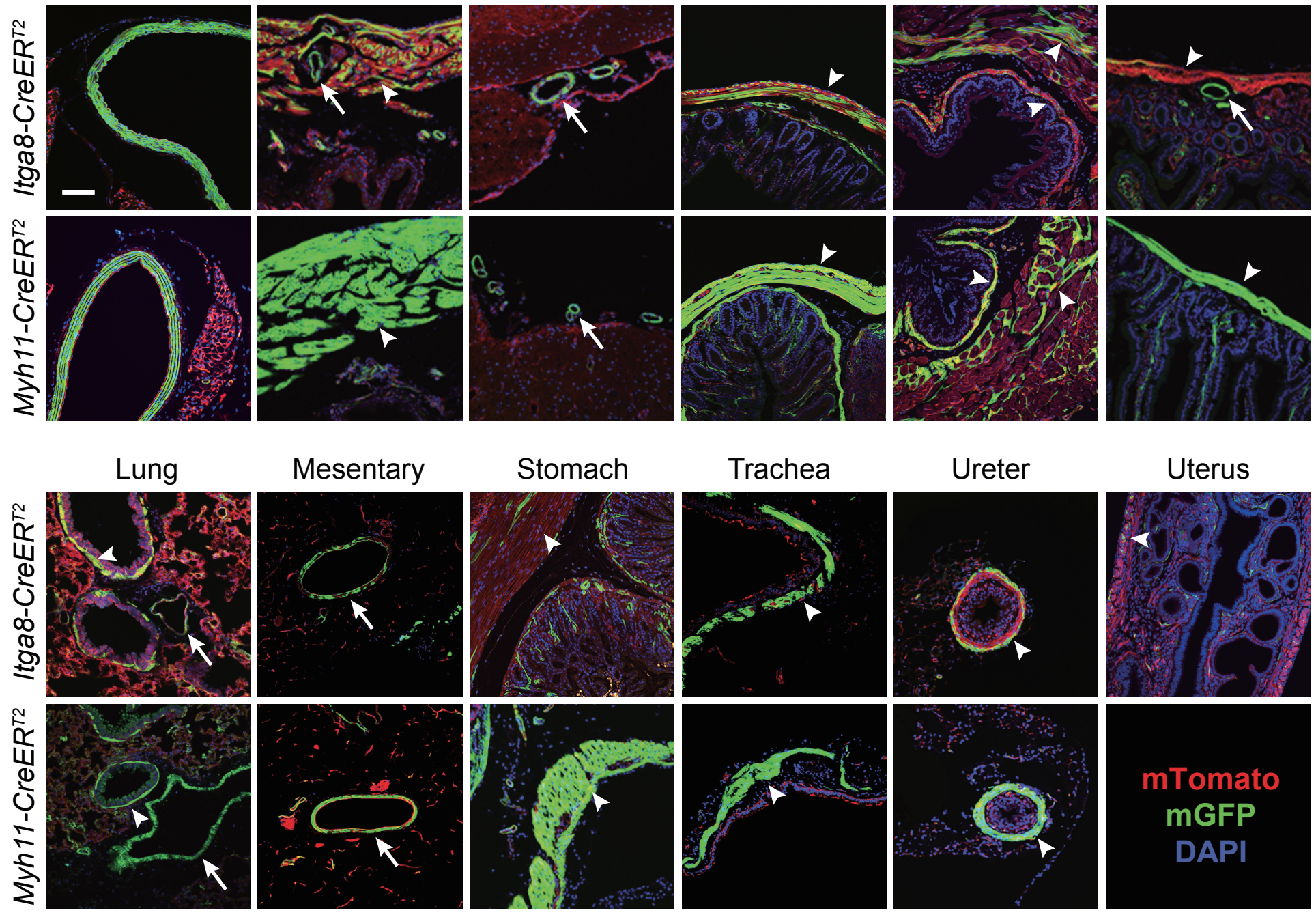

mTomato MGFP

DAPI

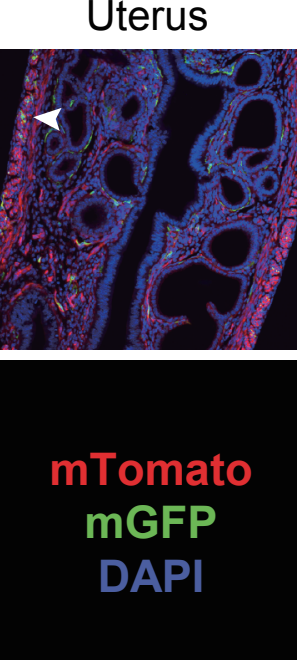


a

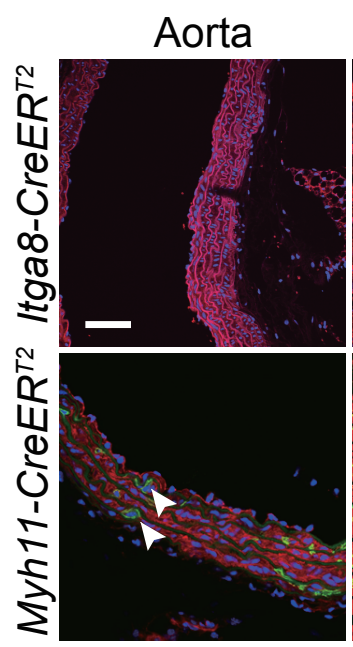

Bladder
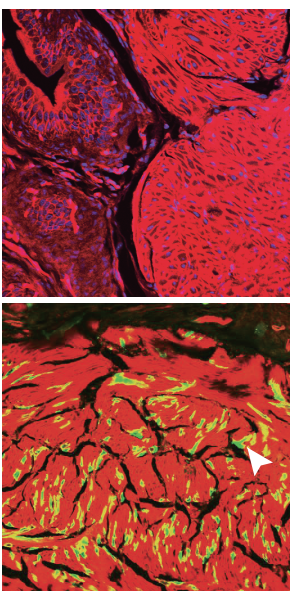

\section{Esophagus}
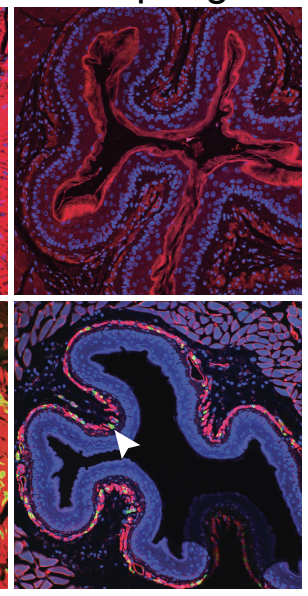

Intestine

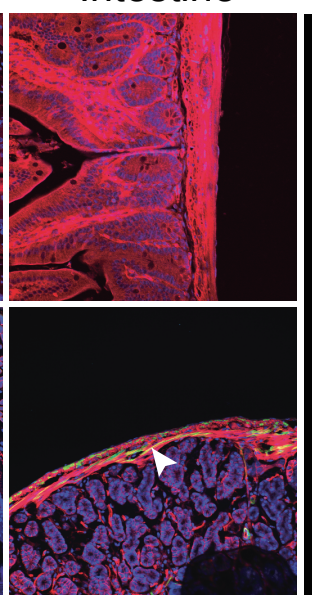

C
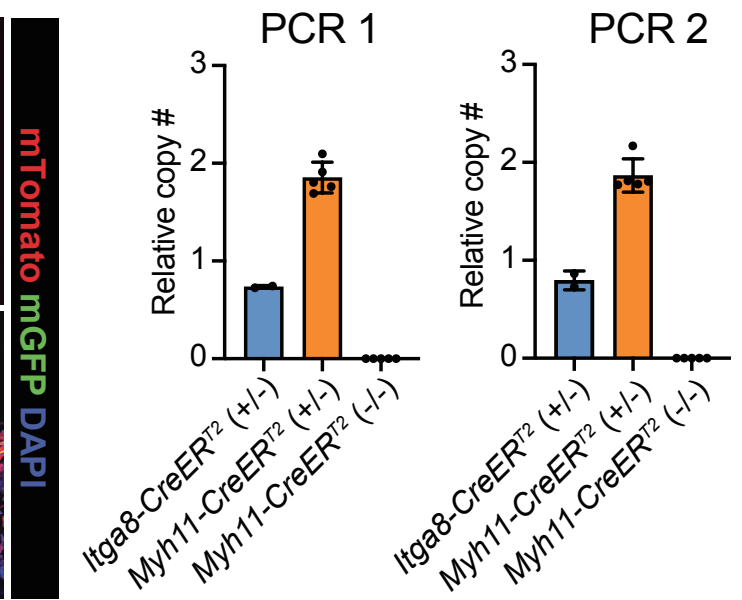

d
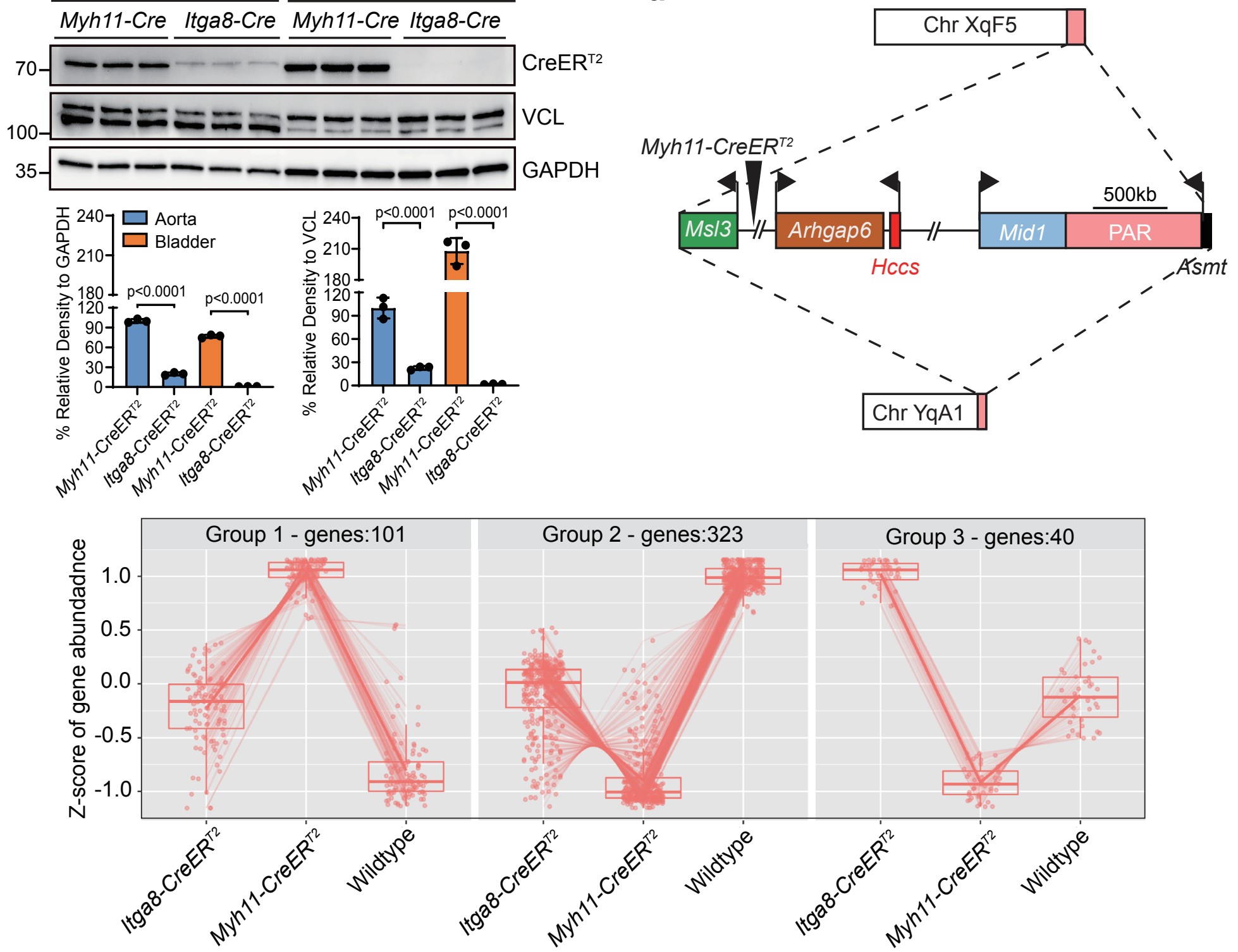
a

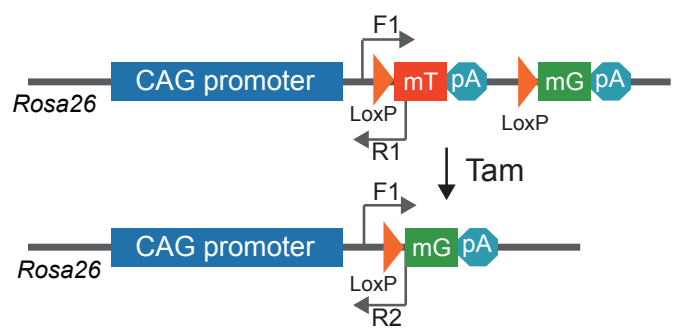

b

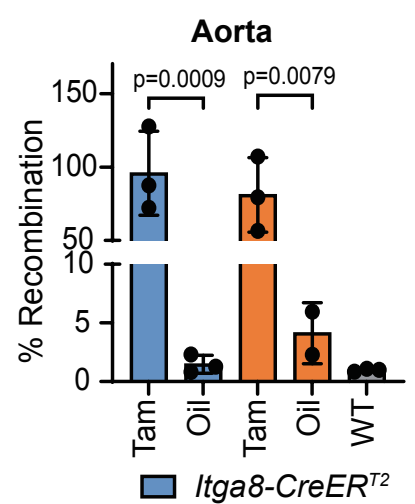

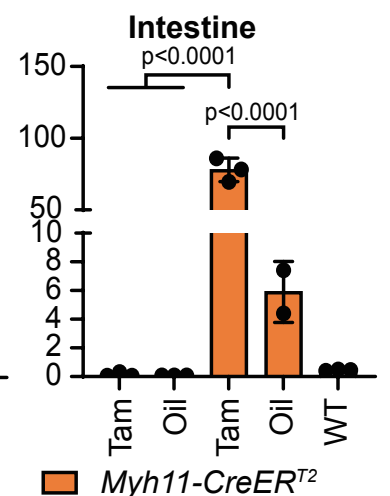

Myh11-CreER ${ }^{\text {T2 }}$

C

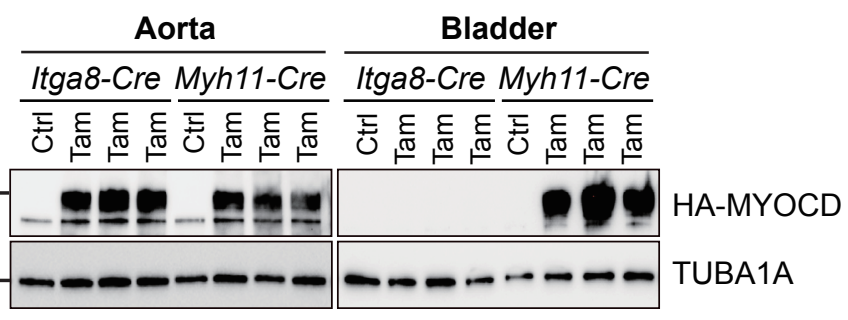

d
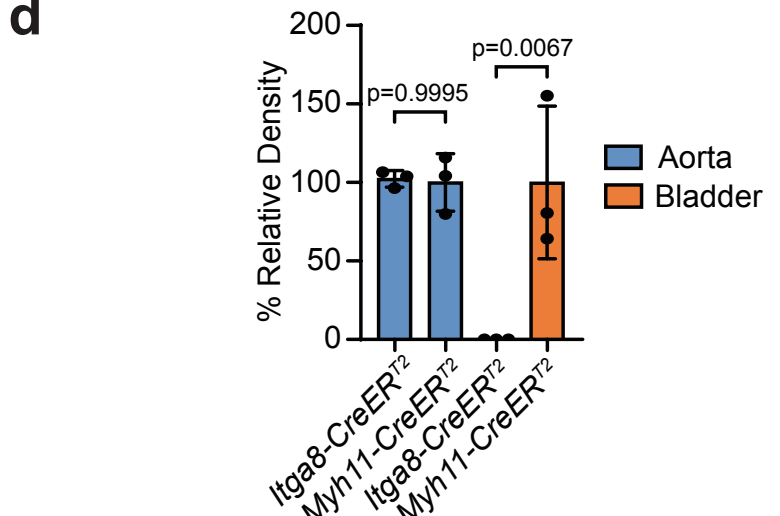

g

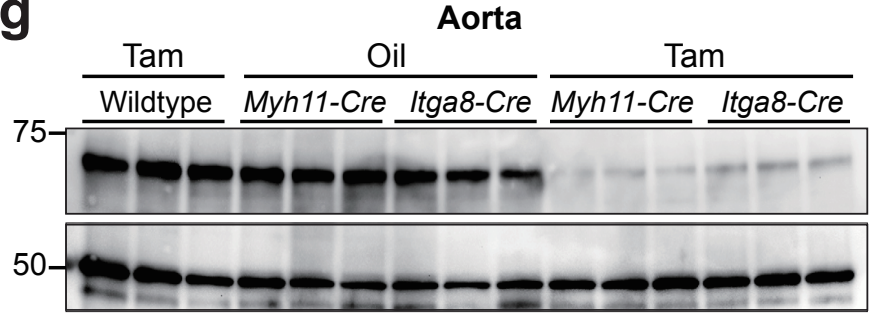

Bladder

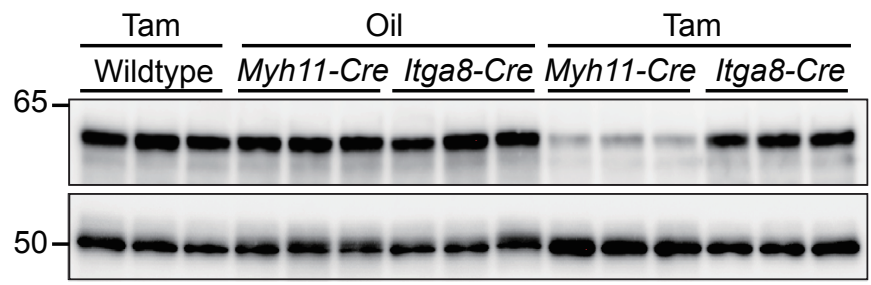

Tam

\section{SRF}

TUBA1A

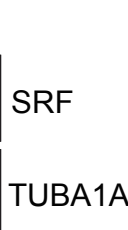

e

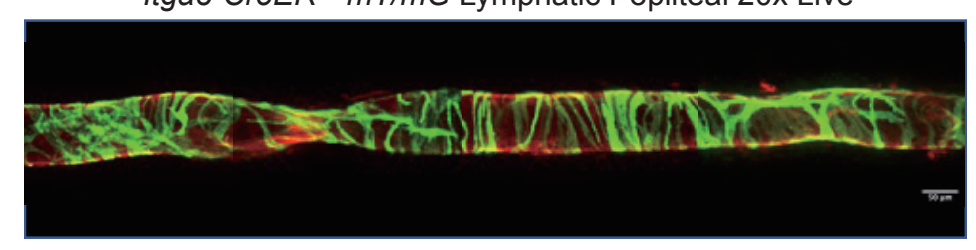

Myh11-CreER ${ }^{T 2} m T / m G$ Lymphatic Popliteal 20x Live

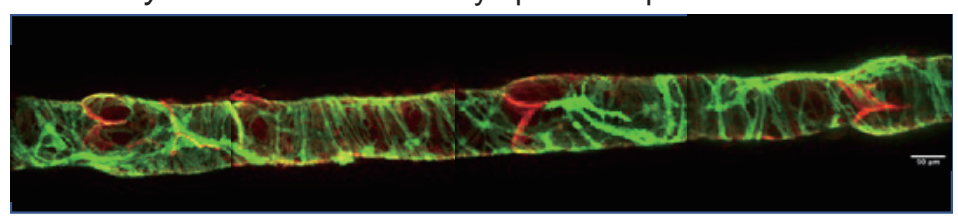

Itga8-CreER ${ }^{T 2} \mathrm{mT} / \mathrm{mG}$ Lymphatic Popliteal 40x Stained

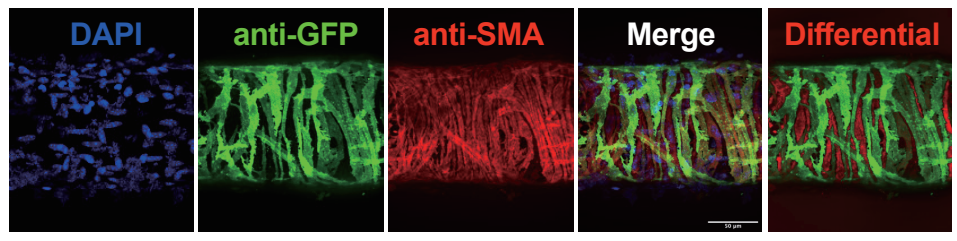

Myh11-CreER ${ }^{T 2} \mathrm{mT} / \mathrm{mG}$ Lymphatic Popliteal 40x Stained

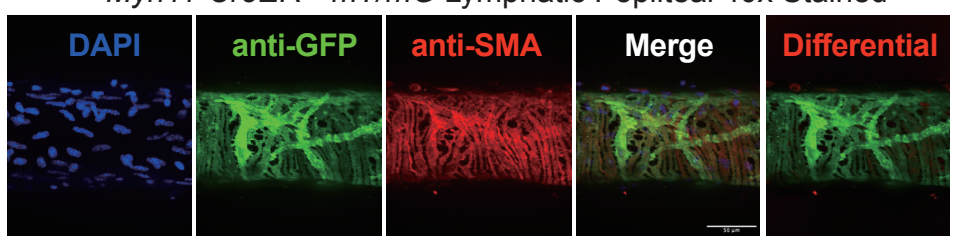

f
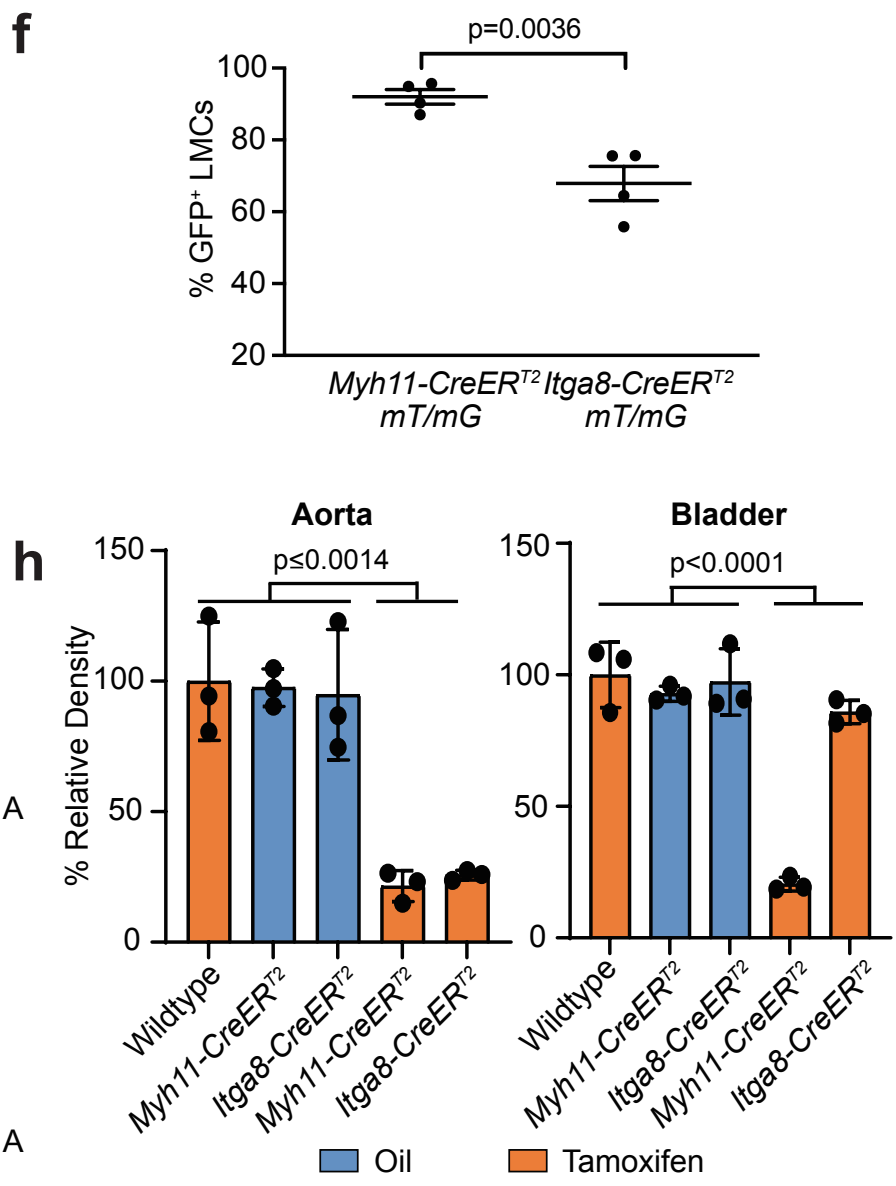
a

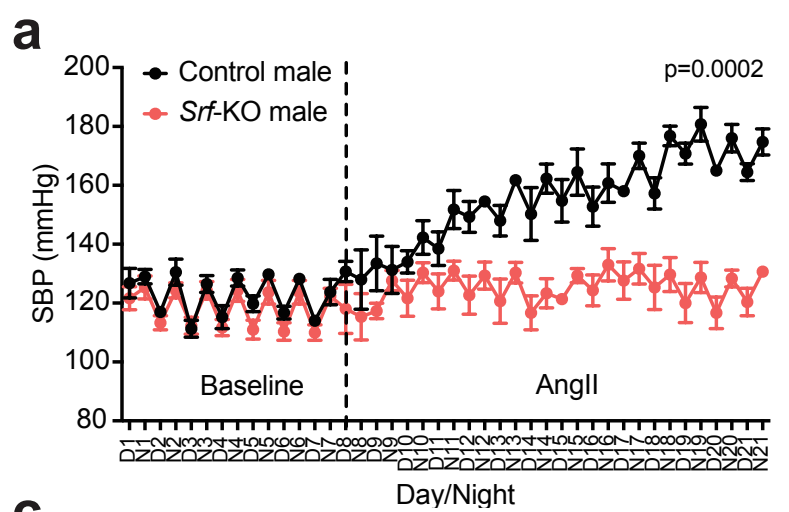

C
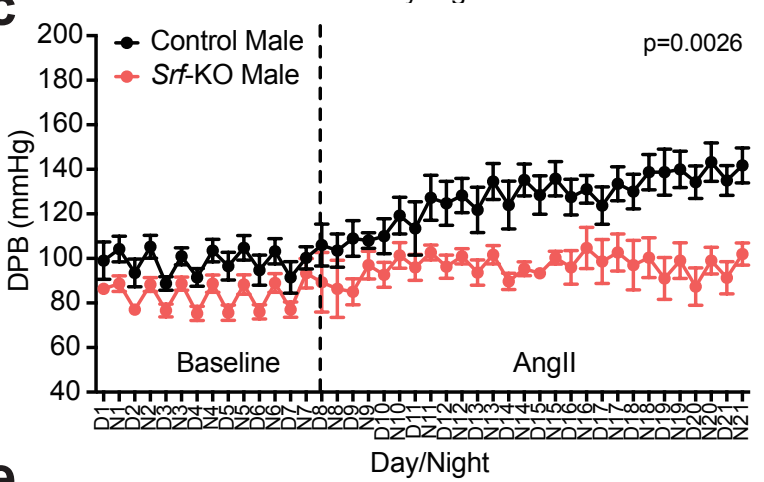

e

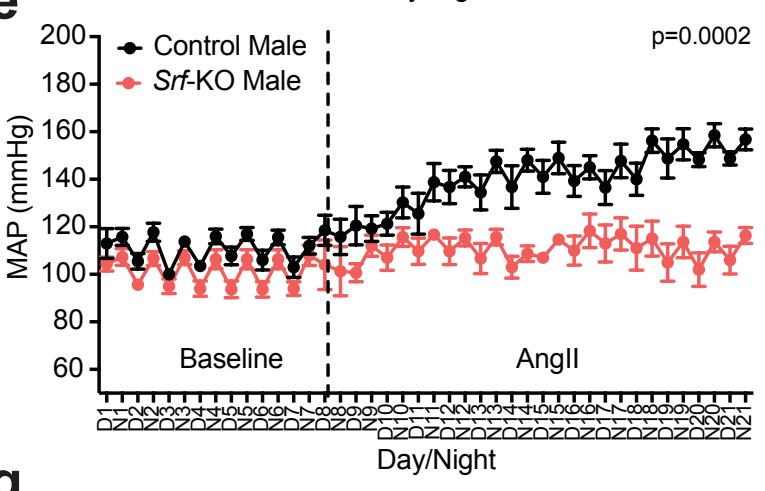

g

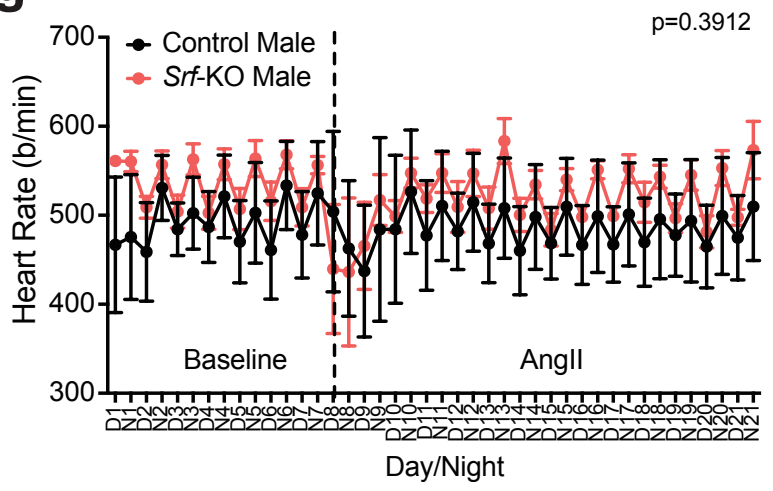

b
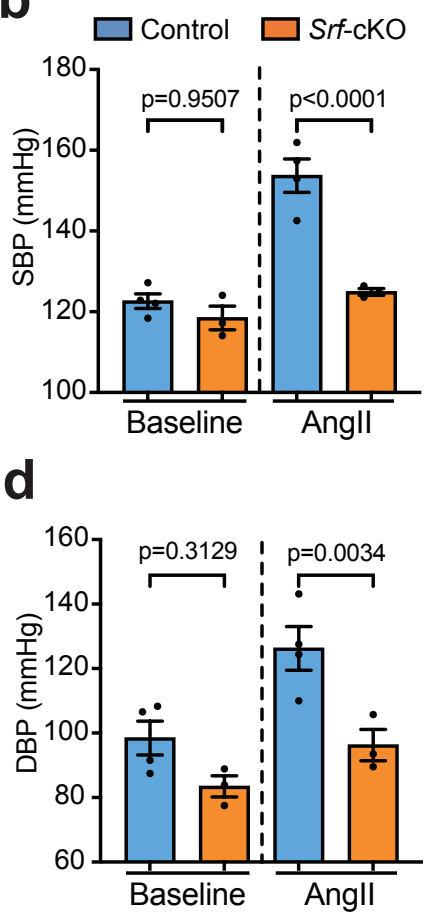

f

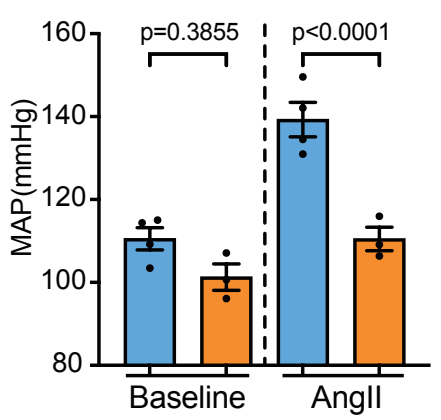

h

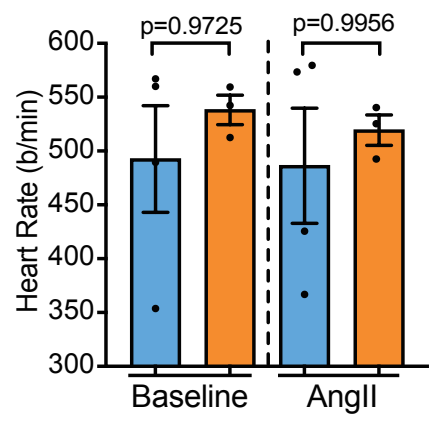


a

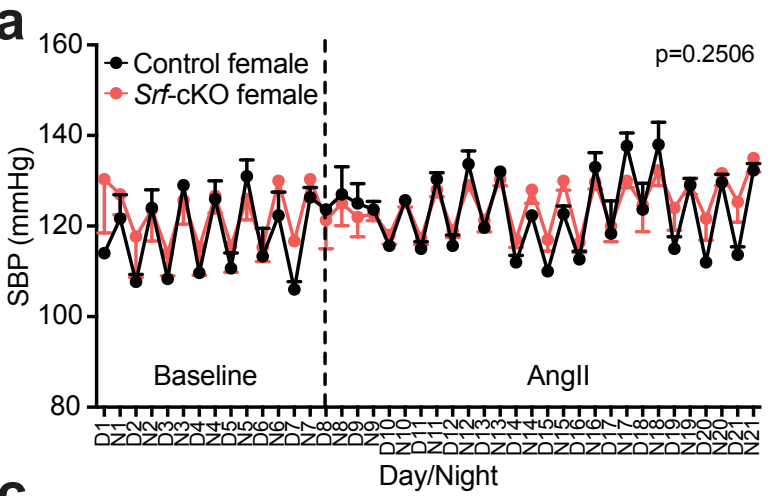

C

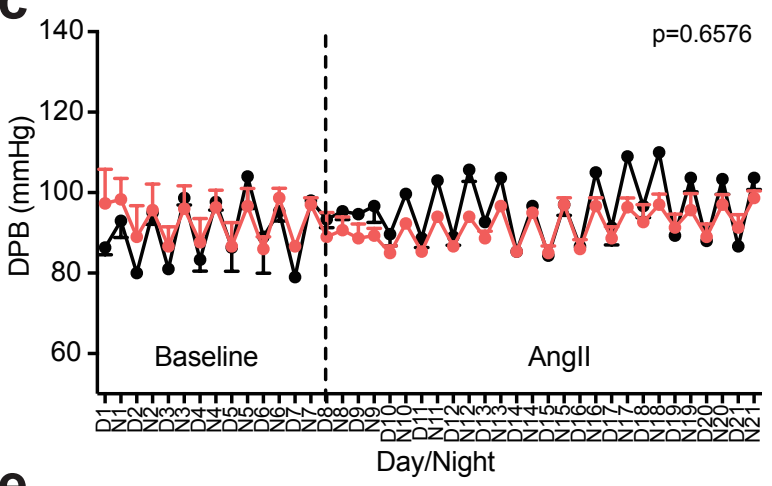

e
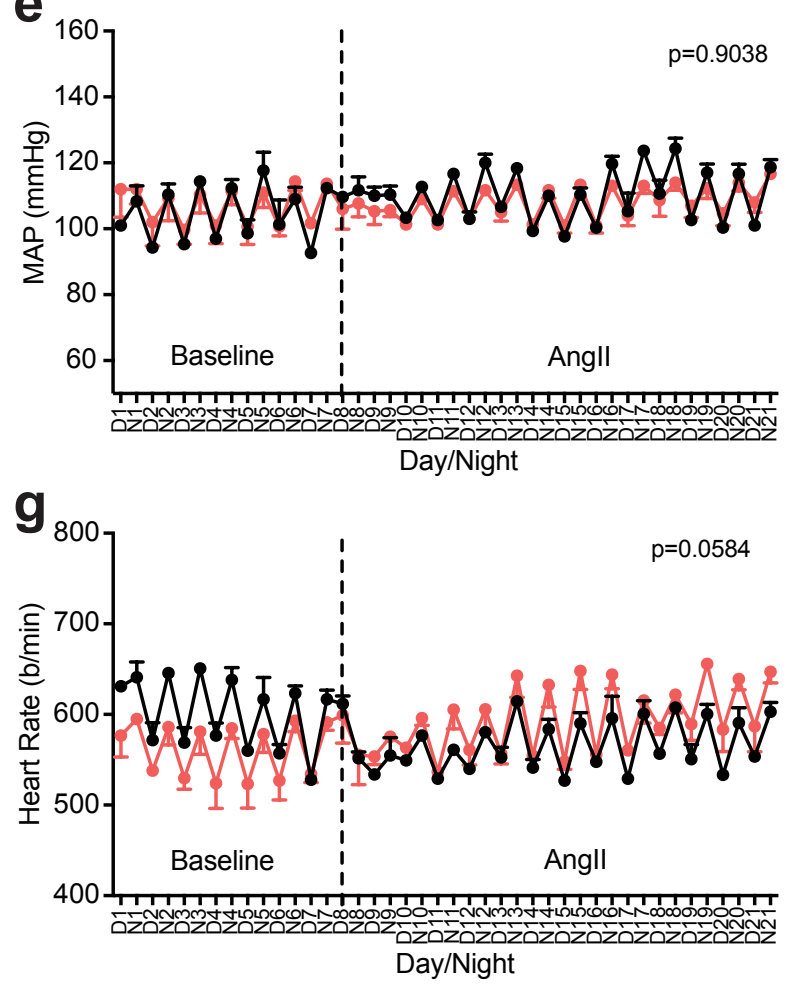

b
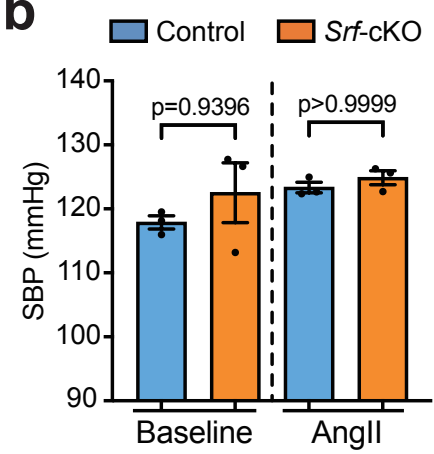

d

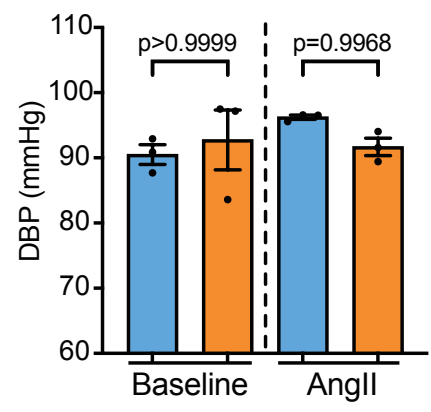

f

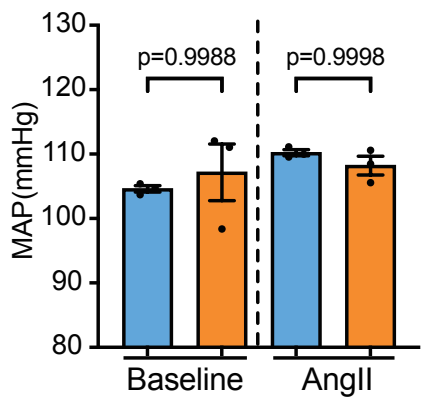

h

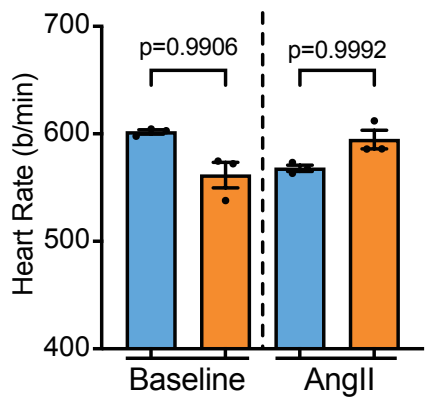


a

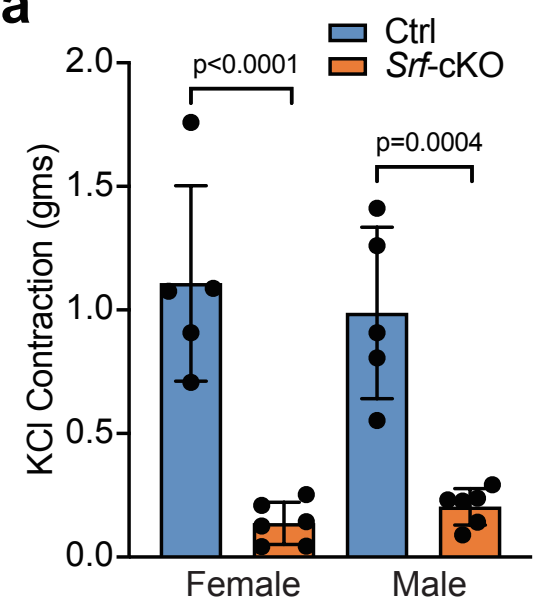

d

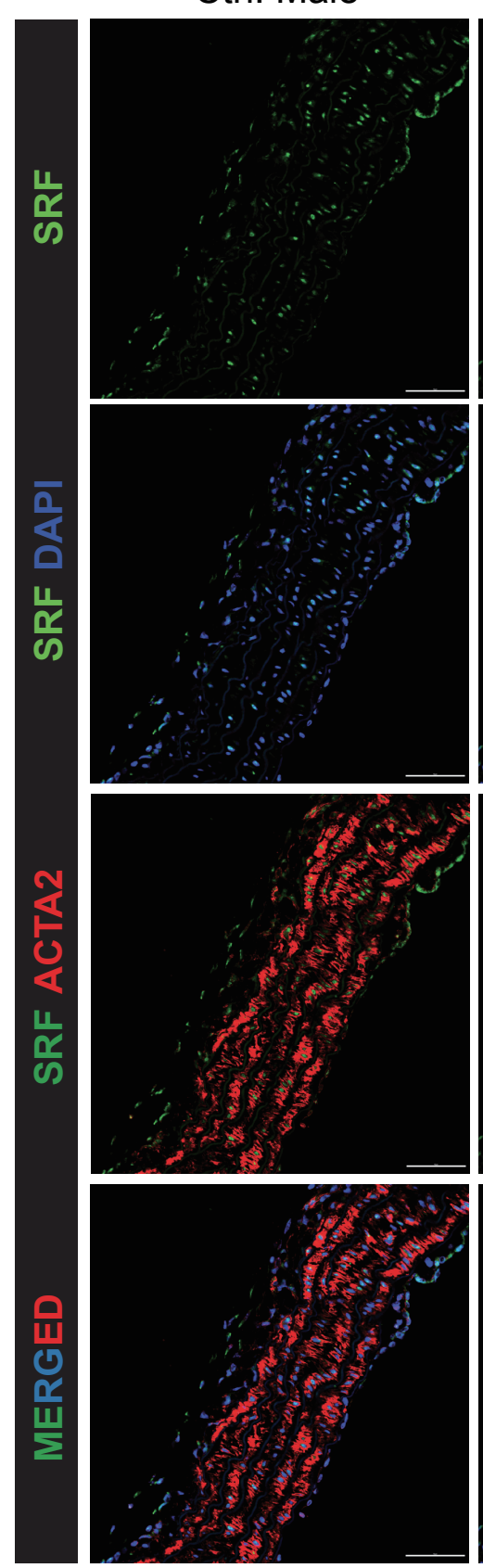

b

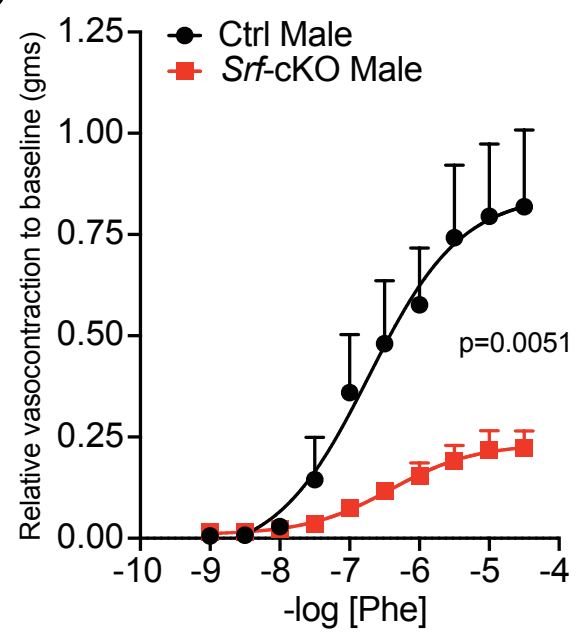

Srf-cKO Male
C

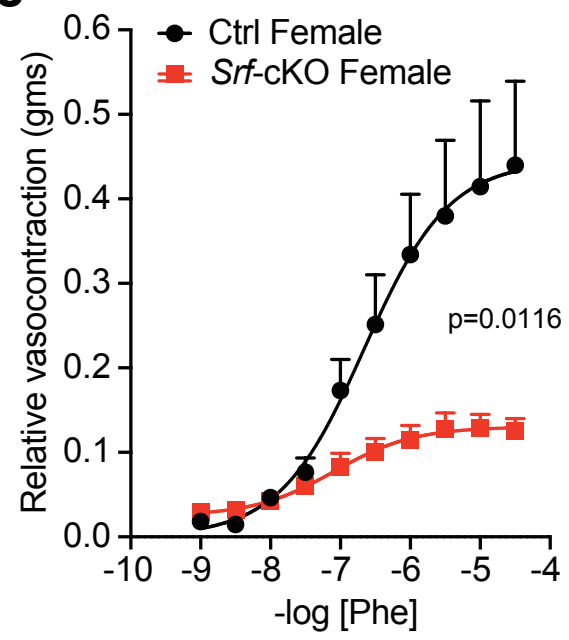

Ctrl. Female

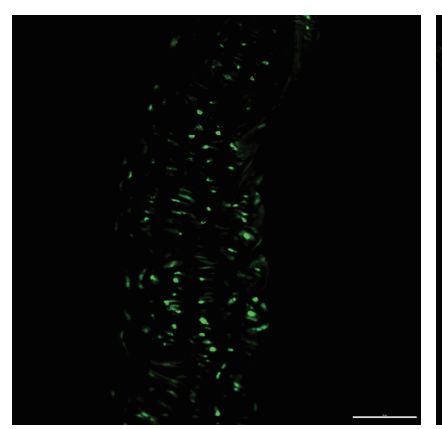

Srf-cKO Female

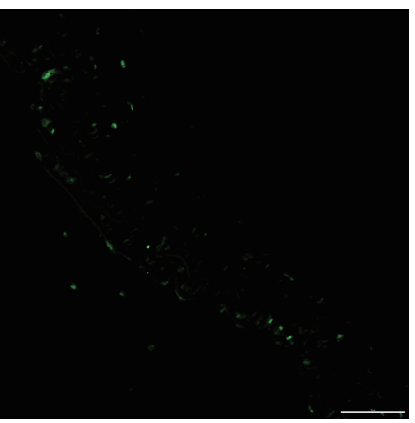

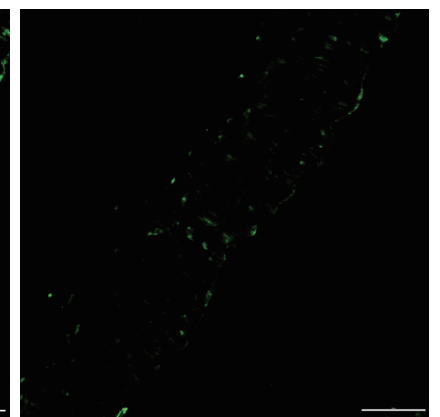
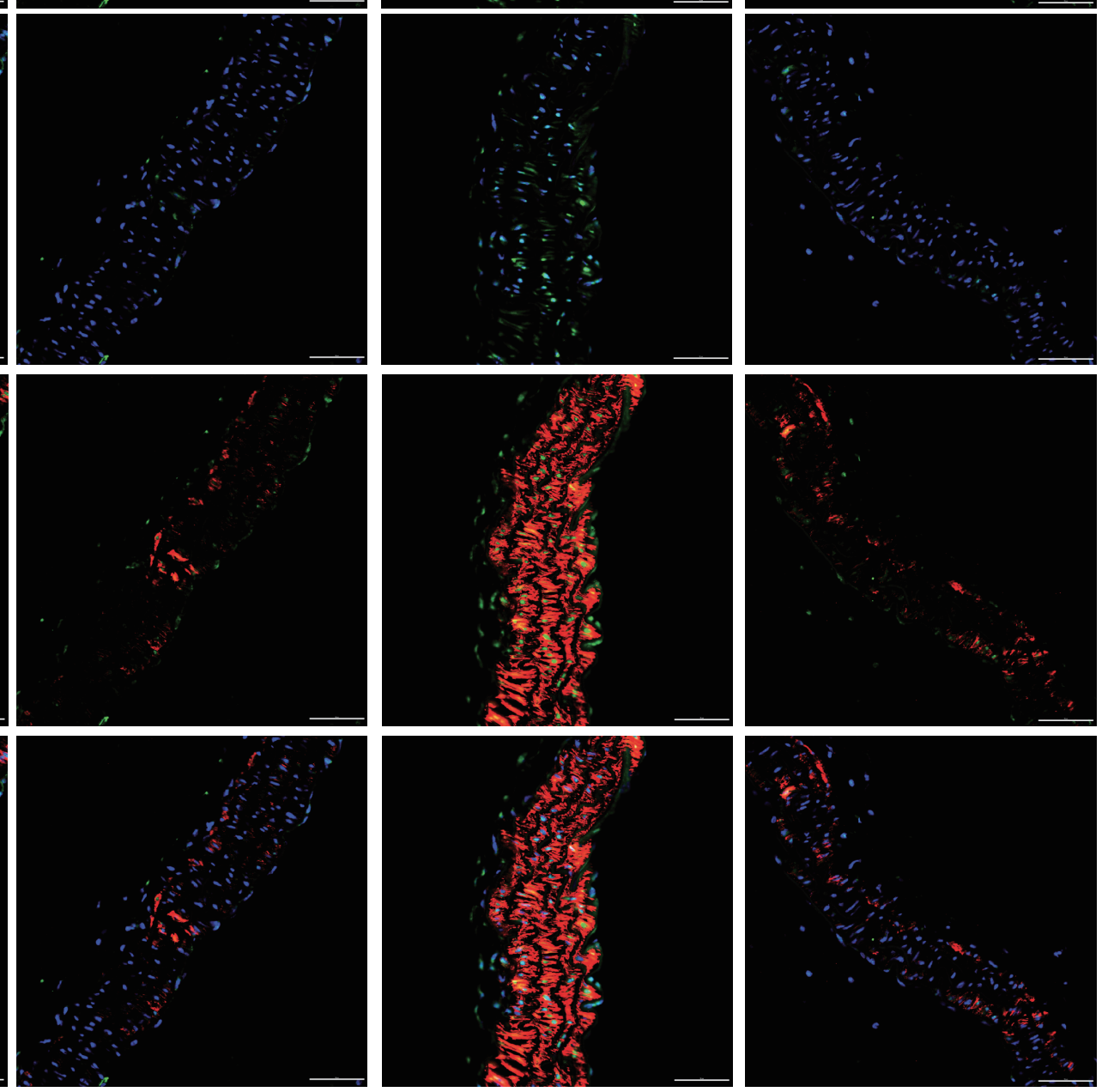
bioRxiv preprint doi: https://doi.org/10.1101/2022.02.03.479061; this version posted February 4, 2022. The copyright holder for this preprint (which was not certified by peer review) is the author/funder. All rights reserved. No reuse allowed without permission.

Figure 7

a

$\begin{array}{ccccc}\text { Gene } & \text { Heterozygous } & \text { Homozygous } & \text { log2FC } & \text { q value } \\ \text { Myh11 } & 274,498 & 160,741 & -0.743 & 1.39 \mathrm{E}-11 \\ \text { Cnn1 } & 26,255 & 14,016 & -0.858 & 2.65 \mathrm{E}-11 \\ \text { Csrp1 } & 35,644 & 21,858 & -0.683 & 2.65 \mathrm{E}-11 \\ \text { Flna } & 562,420 & 334,133 & -0.721 & 3.06 \mathrm{E}-10 \\ \text { Tagln } & 93,661 & 52,342 & -0.797 & 3.06 \mathrm{E}-10 \\ \text { Kcnmb1 } & 3,803 & 2,289 & -0.704 & 6.94 \mathrm{E}-10 \\ \text { Acta2 } & 236,560 & 126,950 & -0.845 & 7.60 \mathrm{E}-10 \\ \text { Mylk } & 55,146 & 35,710 & -0.606 & 8.07 \mathrm{E}-09 \\ \text { Myl9 } & 54,822 & 31,349 & -0.759 & 6.29 \mathrm{E}-08 \\ \text { Actg2 } & 4,715 & 2,451 & -0.865 & 1.46 \mathrm{E}-07 \\ \text { Tspan2 } & 7,220 & 4,063 & -0.762 & 4.84 \mathrm{E}-06 \\ \text { Tgfb1i1 } & 6,506 & 4,050 & -0.635 & 8.11 \mathrm{E}-05 \\ \text { Lmod1 } & 19,243 & 12,486 & -0.581 & 3.43 \mathrm{E}-04 \\ \text { Actb } & 105,442 & 70,375 & -0.542 & 1.30 \mathrm{E}-03 \\ \text { Srf } & 1,897 & 1,262 & -0.539 & 3.73 \mathrm{E}-03\end{array}$

b

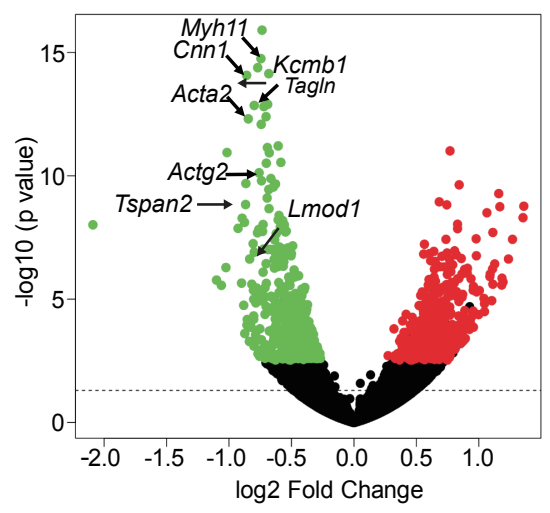

C

\begin{tabular}{llll}
\multicolumn{1}{c}{ TF } & JASPAR ID & \multicolumn{1}{c}{ Family } & Z-score \\
\hline SRF & $\underline{\text { MA0083.1 }}$ & MADS & 26.131 \\
NR1H2-RXRA & $\underline{\text { MA0115.1 }}$ & $\begin{array}{l}\text { Hormone-nuclear Receptor } \\
\text { Homeodomain-NFI-CCAAT- }\end{array}$ & 18.661 \\
TLX1-NFIC & $\underline{\text { MA0119.1 }}$ & $\begin{array}{l}\text { Homding } \\
\text { bind }\end{array}$ & 16.998 \\
MEF2A & $\underline{\text { MA0052.1 }}$ & MADS & 15.596 \\
TBP & $\underline{\text { MA0108.2 }}$ & TATA-binding & 14.357 \\
CEBPA & $\underline{\text { MA0102.2 }}$ & Leucine Zipper & 10.934 \\
TEAD1 & $\underline{\text { MA0090.1 }}$ & Homeodomain & 10.629 \\
SPIB & $\underline{\text { MA0081.1 }}$ & Ets & 9.205 \\
Hand1-Tcfe2a & $\underline{\text { MA0092.1 }}$ & Helix-Loop-Helix & 8.952 \\
PPARG-RXRA & $\underline{\text { MA0065.2 }}$ & Hormone-nuclear Receptor & 8.787
\end{tabular}

d

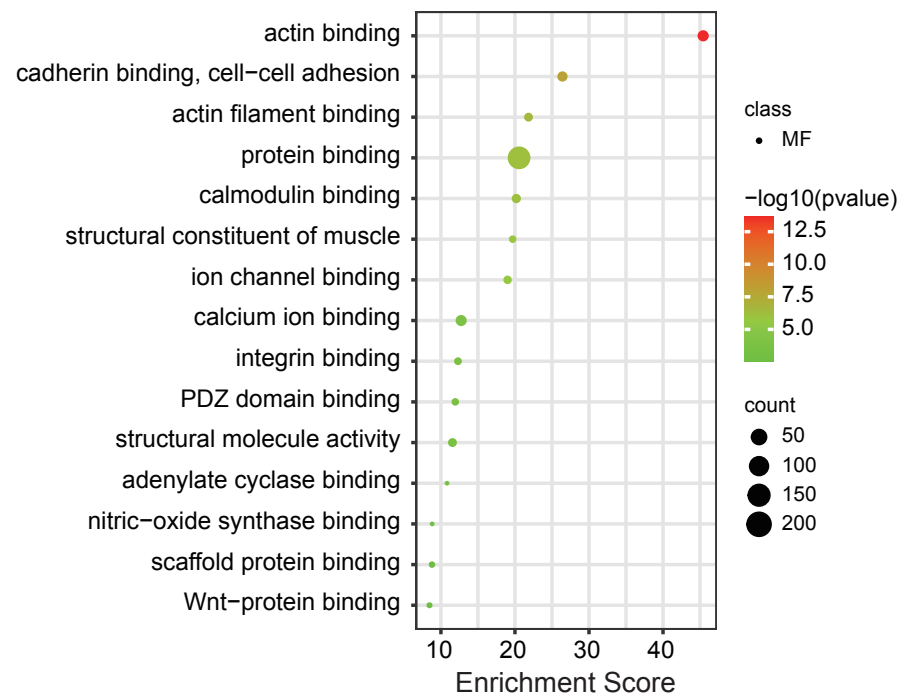

e

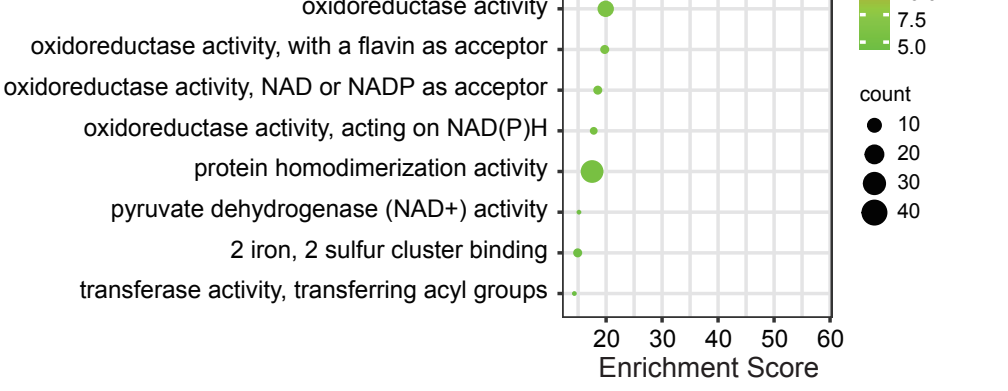



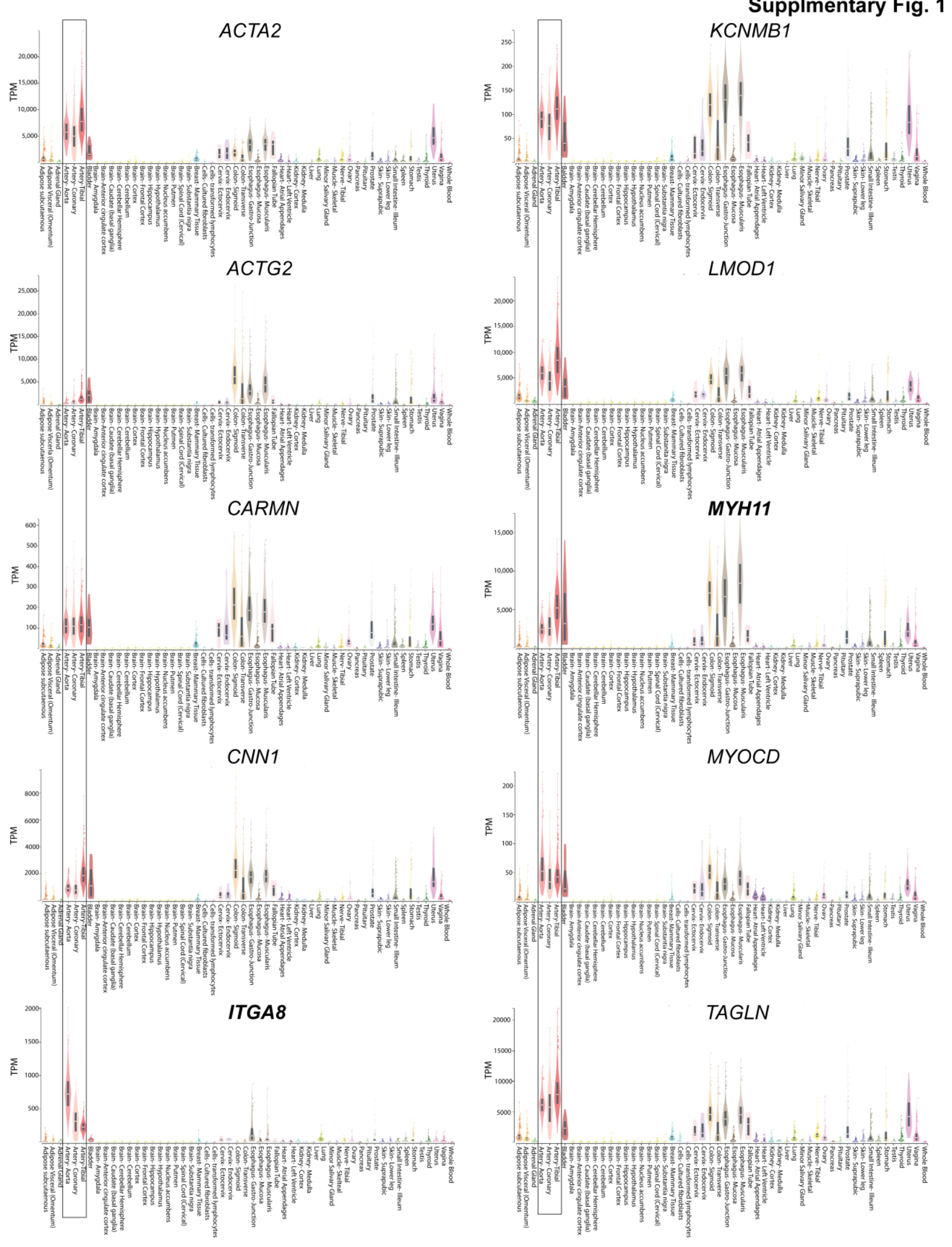


\section{Supplementary Fig. 2}

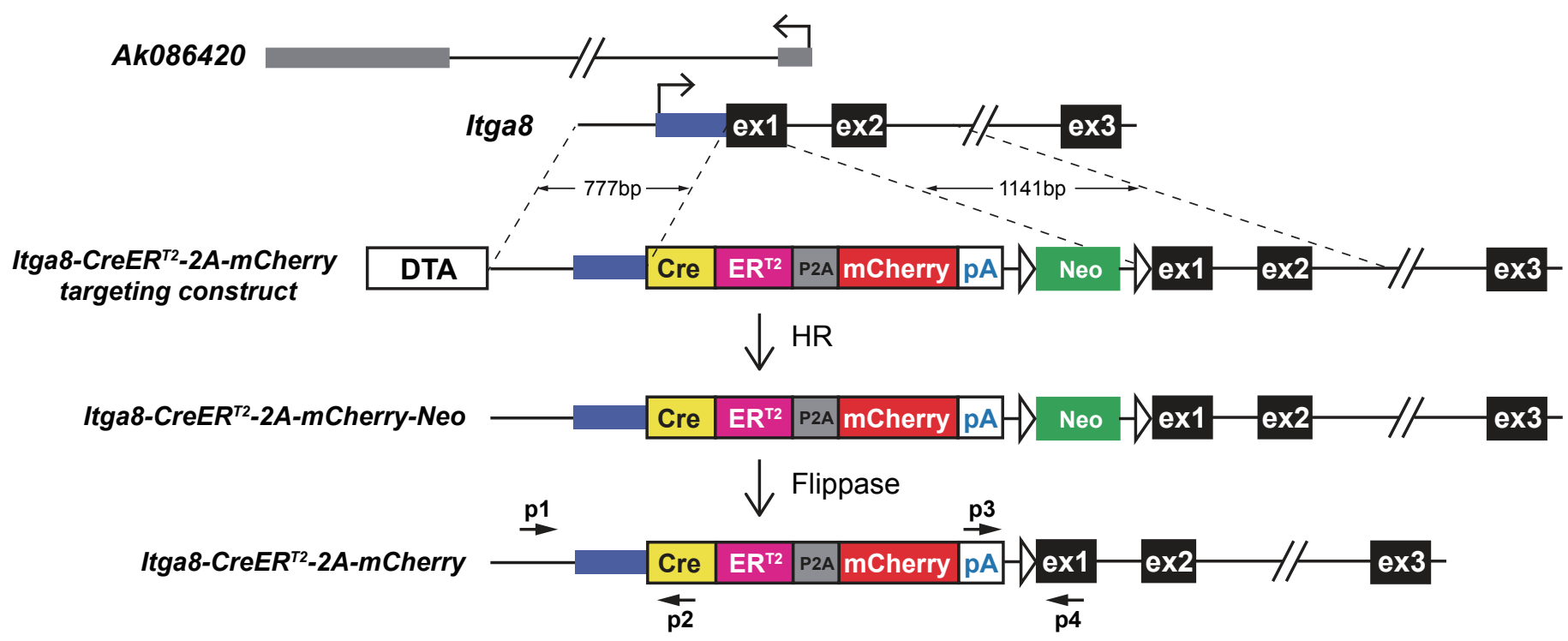

GACTCGAAATCTAGCGGGCTCAACAGGCACTTGCCCGGTCCTAGACCGCAAGCTCCGTCCTGCAGCCTGAGAACTAGCCGCTAGAGGGCGCGAAGAGCGCGCCGGCC CCAGCCAGGGGCTGGAGAGGGTCGAGCGCGGGATCCGCTGCTGCAAGCCCAGAGCAGCGGGGTCGGCGGGCCGCACAGGGCGAGAGGACTGTCACTGTGGACGCTCC TAGCTCACCGACCCGGGACACCGGCCTCTGGGGAGCGTCAGGCCTTCTCCTCTGGGCTCCACGGCTCCCGATCCCGGGTGAACAGCGTTCGGGAAGGAGGGCGGCCG CCACCATGTCCAATTTACTGACCGTACACCAAAATTTGCCTGCATTGCCGGTCGATGCAACGAGTGATGAGGTTCGCAAGAACCTGATGGACATGTTCAGGGATCGC CAGGCGTTTTCTGAGCATACCTGGAAAATGCTTCTGTCCGTTTGCCGGTCGTGGGCGGCATGGTGCAAGTTGAATAACCGGAAATGGTTTCCCGCAGAACCTGAAGA TGTTCGCGATTATCTTCTATATCTTCAGGCGCGCGGTCTGGCAGTAAAAACTATCCAGCAACATTTGGGCCAGCTAAACATGCTTCATCGTCGGTCCGGGCTGCCAC GACCAAGTGACAGCAATGCTGTTTCACTGGTTATGCGGCGCATCCGAAAAGAAAACGTTGATGCCGGTGAACGTGCAAAACAGGCTCTAGCGTTCGAACGCACTGAT TTCGACCAGGTTCGTTCACTCATGGAAAATAGCGACCGCTGCCAGGATATACGTAATCTGGCATTTCTGGGGATTGCTTATAACACCCTGTTACGTATAGCCGAAAT TGCCAGGATCAGGGTTAAAGACATCTCACGTACTGACGGTGGGAGAATGTTAATCCATATTGGCAGAACGAAAACGCTGGTTAGCACCGCAGGTGTAGAGAAGGCAC TTAGCCTGGGGGTAACTAAACTGGTCGAGCGATGGATTTCCGTCTCTGGTGTAGCTGATGATCCGAATAACTACCTGTTTTGCCGGGTCAGAAAAAATGGTGTTGCC GCGCCATCTGCCACCAGCCAGCTATCAACTCGCGCCCTGGAAGGGATTTTTGAAGCAACTCATCGGTTGATTTACGGCGCTAAGGATGACTCTGGTCAGAGATACCT GGCCTGGTCTGGACACAGTGCCCGTGTCGGAGCCGCGCGAGATATGGCCCGCGCTGGAGTTTCAATACCGGAGATCATGCAAGCTGGTGGCTGGACCAATGTAAATA TTGTCATGAACTATATCCGTAACCTGGATAGTGAAACAGGGGCAATGGTGCGCCTGCTGGAAGATGGCGATCTgGAGCCATCTGCTGGAGACATGAGAGCTGCCAAC CTTTGGCCAAGCCCGCTCATGATCAAACGCTCTAAGAAGAACAGCCTGGCCTTGTCCCTGACGGCCGACCAGATGGTCAGTGCCTTGTTGGATGCT GAGCCCCCCAT ACTCTATTCCGAGTATGATCCTACCAGACCCTTCAGTGAgGCTTCGATGATGGGCTTACTGACCAACCTGGCAGACAGGGAGCTGGTTCACATGATCAACTGGGCGA AGAGGGTGCCAGGCTTTGTGGATTTGACCCTCCATGATCAGGTCCACCTTCT GGAATGTGCCTGGCTAGAGATCCT GATGATTGGTCTCGTCTGGCGCTC TATGGAG CACCCAGTGAAGCTACTGTTTGCTCCTAACTTGCTCTTGGACAGGAACCAGGGAAAATGTGTAGAGGGCATGGTGGAaATCTTCGACATGCTGCTGGCTACATCATC TCGGTTCCGCATGATGAATCTGCAGGGAGAGGAGTTTGTGTGCCTCAAATCTATTATTTTGCTTAATTCTGGAGTGTACACATTTCTGTCCAGCACCCTGAAGTCTC TGGAAGAGAAGGACCATATCCACCGAGTCCTGGACAAGATCACAGACACTTTGATCCACCTGATGGCCAAGGCAGGCCTGACCCTGCAGCAGCAGCACCAGCGGCTG GCCCAGCTCCTCCTCATCCTCTCCCACATCAGGCACATGAGTAACAAAGGCATGGAGCATCTGTACAGCATGAAGTGCAAGAACGTGGTGCCCCTCTATGACCTGCT GCTGGAGGCGGCGGACGCCCACCGCCTACATGCGCCCACTAGCCGTGGAGGGGCATCCGTGGAGGAGACGGACCAAAGCCACTTGGCCACTGCGGGCTCTACTTCAT CGCATTCCTTGCAAAAGTATTACATCACGGGGGAGGCAGAGGGTTTCCCTGCCACAGTCggatccggAggttccggaagcggagctaccaacttcagcctgctgaag caggctggcgacgtggaggagaaccctggacctATGGTTTCAAAAGGTGAAGAAGATAATATGGCTATTATTAAAGAATTTATGAGATTTAAAGTTCATATGGAAGG TTCAGTTAATGGTCATGAATTTGAAATTGAAGGTGAAGGTGAAGGTAGACCATATGAAGGTACTCAAACTGCTAAATTGAAAGTTACTAAAGGTGGTCCATTACCAT TTGCTTGGGATATTTTGTCACCACAATTTATGTATGGTTCAAAAGCaTATGTTAAACATCCAGCTGATATTCCAGATTATTTAAAATTGTCATTTCCAGAAGGTTTT AAATGGGAAAGAGTTATGAATTTTGAAGATGGTGGTGTTGTTACTGTTACTCAAGATTCATCATTACAAGATGGTGAATTTATTTATAAAGTTAAATTGAGAGGTAC TAATTTTCCATCAGATGGTCCAGTTATGCAAAAAAAAACTATGGGTTGGGAgGCTTCATCAGAAAGAATGTATCCAGAAGATGGTGCTTTAAAAGGTGAAATTAAAC AAAGATTGAAATTAAAAGATGGTGGTCATTATGATGCTGAAGTTAAAACTACTTATAAAGCTAAAAAACCAGTTCAATTACCAGGTGCTTATAATGTTAATATTAAA TTGGATATTACTTCACATAATGAAGATTATACTATTGTTGAACAATATGAAAGAGCTGAAGGTAGACATTCAACTGGTGGTATGGATGAATTATATAAATAATAGgg atcCTCTAGACTAGAGCGATCGCGGATCTTTGTGAAGGAACCTACTTCTGTGGTGTGACATAATTGGACAAACTACCTACAGAGATTTAAAGCTCTAAGGTAAATA TAAAATTTTAAGTGTATATGTGTTAAACTACTGTTCTAATTGTTGTGTATTTAAATTCCAACCTATGGAACTGATGAATGGGAGCAGTGGTGGAATGCTTT AATGAGGAAAACCTGTTTTGCTCAGAAGAAATGCCATCTAGTGATGATGAGGCTACTGCTGACTCTCAACATTCTACTCCTCCAAAAAAGAAGAGAAAGGTAGAAGA CCCCAAGGACTTTCCTTCAGAATTGCTAAGTTTTTGAGTCATGCTGTGTTTAGTAATAGAACTCTTGCTTGCTTTGCTATTTACACCACAAAGGAAAAAGCTGCAC TGCTATACAAGAAAATTATGGAAAAATATTCTGTAACCTTTATAAGTAGGCATAACAGTTATAATCATAACATACTGTTTTTTCTTACTCCACACAGGCATAGAGTG TCTGCTATTAATAACTATGCTCAAAAATTGTGTACCTTTAGCTTTTTAATTTGTAAAGGGGTTAATAAGGAATATTTGATGTATAGTGCCTTGACTAGAGATCATAA TCAGCCATACCACATTTGTAGAGGTTTTACTTGCTTTAAAAAACCTCCCACACCTCCCCCTGAACCTGAAACATAAAATGAATGCAATTGTTGTTGTTAACTTGTTT ATTGCAGCTTATAATGGTACAAATAAAGCAATAGCATCACAAATTCACAAATAAAGCATTTTTTCACTGCATTCTAGTTGTGGTTTGCCAAACTCATCAATGT ATCTTATCATGTCTGGATATCGTCGAGCTAGAGCTTGAAGTTCCTATTCTCTAGAAAGTATAGGAACTTCGTCGACCTAGGAATTCGATGTCTGCGGGAACCCACTG TGGTCCCCCGGGGAACCGGGCACCTCCGTTCGCGCGTCTCTGTTGCGTCTCGGCCGCGCTGGGGATGCTGTGGTCCCCTGCGTGTCTGGCGTTCAACTTGGATGTGG ACAAGCTCACTGTGTACAGTGGCCCCGAGGGCAGCTACTTCGGCTACTCACTGGACTTCTACATACCTGATGCGCGCAC

Itga8 5' promoter Itga8 5' UTRKozak consensus sequence CreER ${ }^{\mathrm{T}} \mathrm{P} 2 \mathrm{~A}$

mCherry polyA Itga8 coding sequence 


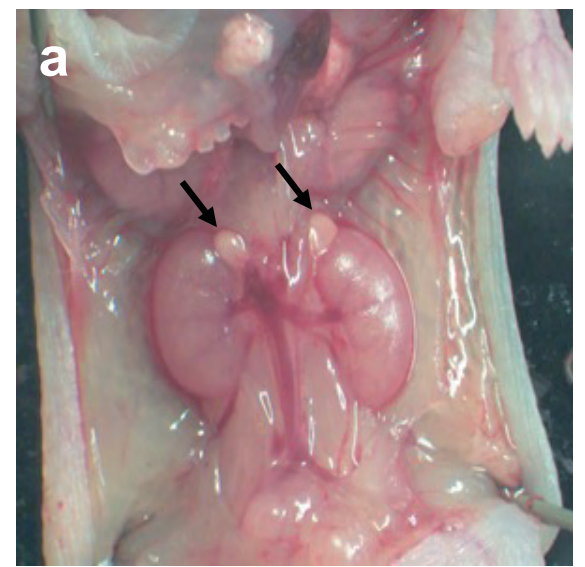

Wild type

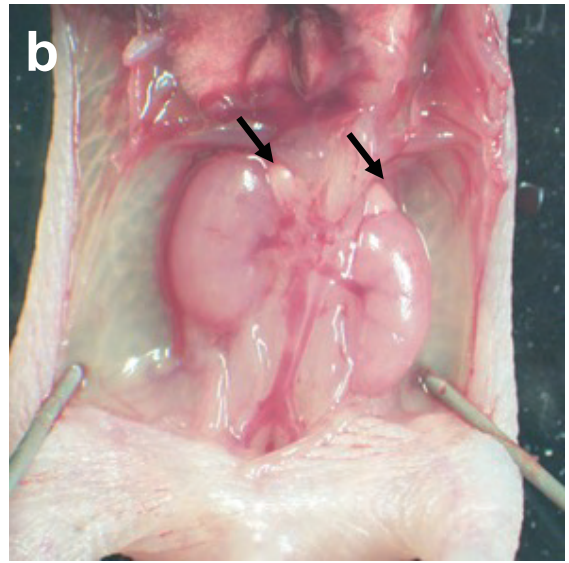

Heterozygous Itga8-CreER ${ }^{T 2}$

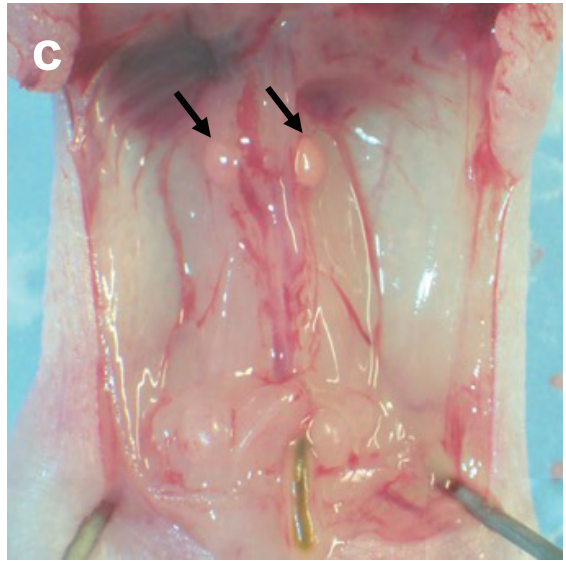

Homozygous Itga8-CreER ${ }^{T 2}$ 
a

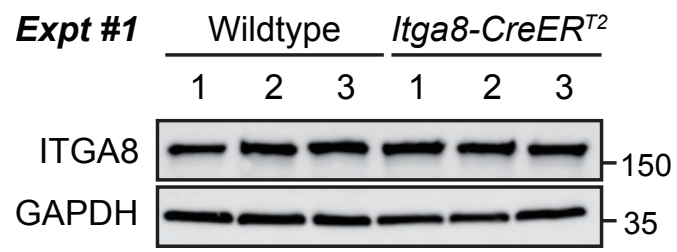

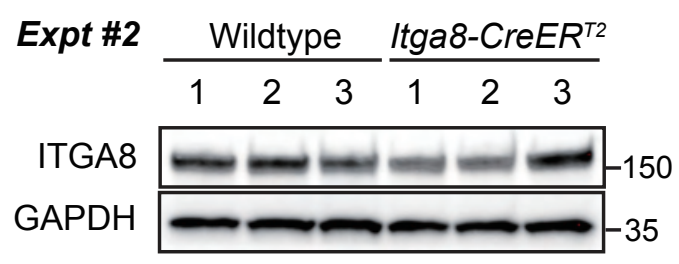

C Ak086420

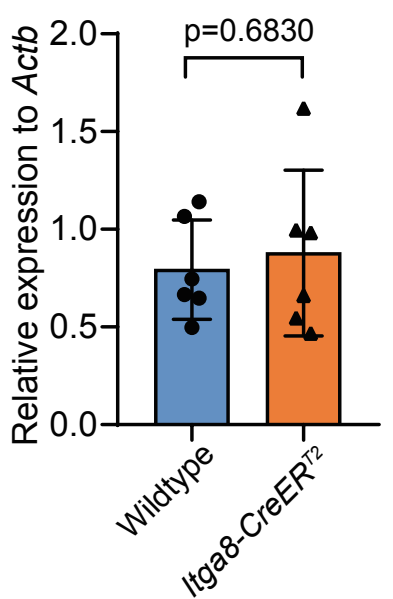

d

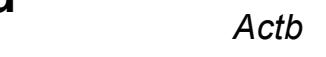

ITGA8

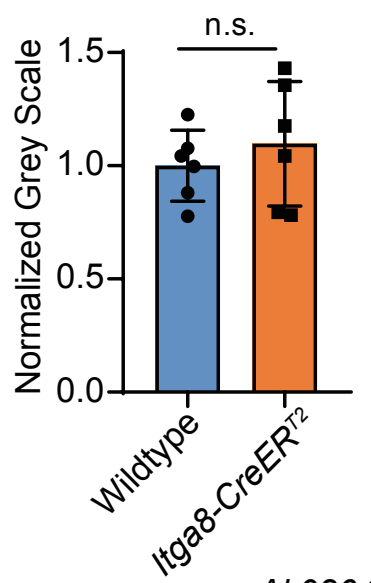

Ak086420

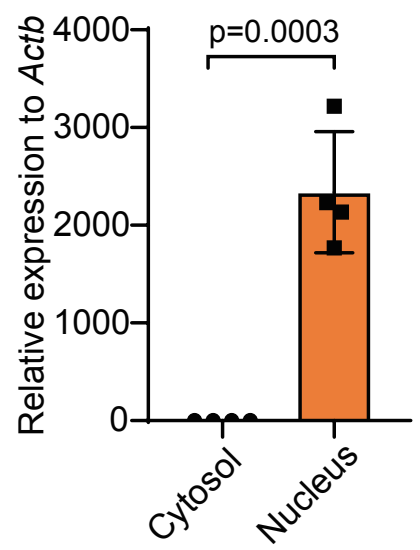

b

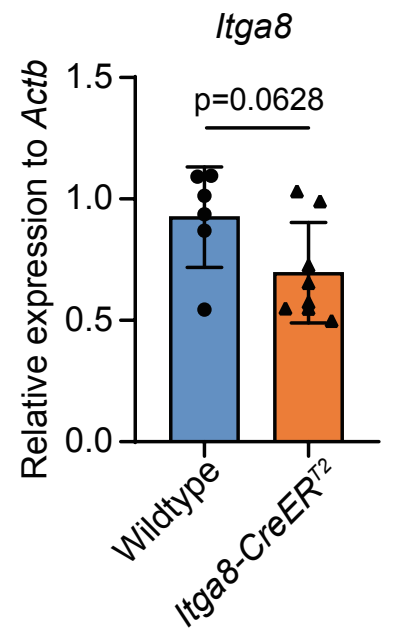

Aorta

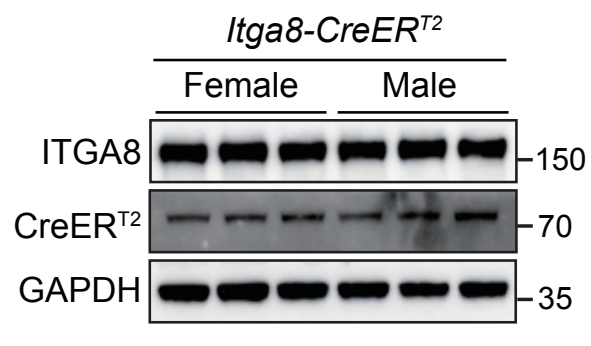


a

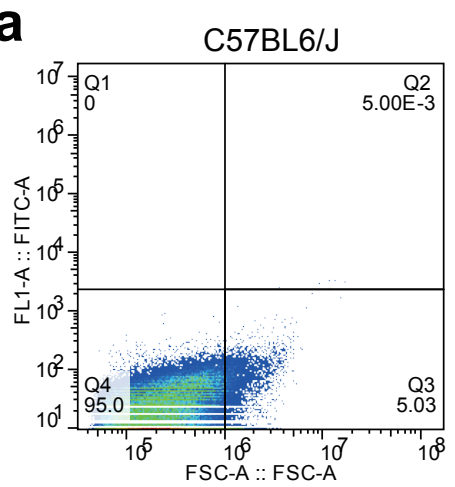

d

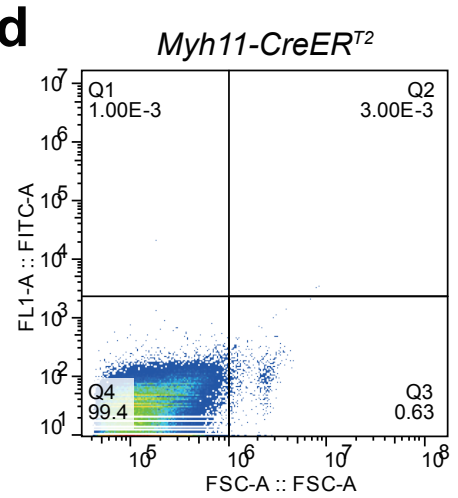

b

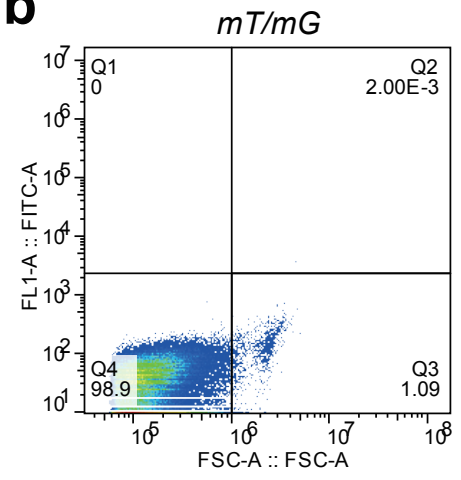

e

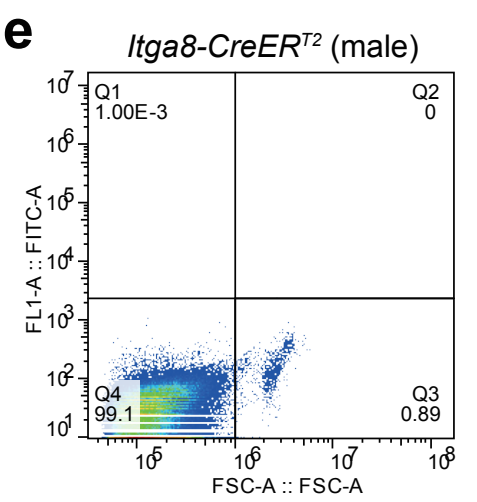

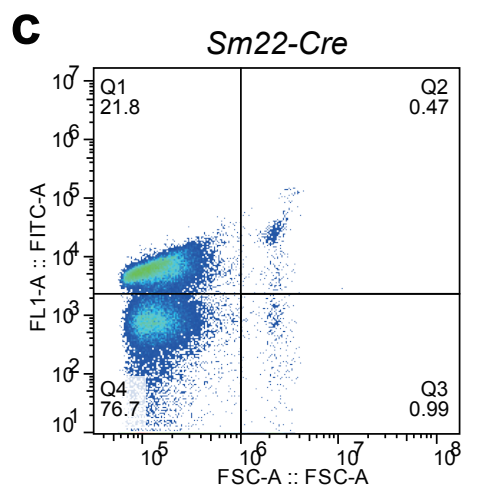

f Itga8-CreER $R^{\text {T2 }}$ (female)

h
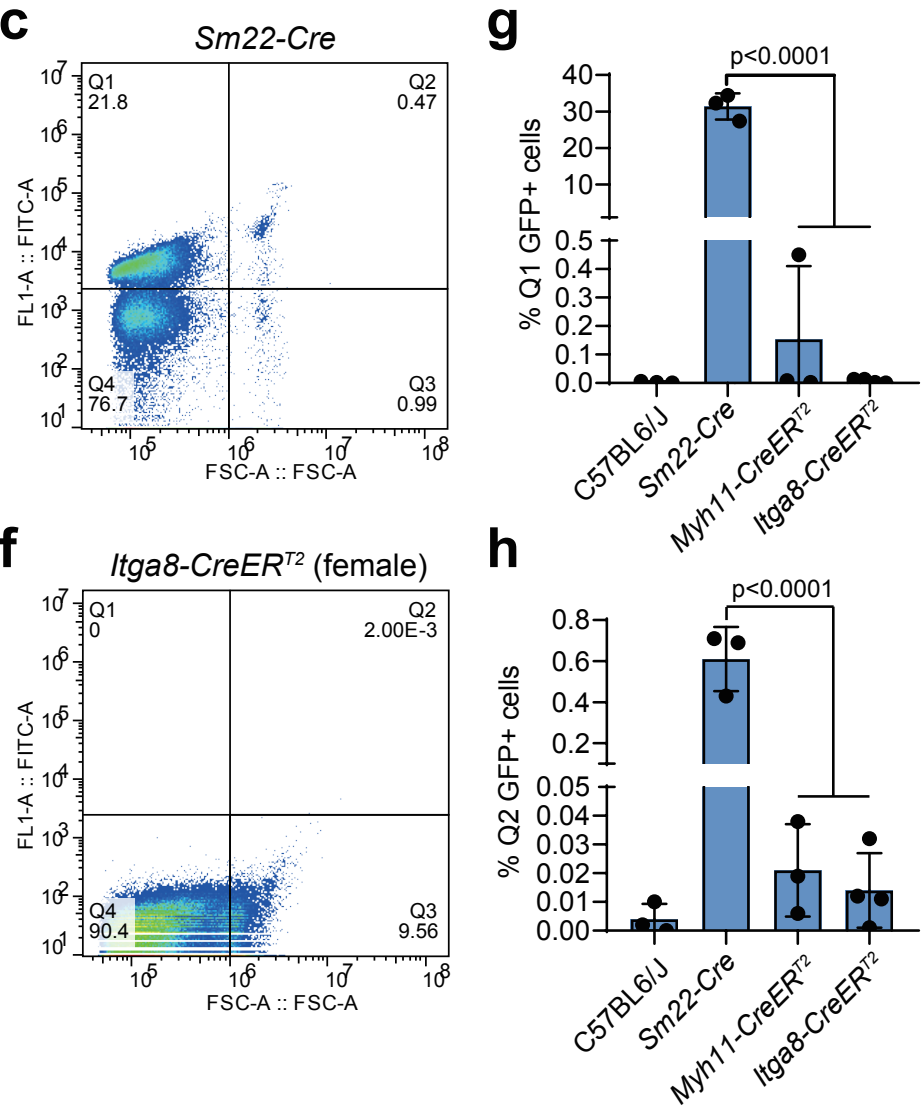
a

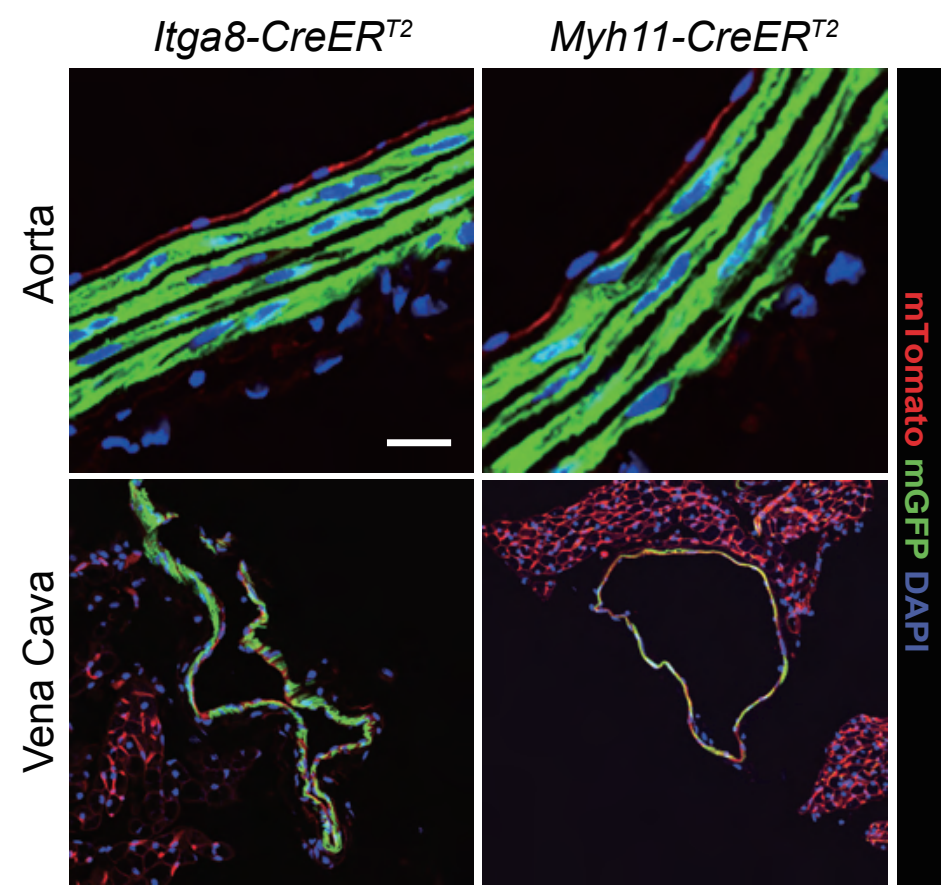

b
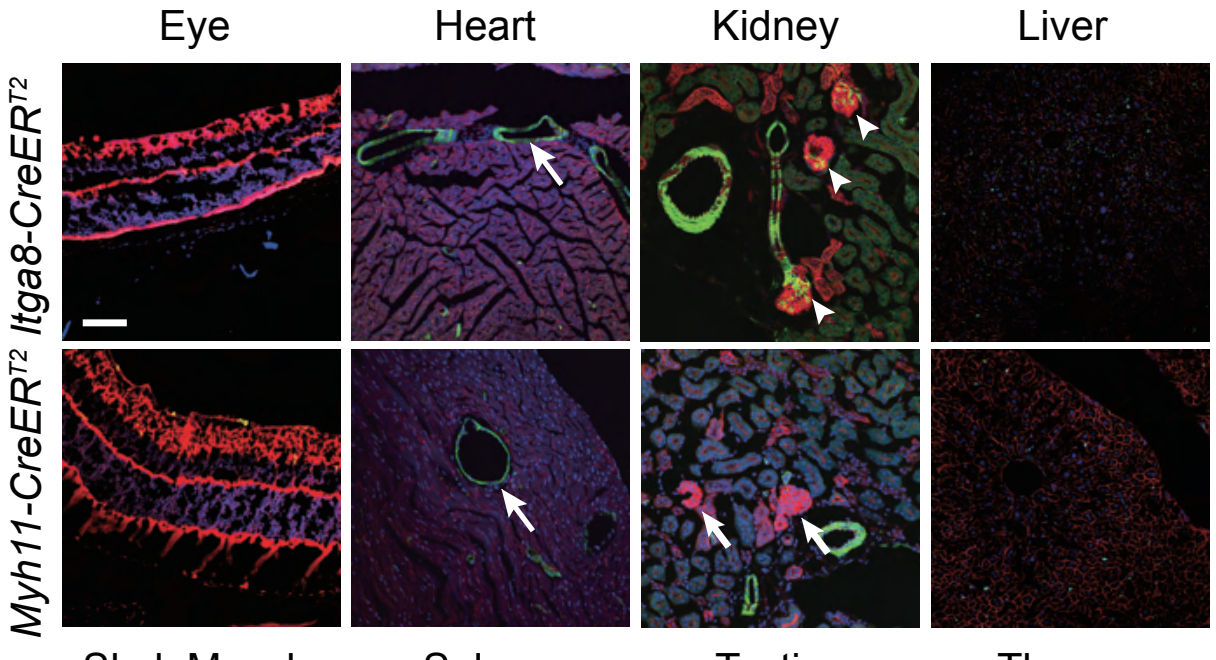

Pancreas
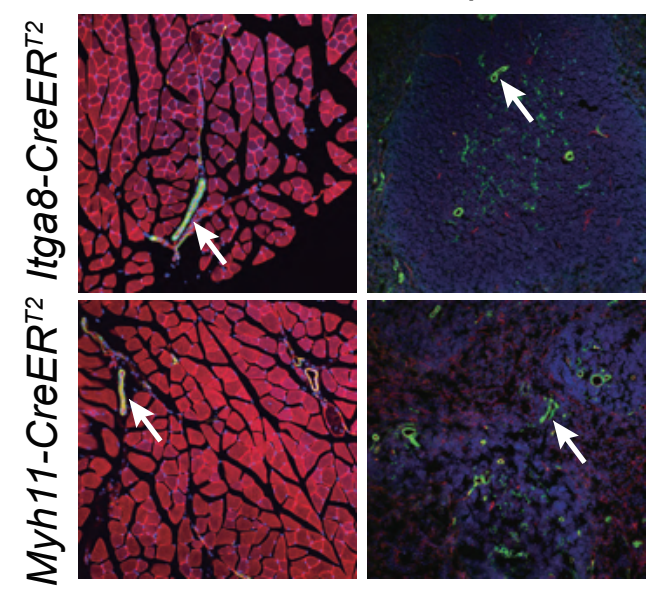

Testis

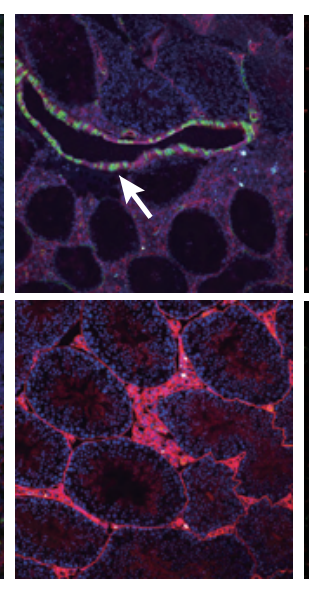

Thymus

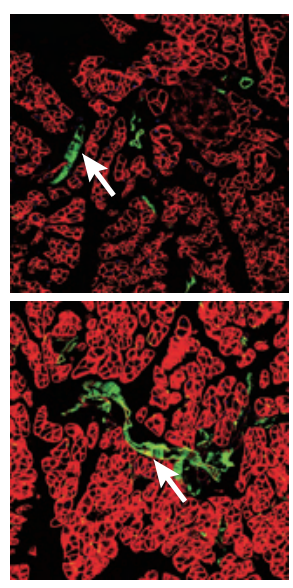

Ovary
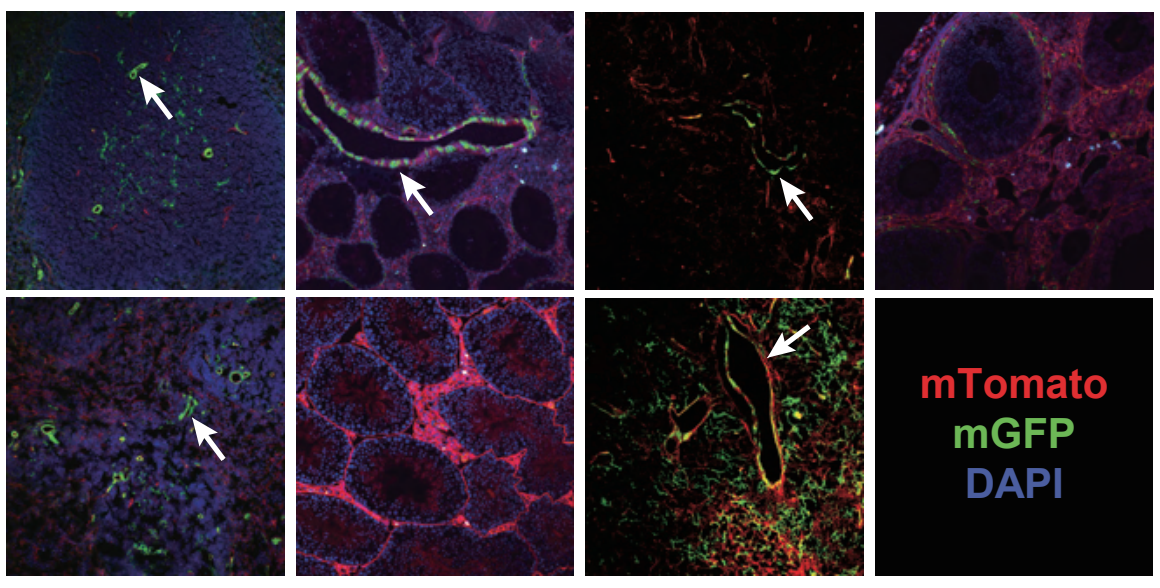
Itga8-CreER
MYh11-iCreER
Itga8-CreER MYh11-iCreER ${ }^{T 2}$

Itga8-CreER $R^{T 2}$ MYh11-iCreER ${ }^{T 2}$

Itga8-CreER ${ }^{T 2}$ MYh11-iCreER ${ }^{T 2}$

Itga 8-CreER ${ }^{T 2}$ MYh11-iCreER ${ }^{T 2}$

Itga 8-CreER ${ }^{T 2}$ MYh11-iCreER ${ }^{T 2}$

Itga8-CreER $R^{T 2}$ MYh11-iCreER ${ }^{T 2}$

Itga8-CreERT2 MYh11-iCreER ${ }^{T 2}$

Itga 8-CreER ${ }^{T 2}$ MYh11-iCreER ${ }^{T 2}$

Itga8-CreER $R^{T 2}$ MYh11-iCreER ${ }^{T 2}$

Itga 8-CreER $R^{T 2}$ MYh11-iCreER ${ }^{T 2}$

Itga 8-CreER ${ }^{T 2}$ MYh11-iCreER ${ }^{T 2}$

Itga8-CreER ${ }^{T 2}$ MYh11-iCreER ${ }^{T 2}$

Itga8-CreER $R^{T 2}$ MYh11-iCreER ${ }^{T 2}$

Itga 8-CreER ${ }^{T 2}$ MYh11-iCreER ${ }^{T 2}$

Itga 8-CreER ${ }^{T 2}$ MYh11-iCreER ${ }^{T 2}$

Itga 8-CreER ${ }^{T 2}$ MYh11-iCreER ${ }^{T 2}$

Itga 8-CreER ${ }^{T 2}$ MYh11-iCreER ${ }^{T 2}$

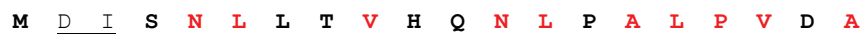
ATG-----TCCAATTTACTGACCGTACACCAAAATTTGCCTGCATTGCCGGTCGATGCA ATGGATATCTCCAACCTGCTGACTGTGCACCAAAACCTGCCTGCCCTCCCTGTGGATGCC

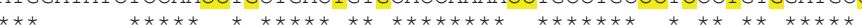

$\begin{array}{llllllllllllllllllll}T & S & D & E & V & R & K & N & L & M & D & M & F & R & D & R & O & A & F & S\end{array}$ ACGAGTGATGAGGTTCGCAAGAACCTGATGGACATGTTCAGGGATCGCCAGGCGTTTTCT ACCTCTGATGAAGTCAGGAAGAACCTGATGGACATGTTCAGGGACAGGCAGGCCTTCTCT

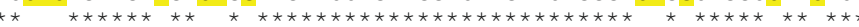

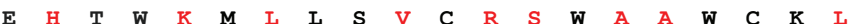
GAGCATACCTGGAAAATGCTTCTGTCCGTTTGCCGGTCGTGGGCGGCATGGTGCAAGTTG GAACACACCTGGAAGATGCTCCTGTCTGTGTGCAGATCCTGGGCTGCCTGGTGCAAGCTG

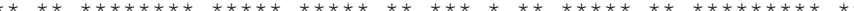

$\begin{array}{lllllllllllllllllllll}\mathbf{N} & \mathbf{N} & \mathbf{R} & \mathbf{K} & \mathbf{W} & \mathbf{F} & \mathrm{P} & \mathbf{A} & \mathbf{E} & \mathbf{P} & \mathrm{E} & \mathrm{D} & \mathrm{V} & \mathrm{R} & \mathrm{D} & \mathrm{Y} & \mathrm{L} & \mathrm{L} & \mathrm{Y} & \mathrm{L}\end{array}$ AATAACCGGAAATGGTTTCCCGCAGAACCTGAAGATGTTCGCGATTATCTTCTATATCTT AACAACAGGAAATGGTTCCCTGCTGAACCTGAGGATGTGAGGGACTACCTCCTGTACCTG **

$\begin{array}{llllllllllllllllllll} & \mathrm{O} & \mathrm{R} & \mathrm{G} & \mathrm{I} & \mathrm{A} & \mathrm{V} & \mathrm{K} & \mathrm{T} & \mathrm{I} & \mathrm{O} & \mathrm{O} & \mathrm{H} & \mathrm{I} & \mathrm{G} & \mathrm{O} & \mathrm{L} & \mathbf{N} & \mathrm{M} & \mathrm{L}\end{array}$ CAGGCGCGCGGTCTGGCAGTAAAAACTATCCAGCAACATTTGGGCCAGCTAAACATGCTT CAAGCCAGAGGCCTGGCTGTGAAGACCATCCAACAGCACCTGGGCCAGCTCAACATGCTG

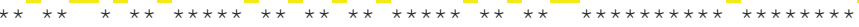

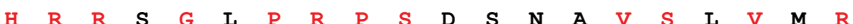
CATCGTCGGTCCGGGCTGCCACGACCAAGTGACAGCAATGCTGTTTCACTGGTTATGCGG CACAGGAGATCTGGCCTGCCTCGCCCTTCTGACTCCAATGCTGTGTCCCTGGTGATGAGG

$\begin{array}{llllllllllllllllllllll}R & I & R & K & E & N & V & D & A & G & E & R & A & K & Q & A & L & A & F & E\end{array}$ CGCATCCGAAAAGAAAACGTTGATGCCGGTGAACGTGCAAAACAGGCTCTAGCGTTCGAA AGAATCAGAAAGGAGAATGTGGATGCTGGGGAGAGAGCCAAGCAGGCCCTGGCCTTTGAA

R T D D O V R S I M E N S D R C O D I CGCACTGATTTCGACCAGGTTCGTTCACTCATGGAAAATAGCGACCGCTGCCAGGATATA CGCACTGACTTTGACCAAGTCAGATCCCTGATGGAGAACTCTGACAGATGCCAGGACATC

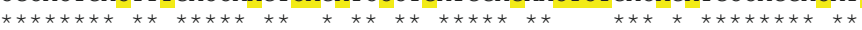

$\begin{array}{llllllllllllllllllll}R & N & \text { L } & \text { A } & \text { F } & \text { L } & G & \text { I } & \text { A } & \text { Y } & \text { N } & \text { T } & \text { L } & \text { L } & \text { R } & \text { I } & \mathbf{A} & \mathbf{E} & \text { I } & \mathbf{A}\end{array}$ CGTAATCTGGCATTTCTGGGGATTGCTTATAACACCCTGTTACGTATAGCCGAAATTGCC AGGAACCTGGCCTTCCTGGGCATTGCCTACAACACCCTGCTGCGCATTGCCGAAATTGCC

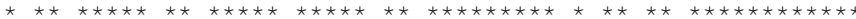

$\begin{array}{llllllllllllllllllllllll}R & I & R & V & K & D & I & S & R & T & D & G & G & R & M & I & I & H & I & G\end{array}$ AGGATCAGGGTTAAAGACATCTCACGTACTGACGGTGGGAGAATGTTAATCCATATTGGC AGAATCAGAGTGAAGGACATCTCCCGCACCGATGGTGGGAGAATGCTGATCCACATTGGC

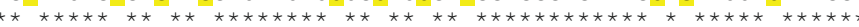

$\begin{array}{llllllllllllllllllll}R & \text { T } & \text { K } & \text { T } & \text { L } & \text { V } & \text { S } & \text { T } & \text { A } & \text { G } & \text { V } & \text { E } & \text { K } & \text { A } & \text { L } & \text { S } & \text { L } & \text { G } & \text { V } & \text { T }\end{array}$ AGAACGAAAACGCTGGTTAGCACCGCAGGTGTAGAGAAGGCACTTAGCCTGGGGGTAACT AGGACCAAGACCCTGGTGTCCACAGCTGGTGTGGAGAAGGCCCTGTCCCTGGGGGTTACC

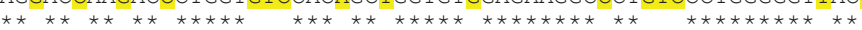

$\begin{array}{llllllllllllllllllll}\text { K } & \text { L } & \text { V } & \text { E } & \text { R } & \text { W } & \text { I } & \text { S } & \text { V } & \text { S } & \text { G } & \text { V } & \text { A } & \text { D } & \text { D } & \text { P } & \text { N } & \text { N } & \text { Y } & \text { L }\end{array}$ AAACTGGTCGAGCGATGGATTTCCGTCTCTGGTGTAGCTGATGATCCGAATAACTACCTG AAGCTGGTGGAGAGATGGATCTCTGTGTCTGGTGTGGCTGATGACCCCAACAACTACCTG

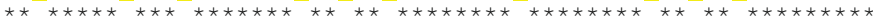

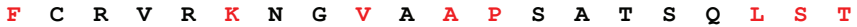
TTTTGCCGGGTCAGAAAAAATGGTGTTGCCGCGCCATCTGCCACCAGCCAGCTATCAACT TTCTGCCGGGTCAGAAAGAATGGTGTGGCTGCCCCTTCTGCCACCTCCCAACTGTCCACC

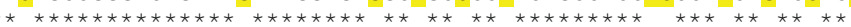

$\begin{array}{llllllllllllllllllll}R & A & \text { L } & E & G & I & F & E & A & \text { T } & \text { H } & \text { R } & \text { L } & \text { I } & Y & G & \text { A } & K & \text { D } & \text { D }\end{array}$ CGCGCCCTGGAAGGGATTTTTGAAGCAACTCATCGgTTGATTTACGGCGCTAAGGATGAC CGGGCCCTGGAAGGGATCTTTGAGGCCACCCACCGCCTGATCTATGGTGCCAAGGATGAC

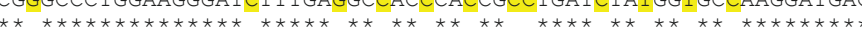

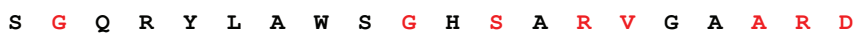
TCTGGTCAGAGATACCTGGCCTGGTCTGGACACAGTGCCCGTGTCGGAGCCGCGCGAGAT TCTGGGCAGAGATACCTGGCCTGGTCTGGCCACTCTGCCAGAGTGGGTGCTGCCAGGGAC

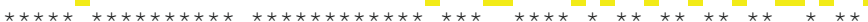

$\begin{array}{llllllllllllllllllll}M & A & R & A & G & V & S & I & \text { P } & \text { E } & \text { I } & \text { M } & \text { Q } & \text { A } & \text { G } & \text { G } & \text { W } & \text { T } & \text { N } & \text { V }\end{array}$ ATGGCCCGCGCTGGAGTTTCAATACCGGAGATCATGCAAGCTGGTGGCTGGACCAATGTA ATGGCCAGGGCTGGTGTGTCCATCCCTGAAATCATGCAGGCTGGTGGCTGGACCAATGTG

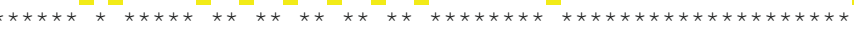

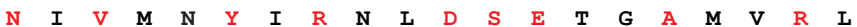
AATATTGTCATGAACTATATCCGTAACCTGGATAGTGAAACAGGGGCAATGGTGCGCCTG AACATTGTGATGAACTACATCAGAAACCTGGACTCTGAGACTGGGGCCATGGTGAGGCTG

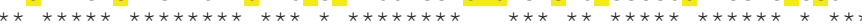


a

Itga8-CreER ${ }^{T 2} m T / m G$ Popliteal Artery 20x Live
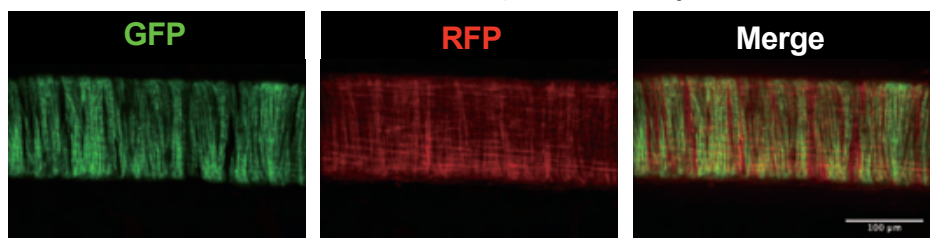

b

Myh11-CreER ${ }^{T 2} \mathrm{mT} / m$ G Popliteal Artery 20x Live
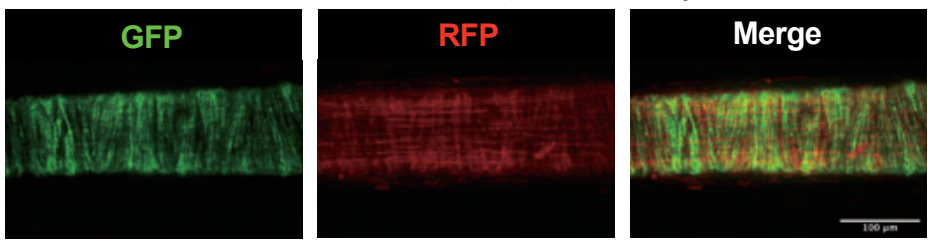

C

Itga8-CreER $R^{T 2} \mathrm{mT} / \mathrm{mG}$ Popliteal Vein 20x Live
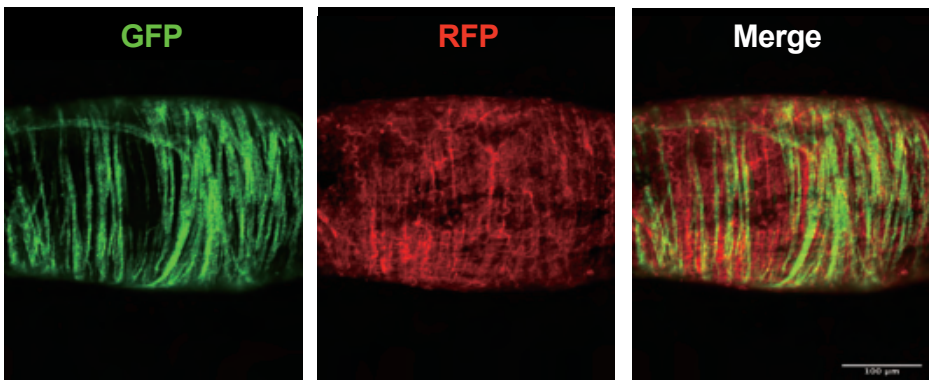

d

Myh11-CreER ${ }^{T 2} m T / m G$ Popliteal Vein 20x Live

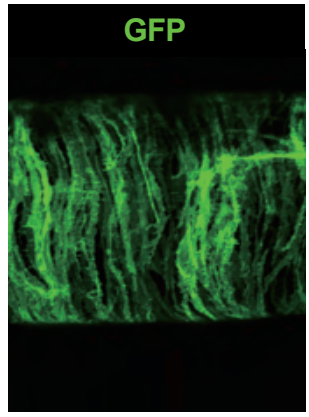

e

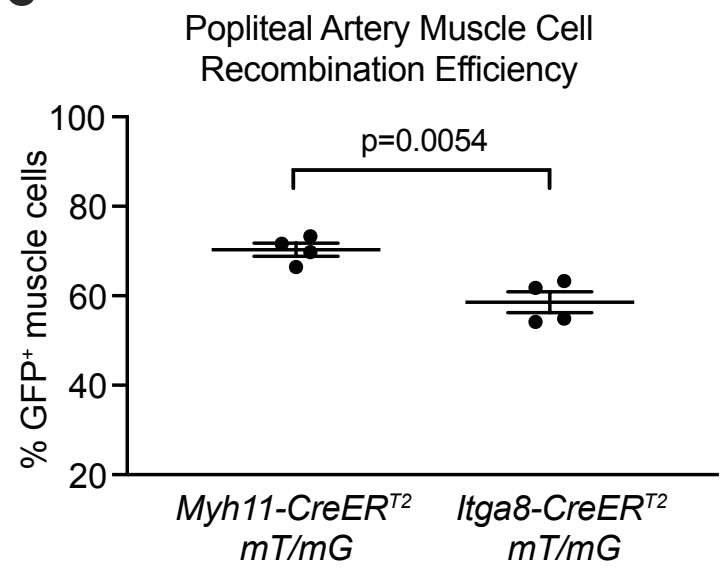

$\mathbf{f}$

Popliteal Vein Muscle Recombination Efficiency

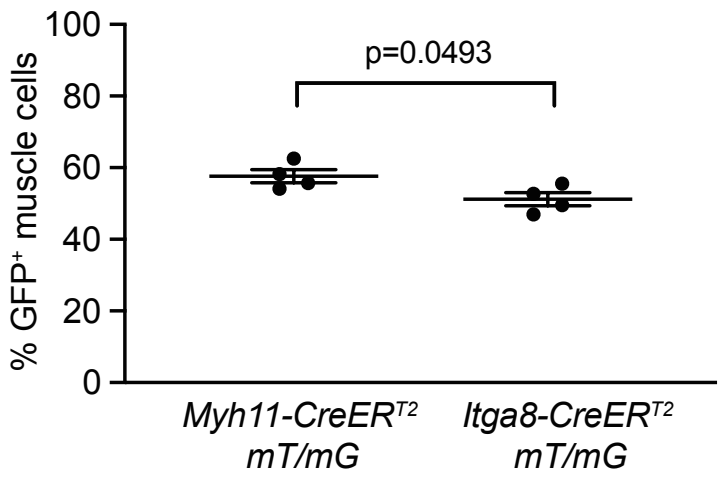


a

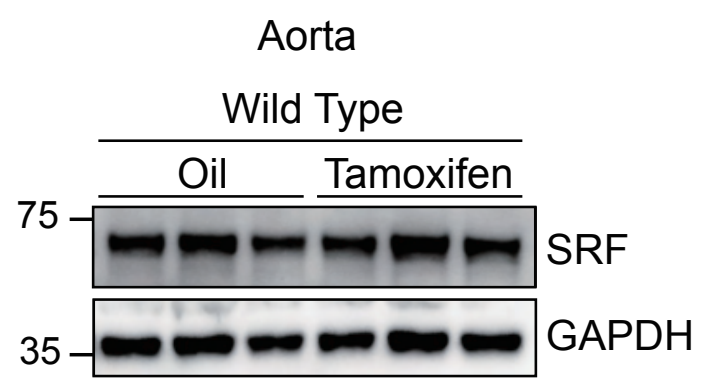

b

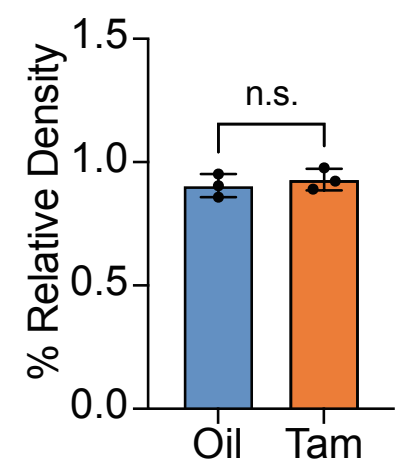

C

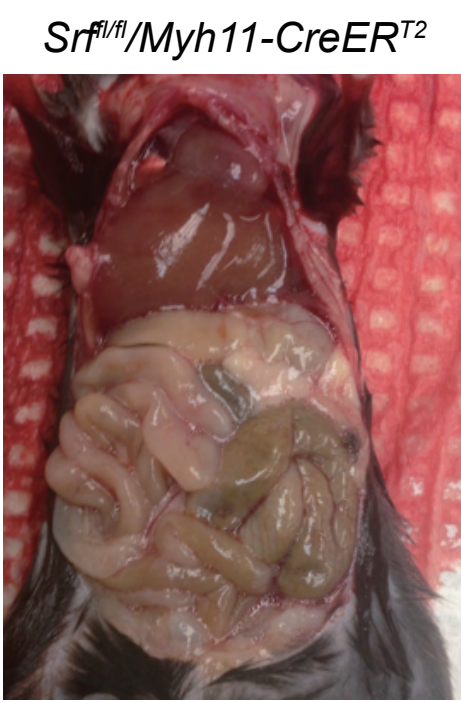
Srf $f^{f / f} / M y h 11-C r e E R^{T 2}$ Srff(If//Itga8-CreER ${ }^{T 2}$

d
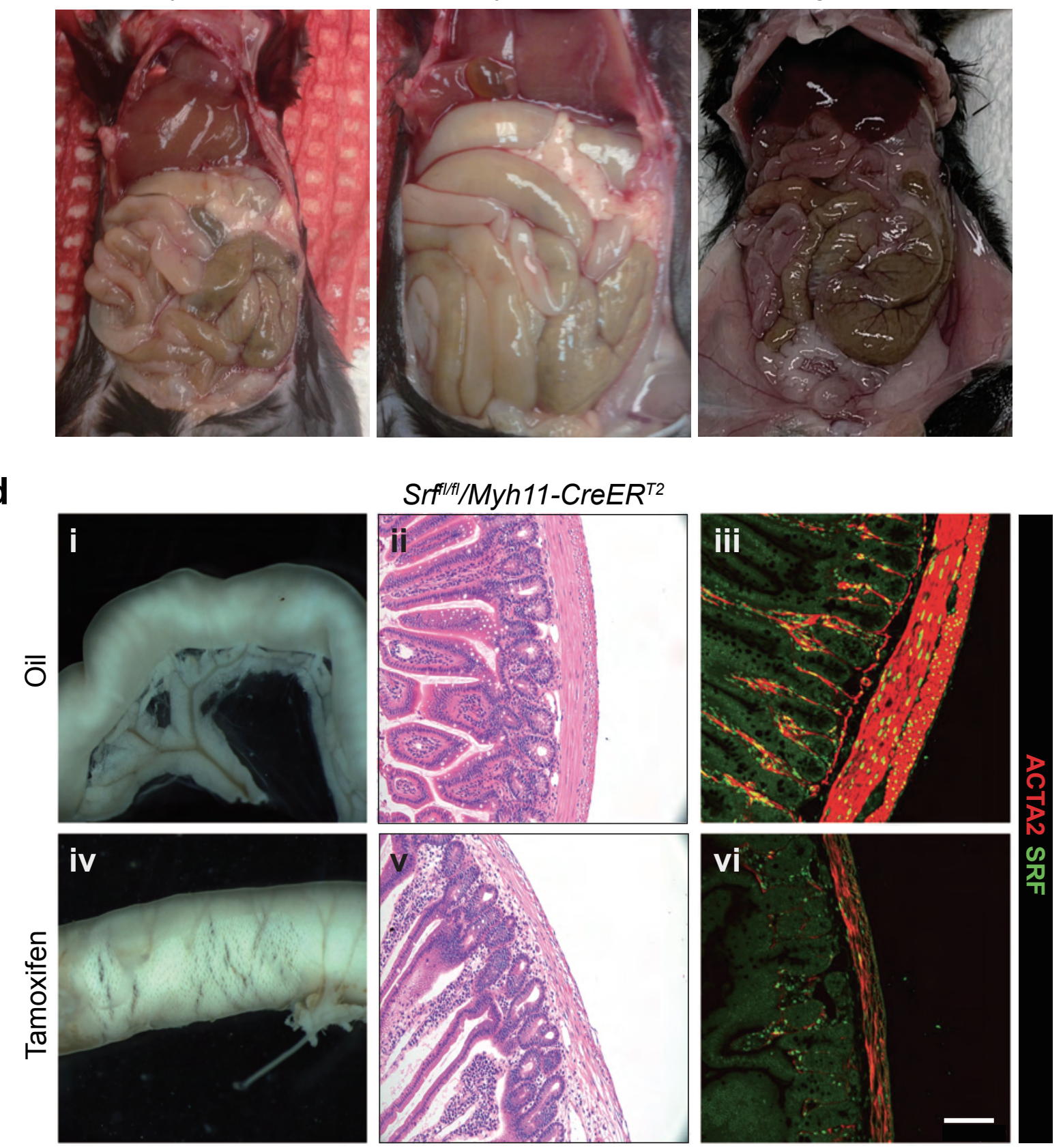

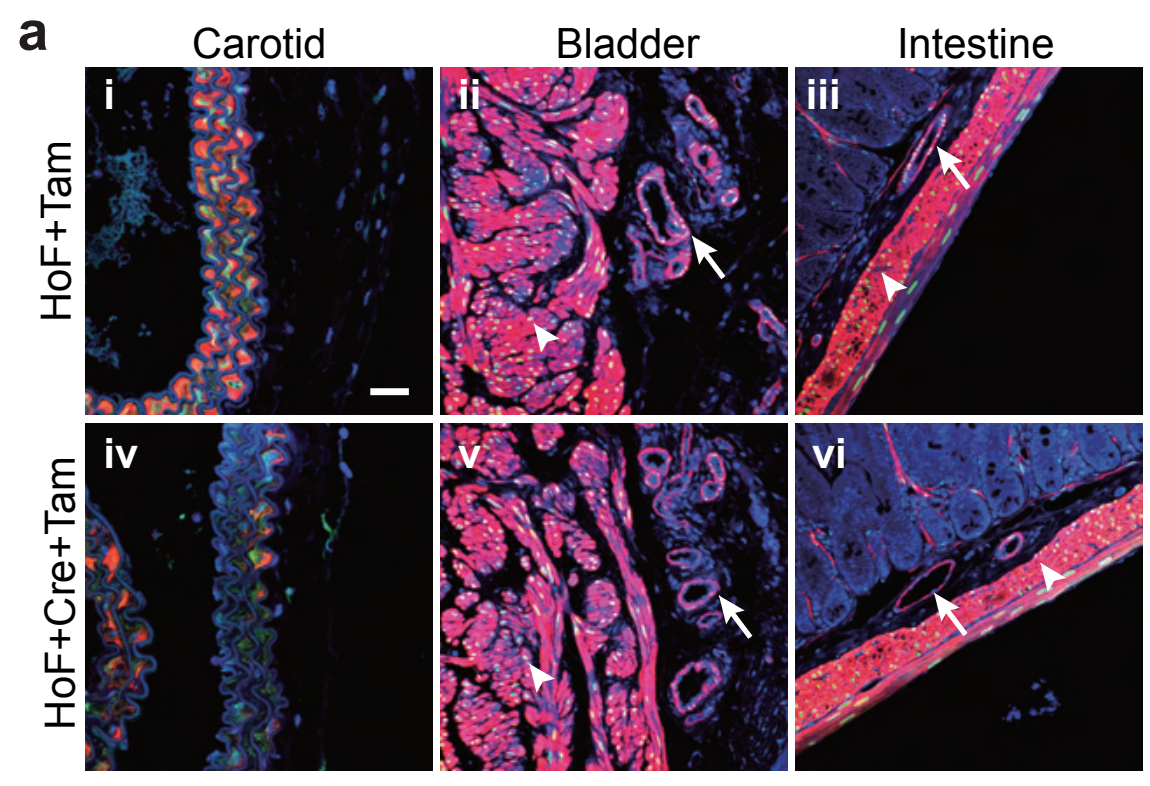

d

b

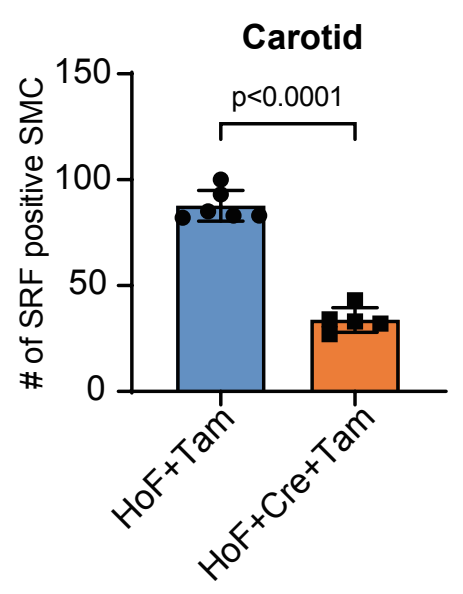

C

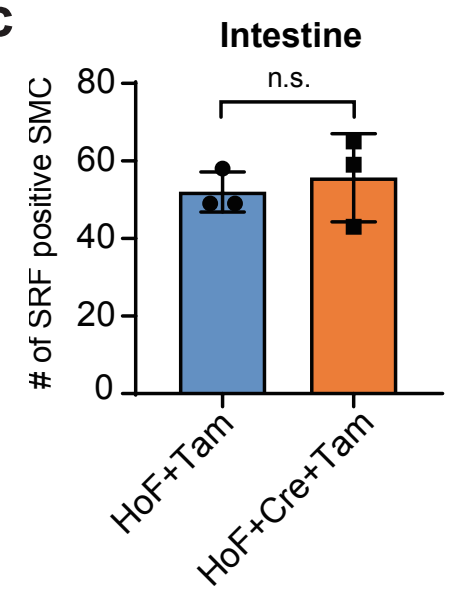

f

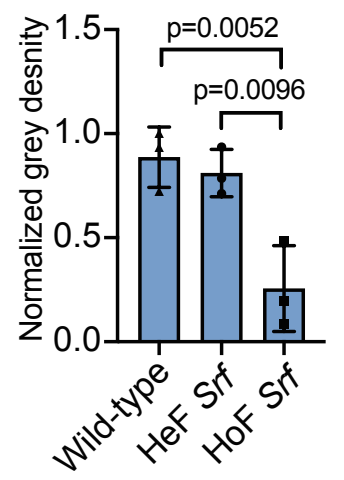

Aorta
Aorta

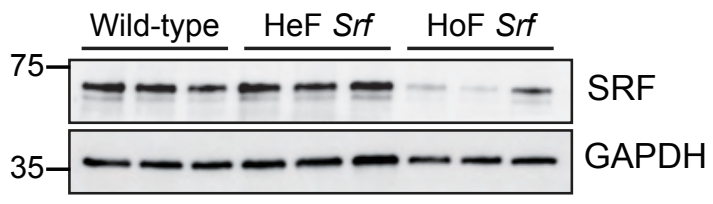

Bladder

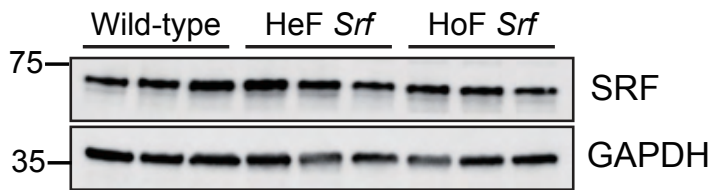

g

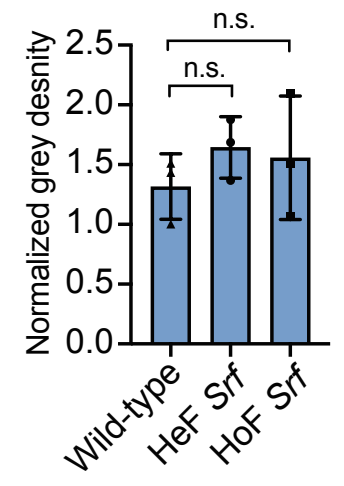




\section{Supplementary Fig. 11}
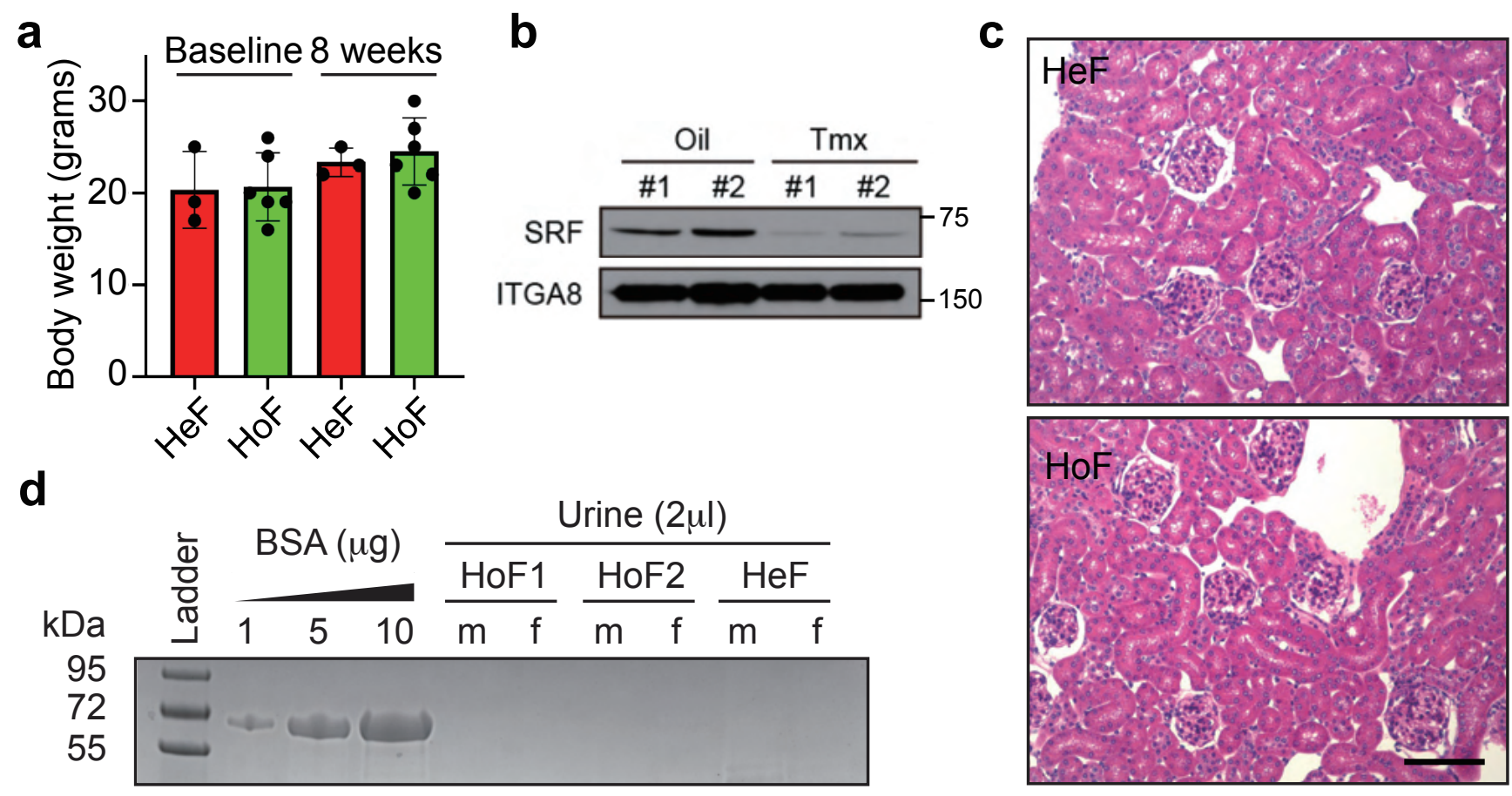